\title{
Stereoselective Synthesis of Ribofuranoid exo-Glycals by One-Pot Julia Olefination Using
}

\section{Ribofuranosyl Sulfones}

\author{
Natsuhisa Oka, ${ }^{1,2,3, *}$ Ayumi Mori, ${ }^{1}$ Kanna Suzuki, ${ }^{1}$ and Kaori Ando ${ }^{1, *}$ \\ ${ }^{1}$ Department of Chemistry and Biomolecular Science, Faculty of Engineering, Gifu University \\ ${ }^{2}$ Center for Highly Advanced Integration of Nano and Life Sciences, Gifu University (G-CHAIN) \\ 1-1 Yanagido, Gifu 501-1193, Japan \\ ${ }^{3}$ Institute for Glyco-core Research (iGCORE), Gifu University, Tokai National Higher Education and \\ Research System \\ Furo-cho, Chikusa-ku, Nagoya 464-8601, Japan \\ oka@gifu-u.ac.jp,ando@gifu-u.ac.jp
}

\section{Supporting information}

\section{Contents:}

1. X-ray structure analysis. Crystal data, structure refinement results, bond lengths, and bond angles of 4a (S2-S4)

2. Computational details. The reaction mechanism of the one-pot Julia olefination of $\beta$-ribofuranosyl sulfone with PhCHO. (S4-S5)

3. The xyz coordinates, the Gibbs free energies (hartree), and the number of imaginary frequencies for the calculated structures (E)-A, (Z)-A, $\alpha E$-TS1, $\alpha E$-TS2, $\alpha E$-TS3, $\alpha Z$-TS1, $\alpha Z$-TS2, $\alpha Z$-TS3, $\beta E$-TS1, $\beta E$-TS2, $\beta E$-TS3, $\beta Z$-TS1, $\beta Z$-TS2, and $\beta Z$-TS3. (S6-S12)

4. References for Supporting information (S13)

5. NMR spectra of 3a, 4a-d, 5a-m, (Z)-5a, $(Z)-\mathbf{5 c}, \mathbf{7}, \beta-\mathbf{4 a}, \beta-3 \mathbf{a}^{\prime}, \beta-\mathbf{4} \mathbf{a}^{\prime}, \mathbf{1 0}, \mathbf{1 1}$, epi-11, 17-24 (S14-S87) 


\section{X-ray structure analysis}

The measurement of $\alpha-\mathbf{4 a}$ was carried out on a Rigaku/MSC Mercury CCD diffractometer with graphite-monochromated Mo-Ka radiation $(\lambda=0.71069 \AA)$. Reflection data were collected at $193 \mathrm{~K}$ using a Rigaku XR-TCS-2-050 temperature controller. The structure was solved by direct methods (Sir2014) ${ }^{1}$ and refined by full-matrix least-squares procedures (SHELXL-2014) ${ }^{2}$ using the Yadokari-XG 2009. ${ }^{3}$ The X-ray quality crystals were obtained by recrystallization from $\mathrm{CH}_{2} \mathrm{Cl}_{2}-$ hexane $(7: 20, \mathrm{v} / \mathrm{v})$. The crystal was cut from a grown crystal and attached to the tip of a MiTeGen MicroMount ${ }^{\mathrm{TM}}$. The crystal data and structure refinement results are summarized as the supplementary material. Crystallographic data for $\alpha-4 a$ (CCDC 2009571) can be obtained free of charge from The Cambridge Crystallographic Data Centre via www.ccdc.cam.ac.uk/data request/cif.

(a)

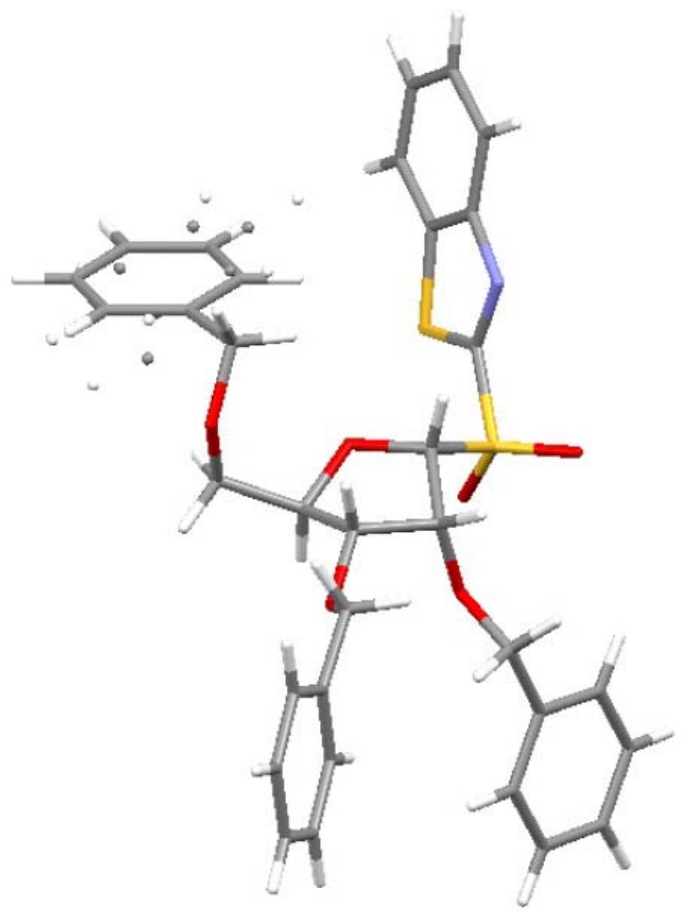

(b)

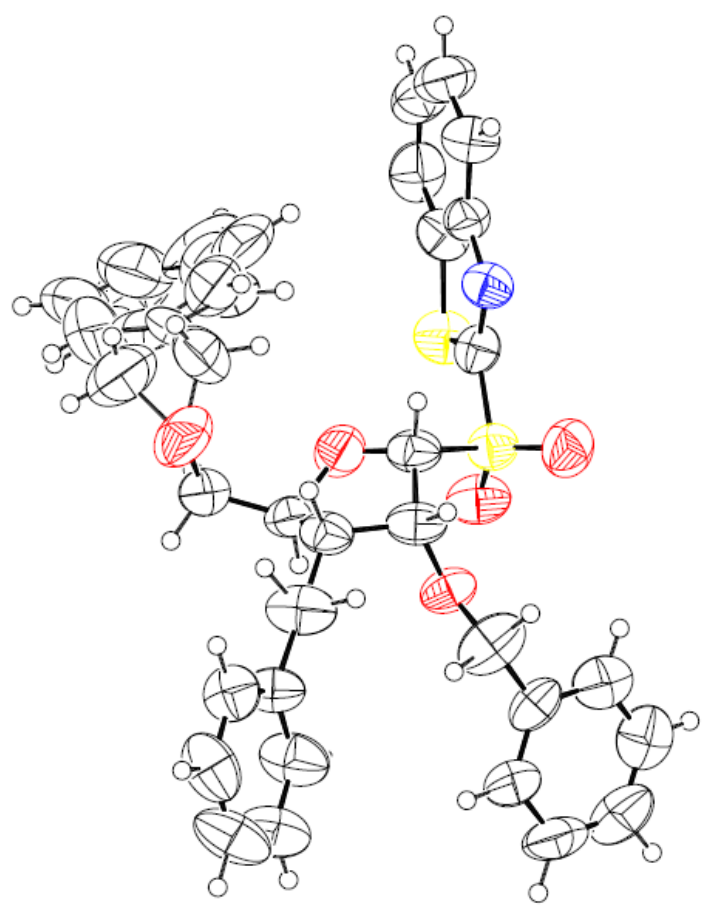

(a) X-ray crystal structure of 4a. (b) ORTEP drawing of 4a (thermal ellipsoids drawn at 50\% probability level).

Table S1. Crystal data and structure refinement of $4 \mathbf{a}$.

\begin{tabular}{ll}
\hline Identification code & BTsulfone \\
Chemical formula & $\mathrm{C}_{33} \mathrm{H}_{31} \mathrm{NO}_{6} \mathrm{~S}_{2}$ \\
$M_{\mathrm{r}}$ & 601.71 \\
Crystal system, space group & Monoclinic, $P 2_{1}$ \\
Temperature (K) & 193 \\
$a, b, c(\AA)$ & $5.9073(5), 18.4043(15), 14.0127(12)$ \\
$\beta\left(^{\circ}\right)$ & $95.268(8)$ \\
$V\left(\AA^{3}\right)$ & $1517.0(2)$
\end{tabular}


Z

Radiation type

$\mu\left(\mathrm{mm}^{-1}\right)$

Crystal size (mm)

Data collection

Diffractometer

Absorption correction

$T_{\min }, T_{\max }$

No. of measured, independent and

observed $[I>2 \sigma(I)]$ reflections

$R_{\text {int }}$

$(\sin \theta / \lambda)_{\max }\left(\AA^{-1}\right)$

Refinement

$R\left[F^{2}>2 \sigma\left(F^{2}\right)\right], w R\left(F^{2}\right), S$

No. of reflections

No. of parameters

No. of restraints

$\mathrm{H}$-atom treatment

$\left.\Delta\rangle_{\max }, \Delta\right\rangle_{\min }\left(\mathrm{e} \AA^{-3}\right)$

Absolute structure

Absolute structure parameter
2

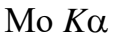

0.22

$0.20 \times 0.06 \times 0.03$

Rigaku Mercury CCD ( $2 \times 2$ bin mode)

Numerical

$0.963,0.997$

13858, 6096, 2515

0.069

0.650

$0.063, \quad 0.214, \quad 1.01$

6096

419

116

$\mathrm{H}$-atom parameters constrained

$0.16,-0.24$

Flack x determined using 674 quotients $[(\mathrm{I}+)-(\mathrm{I}-)] /[(\mathrm{I}+)+(\mathrm{I}-)] \quad$ (Parsons,

Flack and Wagner, Acta Cryst. B69 (2013) 249-259).

$0.07(9)$

Computer programs: CrystalClear-SM Expert 2.0 r16 (Rigaku, 2014), SHELXL2014/7 (Sheldrick, 2014).

Table S2. Selected geometric parameters $\left(\AA,{ }^{\circ}\right)$

\begin{tabular}{llll}
\hline $\mathrm{S} 1-\mathrm{O} 1$ & $1.430(7)$ & $\mathrm{C} 9-\mathrm{C} 10$ & $1.529(14)$ \\
$\mathrm{S} 1-\mathrm{O} 2$ & $1.436(7)$ & $\mathrm{C} 10-\mathrm{O} 5$ & $1.406(10)$ \\
$\mathrm{S} 1-\mathrm{C} 1$ & $\mathrm{C} 10-\mathrm{C} 11$ & $1.523(13)$ \\
$\mathrm{S} 1-\mathrm{C} 8$ & $1.776(10)$ & $\mathrm{C} 11-\mathrm{O} 3$ & $1.453(11)$ \\
$\mathrm{C} 8-\mathrm{C} 9$ & $1.790(10)$ & $\mathrm{C} 11-\mathrm{C} 12$ & $1.499(13)$ \\
$\mathrm{C} 9-\mathrm{O} 6$ & $1.528(13)$ & $\mathrm{C} 12-\mathrm{O} 4$ & $1.378(13)$ \\
$\mathrm{C} 8-\mathrm{O} 3$ & $1.423(10)$ & & \\
$\mathrm{O} 1-\mathrm{S} 1-\mathrm{O} 2$ & $1.404(12)$ & $\mathrm{O}-\mathrm{C} 9-\mathrm{C} 10$ & $111.9(8)$ \\
$\mathrm{O} 1-\mathrm{S} 1-\mathrm{C} 1$ & $121.1(5)$ & $\mathrm{O} 8-\mathrm{C} 9-\mathrm{C} 10-\mathrm{C} 11$ & $99.9(8)$ \\
$\mathrm{O} 2-\mathrm{S} 1-\mathrm{C} 1$ & $104.8(4)$ & $\mathrm{O} 5-\mathrm{C} 10-\mathrm{C} 9$ & $110.6(7)$ \\
$\mathrm{O} 1-\mathrm{S} 1-\mathrm{C} 8$ & $108.4(4)$ & $\mathrm{C} 11-\mathrm{C} 10-\mathrm{C} 9$ & $116.3(8)$ \\
$\mathrm{O} 2-\mathrm{S} 1-\mathrm{C} 8$ & $112.6(5)$ & $\mathrm{O} 3-\mathrm{C} 11-\mathrm{C} 12$ & $101.5(8)$ \\
$\mathrm{C} 1-\mathrm{S} 1-\mathrm{C} 8$ & $107.7(5)$ & $\mathrm{O} 3-\mathrm{C} 11-\mathrm{C} 10$ & $109.1(8)$ \\
$\mathrm{O} 3-\mathrm{C} 8-\mathrm{C} 9$ & $100.1(4)$ & $\mathrm{C} 12-\mathrm{C} 11-\mathrm{C} 10$ & $102.9(8)$ \\
$\mathrm{O} 3-\mathrm{C} 8-\mathrm{S} 1$ & $107.8(8)$ & & $116.9(10)$
\end{tabular}




$\begin{array}{llll}\mathrm{C} 9-\mathrm{C} 8-\mathrm{S} 1 & 115.1(7) & \mathrm{C} 8-\mathrm{O} 3-\mathrm{C} 11 & 109.7(7) \\ \mathrm{O} 6-\mathrm{C} 9-\mathrm{C} 8 & 109.4(8) & \mathrm{O} 4-\mathrm{C} 12-\mathrm{C} 11 & 114.3(10) \\ \mathrm{O} 1-\mathrm{S} 1-\mathrm{C} 1-\mathrm{N} 1 & -177.6(7) & \mathrm{S} 1-\mathrm{C} 8-\mathrm{C} 9-\mathrm{C} 10 & -149.8(7) \\ \mathrm{O} 2-\mathrm{S} 1-\mathrm{C} 1-\mathrm{N} 1 & -47.0(9) & \mathrm{O} 6-\mathrm{C} 9-\mathrm{C} 10-\mathrm{O} 5 & 44.7(11) \\ \mathrm{C} 8-\mathrm{S} 1-\mathrm{C} 1-\mathrm{N} 1 & 65.6(8) & \mathrm{C} 8-\mathrm{C} 9-\mathrm{C} 10-\mathrm{O} 5 & 160.4(8) \\ \mathrm{O} 1-\mathrm{S} 1-\mathrm{C} 1-\mathrm{S} 2 & 5.0(7) & \mathrm{O}-\mathrm{C} 9-\mathrm{C} 10-\mathrm{C} 11 & -75.3(9) \\ \mathrm{O} 2-\mathrm{S} 1-\mathrm{C} 1-\mathrm{S} 2 & 135.6(5) & \mathrm{C} 8-\mathrm{C} 9-\mathrm{C} 10-\mathrm{C} 11 & 40.4(9) \\ \mathrm{C} 8-\mathrm{S} 1-\mathrm{C} 1-\mathrm{S} 2 & -111.8(6) & \mathrm{O} 5-\mathrm{C} 10-\mathrm{C} 11-\mathrm{O} 3 & -163.6(7) \\ \mathrm{C} 6-\mathrm{C} 7-\mathrm{N} 1-\mathrm{C} 1 & -179.7(9) & \mathrm{C} 9-\mathrm{C} 10-\mathrm{C} 11-\mathrm{O} 3 & -39.6(9) \\ \mathrm{O} 1-\mathrm{S} 1-\mathrm{C} 8-\mathrm{O} 3 & -43.2(8) & \mathrm{C} 9-\mathrm{C} 10-\mathrm{C} 11-\mathrm{C} 12 & -159.2(9) \\ \mathrm{O} 2-\mathrm{S} 1-\mathrm{C} 8-\mathrm{O} 3 & -179.2(6) & \mathrm{C} 9-\mathrm{C} 8-\mathrm{O} 3-\mathrm{C} 11 & 3.5(10) \\ \mathrm{C} 1-\mathrm{S} 1-\mathrm{C} 8-\mathrm{O} 3 & 67.7(7) & \mathrm{S} 1-\mathrm{C} 8-\mathrm{O} 3-\mathrm{C} 11 & 129.0(7) \\ \mathrm{O} 1-\mathrm{S} 1-\mathrm{C} 8-\mathrm{C} 9 & 78.1(8) & \mathrm{C} 12-\mathrm{C} 11-\mathrm{O} 3-\mathrm{C} 8 & 147.7(9) \\ \mathrm{O} 2-\mathrm{S} 1-\mathrm{C} 8-\mathrm{C} 9 & -58.0(8) & \mathrm{C} 10-\mathrm{C} 11-\mathrm{O} 3-\mathrm{C} 8 & 22.8(10) \\ \mathrm{C} 1-\mathrm{S} 1-\mathrm{C} 8-\mathrm{C} 9 & -171.1(7) & \mathrm{O} 3-\mathrm{C} 11-\mathrm{C} 12-\mathrm{O} 4 & -72.3(12) \\ \mathrm{O} 3-\mathrm{C} 8-\mathrm{C} 9-\mathrm{O} 6 & 89.6(10) & \mathrm{C} 10-\mathrm{C} 11-\mathrm{C} 12-\mathrm{O} 4 & 43.9(13) \\ \mathrm{S} 1-\mathrm{C} 8-\mathrm{C} 9-\mathrm{O} 6 & -32.2(11) & & \\ \mathrm{O} 3-\mathrm{C} 8-\mathrm{C} 9-\mathrm{C} 10 & -27.9(10) & & \end{array}$

\section{Computational details}

All calculations were performed using the Gaussian 03 and 09 programs. ${ }^{4}$ Gibbs free energies are the values at $298.15 \mathrm{~K}$ and $1.00 \mathrm{~atm}$ obtained from frequency calculations. All calculations were performed by the B3LYP hybrid functional together with the $6-31+\mathrm{G}^{*}$ basis set. Vibrational frequency calculations gave only one imaginary frequency for all transition structures and confirmed that those structures are authentic transition structures. Starting from the transition state structures, the reaction paths were followed by the intrinsic reaction coordinate (IRC) analysis. ${ }^{5}$ The structures of the reactants, products, and intermediates were obtained from the optimization of the last structures on both sides of the IRC calculations. Their frequency calculations gave only harmonic frequencies and confirmed that they are minima.
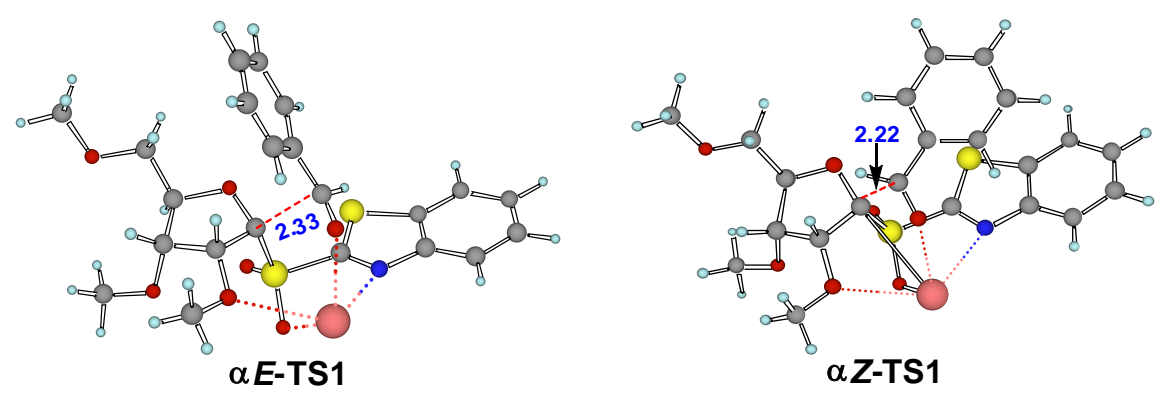

Figure S1. The structures TS1 in Figure 1. 

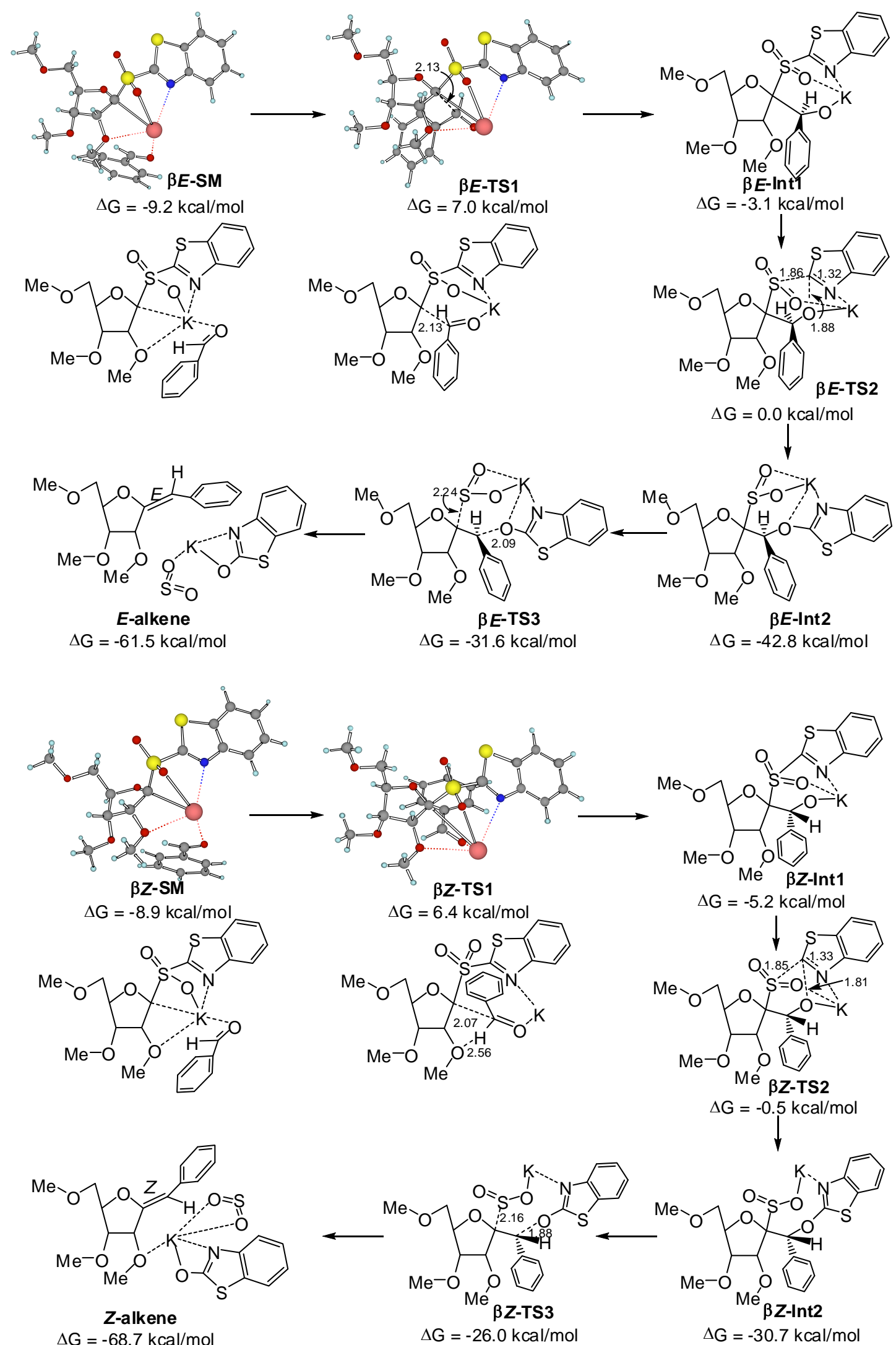

Figure S2. The full reaction path of the reaction of $\beta$-ribofuranosyl sulfone with PhCHO. 
3. Coordinates and the energies (Hartree) of the structures shown in Figure 1-3 and Figure S2.

$\mathrm{G}$ is the Gibbs free energy at $298.15 \mathrm{~K}$ (B3LYP/6-31G* level).

\begin{tabular}{|c|c|c|c|c|c|c|c|c|c|}
\hline \multicolumn{5}{|c|}{$\begin{array}{c}(E)-\mathbf{A}, \mathrm{G}=-884.177570 \text { Hartree } \\
0 \text { imaginary frequencies }\end{array}$} & \multicolumn{5}{|c|}{$\begin{array}{c}(Z)-\mathbf{A}, \mathrm{G}=-884.180672 \text { Hartree } \\
0 \text { imaginary frequencies }\end{array}$} \\
\hline 6 & 0 & 0.001810 & 0.691261 & 0.021011 & 6 & 0 & -0.934937 & 1.194252 & -0.695814 \\
\hline 6 & 0 & -0.318528 & -0.720228 & $3-0.407890$ & 6 & 0 & 0.304846 & 0.407301 & -0.332860 \\
\hline 6 & 0 & 1.474625 & 0.524169 & 0.476938 & 6 & 0 & -2.012535 & 0.580510 & 0.238975 \\
\hline 1 & 0 & -0.648718 & 1.032258 & 0.836956 & 1 & 0 & -1.229683 & 0.954100 & -1.735644 \\
\hline 8 & 0 & -0.126123 & 1.555314 & -1.104019 & 8 & 0 & -0.709481 & 2.571784 & -0.576572 \\
\hline 1 & 0 & 1.470033 & 0.067810 & 1.481703 & 1 & 0 & -3.016100 & 0.585110 & -0.208265 \\
\hline 8 & 0 & 2.152566 & 1.754530 & 0.490965 & 8 & 0 & -2.008672 & 1.300889 & 1.453090 \\
\hline 6 & 0 & 1.988613 & -0.520860 & -0.539166 & 6 & 0 & -1.495477 & -0.861738 & 0.402773 \\
\hline 1 & 0 & 2.254239 & -0.009634 & -1.472950 & 1 & 0 & -1.655347 & -1.216883 & 1.425184 \\
\hline 6 & 0 & 3.118055 & -1.453002 & -0.118282 & 6 & 0 & -2.103686 & -1.866723 & -0.573860 \\
\hline 8 & 0 & 0.846342 & -1.373307 & -0.752005 & 8 & 0 & -0.070055 & -0.810463 & 0.178224 \\
\hline 6 & 0 & -0.269391 & 2.936384 & -0.783991 & 6 & 0 & -1.783874 & 3.370415 & -1.036517 \\
\hline 6 & 0 & 3.225261 & 1.843031 & 1.420141 & 6 & 0 & -3.135192 & 1.043621 & 2.271807 \\
\hline 1 & 0 & 3.145709 & -2.300825 & -0.821215 & 1 & 0 & -1.565710 & -2.824287 & -0.490112 \\
\hline 1 & 0 & 2.914801 & -1.863844 & 0.885628 & 1 & 0 & -1.987222 & -1.514501 & -1.613609 \\
\hline 8 & 0 & 4.343676 & -0.748802 & -0.143471 & 8 & 0 & -3.468449 & -2.013231 & -0.238449 \\
\hline 6 & 0 & 5.451103 & -1.573668 & 0.160335 & 6 & 0 & -4.139938 & -2.949070 & -1.058264 \\
\hline 1 & 0 & -0.456561 & 3.448088 & -1.731745 & 1 & 0 & -1.447194 & 4.408392 & -0.981295 \\
\hline 1 & 0 & 0.631350 & 3.340713 & -0.315889 & 1 & 0 & -2.675624 & 3.251634 & -0.406737 \\
\hline 1 & 0 & -1.130721 & 3.097757 & -0.116896 & 1 & 0 & -2.046661 & 3.133950 & -2.080338 \\
\hline 1 & 0 & 3.605285 & 2.865724 & 1.354099 & 1 & 0 & -3.024645 & 1.668649 & 3.161165 \\
\hline 1 & 0 & 4.025518 & 1.136766 & 1.176421 & 1 & 0 & -3.198043 & -0.009564 & 2.582617 \\
\hline 1 & 0 & 2.875121 & 1.660513 & 2.448300 & 1 & 0 & -4.073280 & 1.306682 & 1.758184 \\
\hline 1 & 0 & 6.344337 & -0.946760 & 0.102973 & 1 & 0 & -5.177779 & -2.988246 & -0.718062 \\
\hline 1 & 0 & 5.545414 & -2.401224 & -0.559895 & 1 & 0 & -3.696026 & -3.953250 & -0.973782 \\
\hline 1 & 0 & 5.375464 & -2.001233 & 1.172719 & 1 & 0 & -4.120302 & -2.648597 & -2.117851 \\
\hline 6 & 0 & -2.817522 & -0.868144 & $4-0.103702$ & 1 & 0 & 1.667846 & 1.806429 & -0.922518 \\
\hline 6 & 0 & -1.484341 & -1.388559 & -0.424587 & 6 & 0 & 1.574161 & 0.809276 & -0.501610 \\
\hline 1 & 0 & -1.423413 & -2.445251 & -0.679901 & 6 & 0 & 2.827075 & 0.122986 & -0.185681 \\
\hline 6 & 0 & -3.198923 & 0.467228 & -0.338540 & 6 & 0 & 4.037544 & 0.802126 & -0.430969 \\
\hline 6 & 0 & -4.476734 & 0.913859 & -0.003576 & 6 & 0 & 5.269377 & 0.215096 & -0.154124 \\
\hline 1 & 0 & -2.499308 & 1.134828 & -0.829922 & 1 & 0 & 4.001698 & 1.808143 & -0.843327 \\
\hline 1 & 0 & -4.750175 & 1.947951 & -0.199089 & 1 & 0 & 6.185321 & 0.765739 & -0.354121 \\
\hline 6 & 0 & -5.410303 & 0.039532 & 0.555936 & 6 & 0 & 5.327603 & -1.074134 & 0.378799 \\
\hline 1 & 0 & -6.407324 & 0.389608 & 0.809649 & 1 & 0 & 6.286899 & -1.536239 & 0.596546 \\
\hline 6 & 0 & -5.056415 & -1.295357 & $7 \quad 0.768046$ & 6 & 0 & 4.138231 & -1.763229 & 0.627541 \\
\hline 1 & 0 & -5.777938 & -1.990129 & 1.190683 & 1 & 0 & 4.170843 & -2.768362 & 1.041440 \\
\hline 6 & 0 & -3.779618 & -1.742706 & $5 \quad 0.438435$ & 6 & 0 & 2.903961 & -1.179418 & 0.350302 \\
\hline 1 & 0 & -3.510964 & -2.782636 & $5 \quad 0.609962$ & 1 & 0 & 1.991317 & -1.728357 & 0.547169 \\
\hline \multicolumn{5}{|c|}{$\begin{array}{c}\alpha E \text {-TS1, } G=-2829.915724 \text { Hartree } \\
1 \text { imaginary frequencies }-97.52\end{array}$} & \multicolumn{5}{|c|}{$\begin{array}{c}\alpha E \text {-TS2, } G=-2829.924017 \text { Hartree } \\
1 \text { imaginary frequencies }-184.93\end{array}$} \\
\hline 6 & 0 & -1.755574 & -0.674539 & 0.988532 & 6 & 0 & -1.899599 & 0.110159 & 0.907077 \\
\hline 6 & 0 & -0.578927 & -0.276266 & $5 \quad 0.093838$ & 6 & 0 & -0.891974 & -0.014293 & -0.255709 \\
\hline 6 & 0 & -2.827027 & -1.209869 & -0.001783 & 6 & 0 & -2.971289 & -0.964463 & 0.582982 \\
\hline 1 & 0 & -2.140851 & 0.280429 & 1.386372 & 1 & 0 & -2.377120 & 1.089034 & 0.743898 \\
\hline 8 & 0 & -1.420188 & -1.513845 & $5 \quad 2.076978$ & 8 & 0 & -1.338273 & 0.095137 & 2.201128 \\
\hline 1 & 0 & -3.853044 & -0.977903 & 30.322856 & 1 & 0 & -3.980881 & -0.609559 & 0.840707 \\
\hline 8 & 0 & -2.667712 & -2.609472 & -0.113567 & 8 & 0 & -2.684634 & -2.144837 & 1.294772 \\
\hline 6 & 0 & -2.475788 & -0.435784 & $4-1.294014$ & 6 & 0 & -2.826382 & -1.111821 & -0.955592 \\
\hline 1 & 0 & -2.336330 & -1.138081 & -2.121567 & 1 & 0 & -2.580231 & -2.150360 & -1.198504 \\
\hline 6 & 0 & -3.479805 & 0.638522 & -1.686499 & 6 & 0 & -4.030144 & -0.654917 & -1.767365 \\
\hline 19 & 0 & 1.291840 & -1.375130 & 2.784180 & 19 & 0 & 1.412334 & -0.056111 & 2.729086 \\
\hline 8 & 0 & -1.204645 & 0.234055 & -1.044947 & 8 & 0 & -1.711806 & -0.274599 & -1.348940 \\
\hline 16 & 0 & 0.477735 & -1.691927 & -0.479839 & 16 & 0 & 0.377841 & -1.475885 & -0.091383 \\
\hline
\end{tabular}




\begin{tabular}{|c|c|c|c|c|c|c|c|c|c|}
\hline 6 & 0 & -2.519629 & -1.788959 & 2.930400 & 6 & 0 & -2.298955 & 0.352172 & 3.218253 \\
\hline 6 & 0 & -3.683010 & -3.245549 & -0.869872 & 6 & 0 & -3.722258 & -3.112662 & 1.238698 \\
\hline 1 & 0 & -3.067637 & 1.240967 & -2.511410 & 1 & 0 & -3.778330 & -0.674997 & -2.839366 \\
\hline 1 & 0 & -3.663423 & 1.321134 & -0.840143 & 1 & 0 & -4.290965 & 0.385264 & -1.504964 \\
\hline 8 & 0 & -4.674495 & -0.013389 & -2.079798 & 8 & 0 & -5.097195 & -1.533623 & -1.474583 \\
\hline 6 & 0 & -5.683235 & 0.889455 & -2.479433 & 6 & 0 & -6.274199 & -1.230990 & -2.197032 \\
\hline 1 & 0 & -2.127213 & -2.341630 & 3.789463 & 1 & 0 & -1.752454 & 0.427140 & 4.163196 \\
\hline 1 & 0 & -3.268049 & -2.409274 & 2.424644 & 1 & 0 & -3.022991 & -0.466410 & 3.292359 \\
\hline 1 & 0 & -2.991577 & -0.860608 & 3.288292 & 1 & 0 & -2.825952 & 1.300835 & 3.037405 \\
\hline 1 & 0 & -3.444125 & -4.311617 & -0.876684 & 1 & 0 & -3.373541 & -3.971675 & 1.815830 \\
\hline 1 & 0 & -3.725521 & -2.879000 & -1.904027 & 1 & 0 & -3.941579 & -3.428226 & 0.210779 \\
\hline 1 & 0 & -4.673835 & -3.095141 & -0.412358 & 1 & 0 & -4.651928 & -2.723830 & 1.683326 \\
\hline 1 & 0 & -6.557440 & 0.295248 & -2.758894 & 1 & 0 & -7.030210 & -1.962339 & -1.900768 \\
\hline 1 & 0 & -5.368900 & 1.492909 & -3.346080 & 1 & 0 & -6.111550 & -1.302432 & -3.283577 \\
\hline 1 & 0 & -5.962050 & 1.576507 & -1.664147 & 1 & 0 & -6.642830 & -0.218931 & -1.966072 \\
\hline 6 & 0 & 2.100676 & -0.864456 & -0.675912 & 6 & 0 & 2.004421 & -0.554745 & -0.280262 \\
\hline 8 & 0 & 0.174212 & -2.156617 & -1.849711 & 8 & 0 & 0.145079 & -2.388225 & -1.222645 \\
\hline 8 & 0 & 0.760286 & -2.678525 & 0.599254 & 8 & 0 & 0.402141 & -2.018427 & 1.288539 \\
\hline 7 & 0 & 2.919949 & -0.693899 & 0.311629 & 7 & 0 & 2.949939 & -0.843948 & 0.594546 \\
\hline 6 & 0 & 4.079208 & -0.060501 & -0.118018 & 6 & 0 & 4.207302 & -0.632785 & 0.056151 \\
\hline 6 & 0 & 5.170884 & 0.276475 & 0.696212 & 6 & 0 & 5.419185 & -0.737021 & 0.754317 \\
\hline 6 & 0 & 4.111444 & 0.245109 & -1.501249 & 6 & 0 & 4.243604 & -0.304051 & -1.321698 \\
\hline 6 & 0 & 5.217928 & 0.876502 & -2.077553 & 6 & 0 & 5.441475 & -0.072601 & -1.991213 \\
\hline 16 & 0 & 2.626433 & -0.300887 & -2.257674 & 16 & 0 & 2.600142 & -0.288624 & -1.967885 \\
\hline 6 & 0 & -0.579748 & 2.457270 & 0.982168 & 6 & 0 & -0.598791 & 2.548487 & -0.350465 \\
\hline 6 & 0 & 0.487255 & 1.444803 & 1.239576 & 6 & 0 & 0.063190 & 1.185546 & -0.519812 \\
\hline 1 & 0 & 1.276821 & 1.431665 & 0.472315 & 1 & 0 & 0.344913 & 1.104059 & -1.587963 \\
\hline 8 & 0 & 0.730963 & 1.047433 & 2.406479 & 8 & 0 & 1.168181 & 0.998227 & 0.300674 \\
\hline 6 & 0 & -1.445534 & 2.838045 & 2.017791 & 6 & 0 & -0.169525 & 3.433307 & 0.643262 \\
\hline 6 & 0 & -2.415052 & 3.817195 & 1.804889 & 6 & 0 & -0.773442 & 4.683948 & 0.792676 \\
\hline 1 & 0 & -1.322716 & 2.367152 & 2.988632 & 1 & 0 & 0.663570 & 3.141891 & 1.274062 \\
\hline 1 & 0 & -3.078931 & 4.109805 & 2.614918 & 1 & 0 & -0.420186 & 5.364789 & 1.563587 \\
\hline 6 & 0 & -2.523435 & 4.434268 & 0.555351 & 6 & 0 & -1.818079 & 5.065518 & -0.049763 \\
\hline 1 & 0 & -3.272758 & 5.204630 & 0.390690 & 1 & 0 & -2.286706 & 6.039515 & 0.064357 \\
\hline 6 & 0 & -1.657392 & 4.067226 & -0.479029 & 6 & 0 & -2.246201 & 4.192192 & -1.052906 \\
\hline 1 & 0 & -1.734026 & 4.550564 & -1.449830 & 1 & 0 & -3.048153 & 4.486557 & -1.725392 \\
\hline 6 & 0 & -0.693659 & 3.082645 & -0.266963 & 6 & 0 & -1.637290 & 2.946563 & -1.206370 \\
\hline 1 & 0 & -0.026415 & 2.787256 & -1.071914 & 1 & 0 & -1.963710 & 2.275376 & -1.995889 \\
\hline 1 & 0 & 5.241691 & 1.107248 & -3.138185 & 1 & 0 & 5.450038 & 0.180067 & -3.047752 \\
\hline 6 & 0 & 6.266556 & 0.906449 & 0.121828 & 6 & 0 & 6.620513 & -0.508560 & 0.085202 \\
\hline 1 & 0 & 5.142665 & 0.043506 & 1.756488 & 1 & 0 & 5.406916 & -1.003821 & 1.808099 \\
\hline 1 & 0 & 7.117853 & 1.174923 & 0.740677 & 1 & 0 & 7.558151 & -0.590747 & 0.629094 \\
\hline 6 & 0 & 6.290196 & 1.202983 & -1.253299 & 6 & 0 & 6.637330 & -0.177215 & -1.275739 \\
\hline 1 & 0 & 7.159145 & 1.695809 & -1.679983 & 1 & 0 & 7.582785 & -0.005163 & -1.782368 \\
\hline \multicolumn{5}{|c|}{$\begin{array}{c}\alpha E-\mathbf{T S 3}, \mathrm{G}=-2829.971345 \text { Hartree } \\
1 \text { imaginary frequencies }-250.49\end{array}$} & \multicolumn{5}{|c|}{$\begin{array}{c}\alpha Z-\mathbf{T S 1}, \mathrm{G}=-2829.910055 \text { Hartree } \\
1 \text { imaginary frequencies }-118.87\end{array}$} \\
\hline 6 & 0 & -2.657668 & 0.219738 & -0.709652 & 6 & 0 & -2.375470 & -0.809157 & 0.898027 \\
\hline 6 & 0 & -1.228908 & 0.123857 & -0.135016 & 6 & 0 & -1.125222 & -0.108466 & 0.336736 \\
\hline 6 & 0 & -3.205074 & -1.237338 & -0.544177 & 6 & 0 & -3.524285 & -0.383030 & -0.057129 \\
\hline 1 & 0 & -2.603397 & 0.433394 & -1.792281 & 1 & 0 & -2.568901 & -0.298810 & 1.859468 \\
\hline 8 & 0 & -3.396509 & 1.225980 & -0.078624 & 8 & 0 & -2.236111 & -2.191587 & 1.147964 \\
\hline 1 & 0 & -3.733807 & -1.557176 & -1.454875 & 1 & 0 & -4.482640 & -0.261769 & 0.471430 \\
\hline 8 & 0 & -4.095130 & -1.270027 & 0.551459 & 8 & 0 & -3.655877 & -1.364786 & -1.062190 \\
\hline 6 & 0 & -1.931318 & -2.094206 & -0.326061 & 6 & 0 & -3.010526 & 0.981957 & -0.573164 \\
\hline 1 & 0 & -1.990824 & -2.579815 & 0.653622 & 1 & 0 & -2.987844 & 0.981371 & -1.666707 \\
\hline 6 & 0 & -1.661251 & -3.133526 & -1.407062 & 6 & 0 & -3.785621 & 2.189481 & -0.066713 \\
\hline 19 & 0 & 1.358532 & 0.169167 & 3.116691 & 19 & 0 & 0.452807 & -2.860893 & 1.455971 \\
\hline 8 & 0 & -0.811279 & -1.196704 & -0.335015 & 8 & 0 & -1.642374 & 1.121061 & -0.087695 \\
\hline 16 & 0 & -1.672478 & 0.225723 & 2.108724 & 16 & 0 & -0.409788 & -0.950470 & -1.161317 \\
\hline
\end{tabular}




\begin{tabular}{|c|c|c|c|c|c|c|c|c|c|}
\hline 6 & 0 & -4.627544 & 1.532362 & -0.710336 & 6 & 0 & -3.379673 & -2.771762 & 1.755944 \\
\hline 6 & 0 & -4.867582 & -2.458546 & 0.622163 & 6 & 0 & -4.773830 & -1.174024 & -1.912132 \\
\hline 1 & 0 & -0.674643 & -3.591533 & -1.234657 & 1 & 0 & -3.255719 & 3.112018 & -0.353273 \\
\hline 1 & 0 & -1.633388 & -2.647662 & -2.398102 & 1 & 0 & -3.843034 & 2.169363 & 1.035830 \\
\hline 8 & 0 & -2.690611 & -4.101558 & -1.348729 & 8 & 0 & -5.078253 & 2.146553 & -0.639807 \\
\hline 6 & 0 & -2.526420 & -5.136847 & -2.295650 & 6 & 0 & -5.888183 & 3.235488 & -0.248820 \\
\hline 1 & 0 & -5.034558 & 2.402641 & -0.190410 & 1 & 0 & -3.120652 & -3.806399 & 1.998796 \\
\hline 1 & 0 & -5.336529 & 0.699752 & -0.630030 & 1 & 0 & -4.234008 & -2.773273 & 1.070140 \\
\hline 1 & 0 & -4.479015 & 1.785910 & -1.771836 & 1 & 0 & -3.651275 & -2.243706 & 2.683563 \\
\hline 1 & 0 & -5.514167 & -2.354907 & 1.496860 & 1 & 0 & -4.755093 & -1.990648 & -2.637416 \\
\hline 1 & 0 & -4.243862 & -3.354713 & 0.731021 & 1 & 0 & -4.733827 & -0.215748 & -2.446476 \\
\hline 1 & 0 & -5.489323 & -2.581838 & -0.278509 & 1 & 0 & -5.716591 & -1.210506 & -1.343452 \\
\hline 1 & 0 & -3.364124 & -5.827271 & -2.167621 & 1 & 0 & -6.855057 & 3.112981 & -0.743856 \\
\hline 1 & 0 & -1.583372 & -5.683551 & -2.138872 & 1 & 0 & -5.448932 & 4.198534 & -0.553777 \\
\hline 1 & 0 & -2.535053 & -4.749748 & -3.327214 & 1 & 0 & -6.043790 & 3.256349 & 0.841940 \\
\hline 6 & 0 & 2.378158 & 0.422625 & 0.237439 & 6 & 0 & 1.378282 & -0.564504 & -1.072230 \\
\hline 8 & 0 & -0.989037 & 1.462001 & 2.659399 & 8 & 0 & -0.820108 & -0.330223 & -2.438214 \\
\hline 8 & 0 & -0.920402 & -1.028816 & 2.500455 & 8 & 0 & -0.404401 & -2.429749 & -1.004419 \\
\hline 7 & 0 & 3.147891 & -0.275792 & 1.047349 & 7 & 0 & 2.200917 & -1.272337 & -0.365907 \\
\hline 6 & 0 & 4.291215 & -0.734493 & 0.416367 & 6 & 0 & 3.506790 & -0.850698 & -0.573588 \\
\hline 6 & 0 & 5.292031 & -1.505109 & 1.024328 & 6 & 0 & 4.645847 & -1.401813 & 0.032562 \\
\hline 6 & 0 & 4.425290 & -0.398429 & -0.953866 & 6 & 0 & 3.645267 & 0.222770 & -1.487506 \\
\hline 6 & 0 & 5.518636 & -0.820501 & -1.707387 & 16 & 0 & 2.068204 & 0.687952 & -2.090139 \\
\hline 16 & 0 & 3.051343 & 0.580468 & -1.452111 & 1 & 0 & -0.947347 & 0.797772 & 2.434749 \\
\hline 6 & 0 & -0.445339 & 2.451035 & -0.889533 & 6 & 0 & 0.031866 & 0.351085 & 2.177855 \\
\hline 6 & 0 & -0.240838 & 1.023973 & -0.680999 & 6 & 0 & 1.039459 & 1.397050 & 1.788558 \\
\hline 1 & 0 & 0.408675 & 0.545136 & -1.411007 & 8 & 0 & 0.395651 & -0.712876 & 2.745983 \\
\hline 8 & 0 & 1.287180 & 0.997623 & 0.584676 & 6 & 0 & 0.637755 & 2.659834 & 1.328558 \\
\hline 6 & 0 & -1.137812 & 3.271831 & 0.022663 & 6 & 0 & 1.583021 & 3.648882 & 1.061152 \\
\hline 6 & 0 & -1.306795 & 4.626725 & -0.252820 & 1 & 0 & -0.417641 & 2.853617 & 1.168371 \\
\hline 1 & 0 & -1.499732 & 2.851387 & 0.951964 & 1 & 0 & 1.259804 & 4.622146 & 0.699928 \\
\hline 1 & 0 & -1.833848 & 5.254659 & 0.460419 & 6 & 0 & 2.942235 & 3.394496 & 1.263564 \\
\hline 6 & 0 & -0.801827 & 5.181676 & -1.431369 & 1 & 0 & 3.678422 & 4.168856 & 1.062120 \\
\hline 1 & 0 & -0.941848 & 6.239457 & -1.638863 & 6 & 0 & 3.349406 & 2.145143 & 1.737628 \\
\hline 6 & 0 & -0.104070 & 4.378641 & -2.338370 & 1 & 0 & 4.404473 & 1.943527 & 1.905530 \\
\hline 1 & 0 & 0.300143 & 4.807083 & -3.251310 & 6 & 0 & 2.402790 & 1.155999 & 2.001126 \\
\hline 6 & 0 & 0.084793 & 3.028878 & -2.062017 & 1 & 0 & 2.698633 & 0.188487 & 2.393220 \\
\hline 1 & 0 & 0.641792 & 2.406938 & -2.758379 & 6 & 0 & 5.892161 & -0.875238 & -0.278216 \\
\hline 1 & 0 & 5.606092 & -0.556884 & -2.757531 & 1 & 0 & 4.538049 & -2.225796 & 0.731944 \\
\hline 6 & 0 & 6.387233 & -1.924599 & 0.273385 & 1 & 0 & 6.782645 & -1.291859 & 0.183720 \\
\hline 1 & 0 & 5.196835 & -1.769652 & 2.073889 & 6 & 0 & 6.018881 & 0.191696 & -1.187261 \\
\hline 1 & 0 & 7.161601 & -2.523305 & 0.745631 & 1 & 0 & 7.005006 & 0.585978 & -1.415807 \\
\hline 6 & 0 & 6.503235 & -1.587190 & -1.082367 & 6 & 0 & 4.901955 & 0.749965 & -1.800162 \\
\hline 1 & 0 & 7.364621 & -1.922338 & -1.653249 & 1 & 0 & 5.005250 & 1.573046 & -2.500510 \\
\hline \multicolumn{5}{|c|}{$\begin{array}{c}\alpha Z \text {-TS2, } G=-2829.920174 \text { Hartree } \\
1 \text { imaginary frequencies }-194.18\end{array}$} & \multicolumn{5}{|c|}{$\begin{array}{c}\alpha Z-\mathbf{T S 3}, \mathrm{G}=-2829.950645 \text { Hartree } \\
1 \text { imaginary frequencies }-338.21\end{array}$} \\
\hline 6 & 0 & 2.317888 & -0.449019 & -1.117614 & 6 & 0 & -2.196177 & -0.706364 & 1.247367 \\
\hline 6 & 0 & 0.920151 & -0.125051 & -0.574277 & 6 & 0 & -1.301491 & 0.213496 & 0.409289 \\
\hline 6 & 0 & 3.013974 & -1.208692 & 0.048139 & 6 & 0 & -3.488679 & -0.858413 & 0.401912 \\
\hline 1 & 0 & 2.825673 & 0.526998 & -1.218708 & 1 & 0 & -2.474363 & -0.153660 & 2.165972 \\
\hline 8 & 0 & 2.254307 & -1.095050 & -2.354934 & 8 & 0 & -1.570652 & -1.913133 & 1.656198 \\
\hline 1 & 0 & 4.065205 & -0.898932 & 0.150838 & 1 & 0 & -4.386996 & -0.806614 & 1.037958 \\
\hline 8 & 0 & 2.946204 & -2.590473 & -0.208628 & 8 & 0 & -3.458235 & -2.100869 & -0.264610 \\
\hline 6 & 0 & 2.199511 & -0.756241 & 1.287148 & 6 & 0 & -3.416753 & 0.372826 & -0.540495 \\
\hline 1 & 0 & 1.778010 & -1.634039 & 1.785028 & 1 & 0 & -3.324735 & 0.027224 & -1.572974 \\
\hline 6 & 0 & 2.965505 & 0.102158 & 2.284923 & 6 & 0 & -4.569383 & 1.358020 & -0.405352 \\
\hline 19 & 0 & -2.530535 & -0.071913 & -3.075768 & 19 & 0 & 0.681059 & -3.005874 & 0.666455 \\
\hline 8 & 0 & 1.109253 & 0.066759 & 0.797326 & 8 & 0 & -2.227380 & 1.105554 & -0.180015 \\
\hline 16 & 0 & -0.393436 & -1.472874 & -0.730052 & 16 & 0 & -0.287180 & -0.593233 & -1.485455 \\
\hline
\end{tabular}




\begin{tabular}{|c|c|c|c|c|c|c|c|}
\hline 6 & 0 & 3.515601 & $-1.285187-2.975165$ & 6 & 0 & -2.358106 & $-2.654366 \quad 2.578073$ \\
\hline 6 & 0 & 3.726858 & $\begin{array}{ll}-3.378186 & 0.675278\end{array}$ & 6 & 0 & -4.669448 & $-2.425502-0.927843$ \\
\hline 1 & 0 & 2.272089 & $0.483239 \quad 3.050798$ & 1 & 0 & -4.362692 & $2.247561 \quad-1.020652$ \\
\hline 1 & 0 & 3.405545 & $0.974896 \quad 1.772843$ & 1 & 0 & -4.658945 & $1.692308 \quad 0.643275$ \\
\hline 8 & 0 & 3.968390 & $-0.710891 \quad 2.861164$ & 8 & 0 & -5.753124 & $0.709596-0.829402$ \\
\hline 6 & 0 & 4.733864 & $-0.031090 \quad 3.835199$ & 6 & 0 & -6.885364 & $1.551634-0.786010$ \\
\hline 1 & 0 & 3.314370 & $-1.710951-3.960865$ & 1 & 0 & -1.753167 & $-3.504267 \quad 2.911602$ \\
\hline 1 & 0 & 4.140490 & $-1.981109 \quad-2.404017$ & 1 & 0 & -3.272204 & $-3.029378 \quad 2.105611$ \\
\hline 1 & 0 & 4.049893 & $-0.329212-3.098056$ & 1 & 0 & -2.619221 & $-2.045104 \quad 3.456806$ \\
\hline 1 & 0 & 3.587303 & $-4.416700 \quad 0.367222$ & 1 & 0 & -4.514120 & $-3.401907-1.393053$ \\
\hline 1 & 0 & 3.413989 & $-3.265558 \quad 1.721337$ & 1 & 0 & -4.929749 & $-1.691327-1.699556$ \\
\hline 1 & 0 & 4.794271 & $-3.114732 \quad 0.606588$ & 1 & 0 & -5.509578 & $-2.486464-0.217206$ \\
\hline 1 & 0 & 5.470302 & $-0.741469 \quad 4.219398$ & 1 & 0 & -7.739283 & $0.963490-1.132408$ \\
\hline 1 & 0 & 4.106779 & $0.322135 \quad 4.668634$ & 1 & 0 & -6.764218 & $2.428323-1.441637$ \\
\hline 1 & 0 & 5.260676 & $0.835874 \quad 3.405629$ & 1 & 0 & -7.087863 & $1.909753 \quad 0.236500$ \\
\hline 6 & 0 & -1.790301 & $-0.402823 \quad-0.094083$ & 6 & 0 & 2.306547 & $-0.291665 \quad 0.640206$ \\
\hline 8 & 0 & -0.130339 & $-2.603172 \quad 0.170545$ & 8 & 0 & -1.183834 & $0.069600 \quad-2.481878$ \\
\hline 8 & 0 & -0.699140 & $-1.727391 \quad-2.165050$ & 8 & 0 & -0.443681 & $-2.098061-1.409525$ \\
\hline 7 & 0 & -2.998223 & $-0.727207-0.549670$ & 7 & 0 & 2.932782 & $-1.403953 \quad 0.327550$ \\
\hline 6 & 0 & -3.985797 & $-0.488952 \quad 0.389171$ & 6 & 0 & 4.210896 & $-1.156755-0.147659$ \\
\hline 6 & 0 & -5.363493 & $-0.637343 \quad 0.174076$ & 6 & 0 & 5.121952 & $-2.136482-0.564483$ \\
\hline 6 & 0 & -3.539492 & $-0.075615 \quad 1.667686$ & 6 & 0 & 4.588123 & $0.204975-0.212140$ \\
\hline 16 & 0 & -1.775047 & $-0.001202 \quad 1.681904$ & 16 & 0 & 3.266312 & $1.209620 \quad 0.371583$ \\
\hline 1 & 0 & 0.421074 & $0.936645-2.322931$ & 1 & 0 & -0.283822 & $0.769463 \quad 2.234924$ \\
\hline 6 & 0 & 0.194959 & $1.075883-1.249365$ & 6 & 0 & -0.243904 & $0.904748 \quad 1.156402$ \\
\hline 6 & 0 & 0.765287 & $2.422551 \quad-0.829472$ & 6 & 0 & 0.225044 & $2.243250 \quad 0.774425$ \\
\hline 8 & 0 & -1.197950 & $0.988736-1.037178$ & 8 & 0 & $1.126330-$ & $-0.308764 \quad 1.187633$ \\
\hline 6 & 0 & 1.731364 & $3.060049-1.617397$ & 6 & 0 & 0.761276 & $3.068627 \quad 1.782862$ \\
\hline 6 & 0 & 2.264965 & $4.294166-1.236964$ & 6 & 0 & 1.204925 & $\begin{array}{ll}4.354428 & 1.492928\end{array}$ \\
\hline 1 & 0 & 2.061888 & $2.594156-2.544358$ & 1 & 0 & 0.831377 & $2.687818 \quad 2.799051$ \\
\hline 1 & 0 & 3.011691 & $4.777198 \quad-1.862252$ & 1 & 0 & 1.611520 & $4.980017 \quad 2.282632$ \\
\hline 6 & 0 & 1.826750 & $4.908344-0.064031$ & 6 & 0 & 1.131152 & $4.833151 \quad 0.181879$ \\
\hline 1 & 0 & 2.233645 & $5.871528 \quad 0.233152$ & 1 & 0 & 1.481042 & $5.835652-0.050388$ \\
\hline 6 & 0 & 0.851550 & $4.284622 \quad 0.720118$ & 6 & 0 & 0.603507 & $4.025324-0.828195$ \\
\hline 1 & 0 & 0.498877 & $4.764005 \quad 1.629923$ & 1 & 0 & 0.541431 & $4.398635-1.846435$ \\
\hline 6 & 0 & 0.322162 & $3.052226 \quad 0.340731$ & 6 & 0 & 0.147546 & $2.740789-0.542310$ \\
\hline 1 & 0 & -0.446098 & $2.571651 \quad 0.937149$ & 1 & 0 & -0.284513 & $2.133846-1.329152$ \\
\hline 6 & 0 & -6.257060 & $-0.365951 \quad 1.210416$ & 6 & 0 & 6.373960 & $-1.748685-1.034243$ \\
\hline 1 & 0 & -5.720660 & $-0.974735-0.796156$ & 1 & 0 & $4.838738-$ & $-3.184561 \quad-0.519598$ \\
\hline 1 & 0 & -7.324092 & $-0.484825 \quad 1.039352$ & 1 & 0 & 7.081572 & $-2.506512-1.359652$ \\
\hline 6 & 0 & -5.799662 & $0.054315 \quad 2.464708$ & 6 & 0 & $6.732559-$ & $-0.394322-1.096824$ \\
\hline 1 & 0 & -6.507709 & $0.260041 \quad 3.262561$ & 1 & 0 & 7.713620 & $-0.110471 \quad-1.467090$ \\
\hline 6 & 0 & -4.429025 & $0.203636 \quad 2.699948$ & 6 & 0 & 5.838712 & $0.595707-0.687748$ \\
\hline 1 & 0 & -4.068911 & $0.524974 \quad 3.673514$ & 1 & 0 & 6.113693 & $1.645361 \quad-0.737539$ \\
\hline \multicolumn{4}{|c|}{$\begin{array}{c}\beta E-\text { TS1, } G=-2829.910992 \text { Hartree } \\
1 \text { imaginary frequencies }-174.67\end{array}$} & \multicolumn{4}{|c|}{$\begin{array}{c}\beta E-\mathbf{T S 2}, \mathrm{G}=-2829.922188 \text { Hartree } \\
1 \text { imaginary frequencies }-179.37\end{array}$} \\
\hline 6 & 0 & -1.757895 & \begin{tabular}{|lll}
5 & 0.347482 & 1.273275
\end{tabular} & 6 & 0 & 1.848518 & $-0.343543 \quad 0.977211$ \\
\hline 6 & 0 & -0.607946 & $6 \quad 0.319080 \quad 0.248094$ & 6 & 0 & 0.889407 & $-0.324480 \quad-0.241342$ \\
\hline 8 & 0 & -1.110458 & $\begin{array}{lll}3 & 0.779007 & -0.977725\end{array}$ & 8 & 0 & 1.623629 & $-0.772730 \quad-1.336940$ \\
\hline 6 & 0 & -3.035504 & $4 \quad 0.497055 \quad 0.397364$ & 6 & 0 & 3.256564 & $-0.387477 \quad 0.339742$ \\
\hline 8 & 0 & -1.699873 & $3-0.799572 \quad 2.115272$ & 8 & 0 & 1.567775 & $0.700984 \quad 1.888314$ \\
\hline 19 & 0 & 1.102054 & $-1.150764 \quad 2.887859$ & 19 & 0 & -1.339826 & $5 \quad 0.410715 \quad 2.734648$ \\
\hline 16 & 0 & 0.633034 & $1.569671 \quad 0.840381$ & 16 & 0 & -0.515391 & $-1.612361 \quad 0.158985$ \\
\hline 6 & 0 & -2.488552 & $2 \quad 1.178950 \quad-0.872291$ & 6 & 0 & 2.963814 & $-1.148938-0.966476$ \\
\hline 8 & 0 & -3.589388 & $\begin{array}{lll}3 & -0.778133 & 0.158689\end{array}$ & 8 & 0 & 3.719200 & $0.927854 \quad 0.129762$ \\
\hline 6 & 0 & -2.710520 & $-0.810762 \quad 3.113407$ & 6 & 0 & 2.471223 & $0.745122 \quad 2.985719$ \\
\hline 8 & 0 & 0.778865 & $1.389958 \quad 2.315980$ & 8 & 0 & -0.472400 & $-1.869309 \quad 1.630989$ \\
\hline 8 & 0 & 0.904106 & $-2.115732 \quad 0.664945$ & 8 & 0 & -1.069681 & $0.936932 \quad 0.231994$ \\
\hline 6 & 0 & 2.240183 & $1.034780 \quad 0.177064$ & 6 & 0 & -2.068447 & $7-0.617363-0.111253$ \\
\hline
\end{tabular}




\begin{tabular}{|c|c|c|c|c|c|c|c|c|}
\hline 6 & 0 & -2.606768 & 2.703333 & -0.860982 & 6 & 0 & 3.071730 & $-2.670824 \quad-0.858297$ \\
\hline 6 & 0 & -4.895763 & -0.741793 & -0.385489 & 6 & 0 & 5.097323 & $1.013801-0.188299$ \\
\hline 6 & 0 & 0.310331 & -1.529716 & -0.289665 & 6 & 0 & 0.026047 & $0.918854-0.618777$ \\
\hline 7 & 0 & 3.001626 & 0.204585 & 0.808512 & 7 & 0 & -2.998461 & $-0.696626 \quad 0.821177$ \\
\hline 16 & 0 & 2.832776 & 1.678330 & -1.350530 & 16 & 0 & -2.694186 & $-0.525145 \quad-1.802034$ \\
\hline 8 & 0 & -3.989496 & 3.013059 & -0.925210 & 8 & 0 & 4.433517 & $-2.974010 \quad-0.619381$ \\
\hline 6 & 0 & -0.855716 & -2.214868 & -0.957757 & 6 & 0 & 0.769706 & $2.246759-0.712635$ \\
\hline 6 & 0 & 4.168398 & -0.020348 & 0.091569 & 6 & 0 & -4.251569 & $-0.443268 \quad 0.288146$ \\
\hline 6 & 0 & 4.265646 & 0.693412 & -1.128826 & 6 & 0 & -4.307995 & $-0.301470 \quad-1.120434$ \\
\hline 6 & 0 & -4.237758 & 4.403329 & -0.924213 & 6 & 0 & 4.678436 & $-4.365039-0.541347$ \\
\hline 6 & 0 & -1.216779 & -1.910247 & -2.276212 & 6 & 0 & 1.651984 & $2.478857-1.775855$ \\
\hline 6 & 0 & -1.522005 & -3.253519 & -0.296733 & 6 & 0 & 0.538575 & $3.276226 \quad 0.203123$ \\
\hline 6 & 0 & 5.210765 & -0.872479 & 0.486204 & 6 & 0 & -5.441087 & $-0.337047 \quad 1.023296$ \\
\hline 6 & 0 & 5.386928 & 0.567983 & -1.954921 & 6 & 0 & -5.503424 & $-0.048779-1.786852$ \\
\hline 6 & 0 & -2.238949 & -2.614854 & -2.914706 & 6 & 0 & 2.308724 & $3.702355-1.903352$ \\
\hline 6 & 0 & -2.540597 & -3.960105 & -0.930588 & 6 & 0 & 1.189591 & $4.503820 \quad 0.076034$ \\
\hline 6 & 0 & 6.320894 & -0.997837 & -0.336860 & 6 & 0 & -6.640164 & $-0.087830 \quad 0.357884$ \\
\hline 6 & 0 & 6.408055 & -0.283367 & -1.546298 & 6 & 0 & -6.676304 & $0.057664-1.035040$ \\
\hline 6 & 0 & -2.905371 & -3.641455 & -2.242560 & 6 & 0 & 2.082347 & $4.720251 \quad-0.974651$ \\
\hline 1 & 0 & -1.715859 & 1.231466 & 1.929258 & 1 & 0 & 1.717310 & $-1.299529 \quad 1.503136$ \\
\hline 1 & 0 & -3.781278 & 1.142776 & 0.883938 & 1 & 0 & 3.968179 & $-0.949543 \quad 0.961131$ \\
\hline 8 & 0 & 0.452945 & 2.932723 & 0.292384 & 8 & 0 & -0.417595 & $-2.759828 \quad-0.759320$ \\
\hline 1 & 0 & -2.996750 & 0.794178 & -1.761626 & 1 & 0 & 3.620441 & $-0.808080-1.771733$ \\
\hline 1 & 0 & -2.513665 & -1.675148 & 3.755000 & 1 & 0 & 2.111100 & $1.529717 \quad 3.657310$ \\
\hline 1 & 0 & -3.705456 & -0.921384 & 2.669723 & 1 & 0 & 3.482167 & $1.003196 \quad 2.654893$ \\
\hline 1 & 0 & -2.682653 & 0.106087 & 3.724924 & 1 & 0 & 2.495018 & $-0.214380 \quad 3.526898$ \\
\hline 1 & 0 & -2.065435 & 3.113744 & -1.726975 & 1 & 0 & 2.715384 & $-3.120812-1.796930$ \\
\hline 1 & 0 & -2.144924 & 3.132741 & 0.040764 & 1 & 0 & 2.430678 & $-3.061549-0.051964$ \\
\hline 1 & 0 & -5.201858 & -1.781026 & -0.522121 & 1 & 0 & 5.321899 & $2.076267 \quad-0.304227$ \\
\hline 1 & 0 & -4.930227 & -0.231243 & -1.357895 & 1 & 0 & 5.345476 & $0.493154-1.124141$ \\
\hline 1 & 0 & -5.599945 & -0.235088 & 0.294334 & 1 & 0 & 5.721222 & $0.593428 \quad 0.616090$ \\
\hline 1 & 0 & 0.910727 & -0.953963 & -1.021630 & 1 & 0 & -0.293146 & $0.690066-1.656060$ \\
\hline 1 & 0 & -5.321752 & 4.538469 & -0.973868 & 1 & 0 & 5.748830 & $-4.493590-0.360898$ \\
\hline 1 & 0 & -3.774940 & 4.899475 & -1.792033 & 1 & 0 & 4.407104 & $-4.875985-1.477901$ \\
\hline 1 & 0 & -3.857170 & 4.884740 & -0.009269 & 1 & 0 & 4.115484 & $-4.830468 \quad 0.282795$ \\
\hline 1 & 0 & -0.689617 & -1.119254 & -2.801841 & 1 & 0 & 1.819762 & $1.695191 \quad-2.509658$ \\
\hline 1 & 0 & -1.215846 & -3.496137 & 0.715323 & 1 & 0 & -0.174337 & $3.116009 \quad 1.004711$ \\
\hline 1 & 0 & 5.130926 & -1.420759 & 1.419803 & 1 & 0 & -5.414165 & $-0.459074 \quad 2.103124$ \\
\hline 1 & 0 & 5.459665 & 1.115824 & -2.889316 & 1 & 0 & -5.527603 & $0.059872 \quad-2.867417$ \\
\hline 1 & 0 & -2.505527 & -2.372318 & -3.940918 & 1 & 0 & 2.987244 & $3.865727-2.737206$ \\
\hline 1 & 0 & -3.048495 & -4.767512 & -0.407347 & 1 & 0 & 0.991499 & $5.296833 \quad 0.793661$ \\
\hline 1 & 0 & 7.133797 & -1.657197 & -0.047013 & 1 & 0 & -7.560898 & $-0.006388 \quad 0.929876$ \\
\hline 1 & 0 & 7.287070 & -0.399319 & -2.173883 & 1 & 0 & -7.620093 & $0.249192-1.537654$ \\
\hline 1 & 0 & -3.694229 & -4.199572 & -2.741771 & 1 & 0 & 2.585053 & $5.678591-1.078239$ \\
\hline \multicolumn{5}{|c|}{$\begin{array}{c}\beta E-\mathbf{T S 3}, \mathrm{G}=-2829.972580 \text { Hartree } \\
1 \text { imaginary frequencies }-185.18\end{array}$} & \multicolumn{4}{|c|}{$\begin{array}{c}\beta Z \text {-TS1, } \mathrm{G}=-2829.912038 \text { Hartree } \\
1 \text { imaginary frequencies }-187.44\end{array}$} \\
\hline 6 & 0 & 2.716255 & -0.063312 & -0.393832 & 6 & 0 & -2.335495 & $-1.128461 \quad-0.275540$ \\
\hline 6 & 0 & 1.330831 & $0.056043 \quad 0$ & 0.273202 & 6 & 0 & -1.083137 & $-0.237378 \quad-0.077993$ \\
\hline 8 & 0 & 0.716931 & -1.207729 & 0.112795 & 8 & 0 & -1.539611 & $1.006341 \quad 0.385744$ \\
\hline 6 & 0 & 2.442621 & -1.096349 & -1.515645 & 6 & 0 & -3.489185 & $-0.096104-0.365242$ \\
\hline 8 & 0 & 3.214398 & $1.178104-$ & -0.795021 & 8 & 0 & -2.177150 & $-1.996602-1.388032$ \\
\hline 19 & 0 & -0.790411 & -1.274922 & 2.603706 & 19 & 0 & 0.585088 & $-3.110676-1.362636$ \\
\hline 16 & 0 & 2.044996 & $0.072478 \quad 2$ & 2.400143 & 16 & 0 & -0.130346 & $-1.037073 \quad 1.312082$ \\
\hline 6 & 0 & 1.424020 & $-2.029647-$ & -0.844943 & 6 & 0 & -2.967997 & $1.025981 \quad 0.546458$ \\
\hline 8 & 0 & 1.895327 & $-0.403734-$ & -2.621667 & 8 & 0 & -3.642069 & $0.317503-1.711710$ \\
\hline 6 & 0 & 4.546750 & $1.138050-$ & -1.274146 & 6 & 0 & -3.302924 & $-2.830533-1.610685$ \\
\hline 8 & 0 & 1.982645 & -1.429921 & 2.563911 & 8 & 0 & -0.160076 & $-2.512114 \quad 1.081864$ \\
\hline 8 & 0 & -1.189566 & 0.927473 & 1.097101 & 8 & 0 & 0.626759 & $-0.853481 \quad-2.263246$ \\
\hline 6 & 0 & -2.273252 & 0.494121 & 0.587849 & 6 & 0 & 1.621887 & $-0.606754 \quad 1.096388$ \\
\hline
\end{tabular}




\begin{tabular}{|c|c|c|c|c|c|c|c|c|}
\hline 6 & 0 & 2.034982 & $-3.240298-0.141180$ & 6 & 0 & -3.361398 & 0.880271 & 2.017420 \\
\hline 6 & 0 & 1.890500 & $\begin{array}{ll}-1.153901 & -3.824209\end{array}$ & 6 & 0 & -4.859112 & 0.998999 & -1.962677 \\
\hline 6 & 0 & 0.443523 & $1.096042-0.202990$ & 6 & 0 & -0.147109 & 0.090635 & -1.892404 \\
\hline 7 & 0 & -2.894067 & $-0.626217 \quad 0.915886$ & 7 & 0 & 2.420750 & -1.323120 & 0.375344 \\
\hline 16 & 0 & -3.147487 & $1.452662 \quad-0.701274$ & 16 & 0 & 2.312113 & 0.752479 & 1.967475 \\
\hline 8 & 0 & 2.578456 & $-4.074387-1.148424$ & 8 & 0 & -4.767632 & 1.053287 & 2.089746 \\
\hline 6 & 0 & 0.682369 & $2.524898-0.182885$ & 6 & 0 & 0.442024 & 1.473530 & -1.722657 \\
\hline 6 & 0 & -4.072792 & $-0.797333 \quad 0.214919$ & 6 & 0 & 3.709235 & -0.812406 & 0.437935 \\
\hline 6 & 0 & -4.389793 & $0.211105 \quad-0.732441$ & 6 & 0 & 3.856507 & 0.333470 & 1.259409 \\
\hline 6 & 0 & 3.176691 & $-5.243424-0.626278$ & 6 & 0 & -5.259389 & 0.971215 & 3.411638 \\
\hline 6 & 0 & 0.206795 & $3.283358-1.273031$ & 6 & 0 & -0.367037 & 2.617480 & -1.739988 \\
\hline 6 & 0 & 1.344507 & $3.187308 \quad 0.869478$ & 6 & 0 & 1.831797 & 1.634193 & -1.659367 \\
\hline 6 & 0 & -4.947570 & $-1.883721 \quad 0.365506$ & 6 & 0 & 4.824842 & -1.339948 & -0.230787 \\
\hline 6 & 0 & -5.541714 & $0.140045-1.514040$ & 6 & 0 & 5.099064 & 0.956030 & 1.417872 \\
\hline 6 & 0 & 0.430829 & $4.654191-1.335253$ & 6 & 0 & 0.197071 & 3.890701 & -1.656079 \\
\hline 6 & 0 & 1.546603 & $4.561801 \quad 0.811742$ & 6 & 0 & 2.399755 & 2.904647 & -1.572693 \\
\hline 6 & 0 & -6.098522 & $-1.952843 \quad-0.414342$ & 6 & 0 & 6.055682 & -0.719339 & -0.072463 \\
\hline 6 & 0 & -6.396640 & $-0.950229-1.348681$ & 6 & 0 & 6.191090 & 0.418580 & 0.745189 \\
\hline 6 & 0 & 1.103041 & $5.296659-0.292013$ & 6 & 0 & 1.582849 & 4.038524 & -1.563728 \\
\hline 1 & 0 & 3.416787 & $-0.537597 \quad 0.316743$ & 1 & 0 & -2.544863 & -1.756173 & 0.605400 \\
\hline 1 & 0 & 3.347920 & $-1.643624-1.812019$ & 1 & 0 & -4.436946 & -0.502415 & 0.013827 \\
\hline 8 & 0 & 0.996748 & $0.766350 \quad 3.226580$ & 8 & 0 & -0.466151 & -0.509496 & $5 \quad 2.653209$ \\
\hline 1 & 0 & 0.691477 & $-2.384698-1.577656$ & 1 & 0 & -3.312622 & 2.003579 & 0.194014 \\
\hline 1 & 0 & 4.849064 & $2.174743-1.438191$ & 1 & 0 & -3.033550 & -3.518642 & -2.417454 \\
\hline 1 & 0 & 4.620588 & $0.590235 \quad-2.223553$ & 1 & 0 & -4.177024 & -2.248416 & -1.925144 \\
\hline 1 & 0 & 5.224972 & $0.676945-0.539427$ & 1 & 0 & -3.558952 & -3.412398 & -0.710497 \\
\hline 1 & 0 & 1.245896 & $\begin{array}{ll}-3.765539 & 0.422193\end{array}$ & 1 & 0 & -2.833136 & 1.645431 & 2.605928 \\
\hline 1 & 0 & 2.798969 & $-2.931983 \quad 0.586757$ & 1 & 0 & -3.060623 & -0.098309 & 2.419612 \\
\hline 1 & 0 & 1.461191 & $-0.508798 \quad-4.594521$ & 1 & 0 & -4.867162 & 1.244308 & -3.027374 \\
\hline 1 & 0 & 1.284972 & $-2.068212-3.746093$ & 1 & 0 & -4.947266 & 1.927753 & -1.381545 \\
\hline 1 & 0 & 2.911415 & $-1.440192-4.120112$ & 1 & 0 & -5.728142 & 0.363655 & -1.730014 \\
\hline 1 & 0 & -0.254913 & $0.759004-0.962820$ & 1 & 0 & -1.163771 & 0.139859 & -2.317591 \\
\hline 1 & 0 & 3.571643 & $-5.810067-1.473525$ & 1 & 0 & -6.342054 & 1.116865 & 3.363672 \\
\hline 1 & 0 & 2.445171 & $\begin{array}{ll}-5.867048 & -0.088237\end{array}$ & 1 & 0 & -4.821061 & 1.749125 & 4.056427 \\
\hline 1 & 0 & 4.001497 & $-5.004335 \quad 0.063283$ & 1 & 0 & -5.048398 & -0.010638 & 3.864479 \\
\hline 1 & 0 & -0.323949 & $2.781249-2.076937$ & 1 & 0 & -1.443129 & 2.502258 & -1.832019 \\
\hline 1 & 0 & 1.638110 & $2.635101 \quad 1.752730$ & 1 & 0 & 2.450678 & 0.744016 & -1.707346 \\
\hline 1 & 0 & -4.713404 & $-2.659030 \quad 1.090066$ & 1 & 0 & 4.708127 & -2.216150 & -0.861327 \\
\hline 1 & 0 & -5.772310 & $0.919014 \quad-2.235197$ & 1 & 0 & 5.209480 & 1.834683 & 2.045750 \\
\hline 1 & 0 & 0.072598 & $5.223627-2.188210$ & 1 & 0 & -0.443750 & 4.769474 & -1.670196 \\
\hline 1 & 0 & 2.045847 & $5.065412 \quad 1.634799$ & 1 & 0 & 3.481132 & 3.012543 & -1.523297 \\
\hline 1 & 0 & -6.775030 & $-2.795267-0.296602$ & 1 & 0 & 6.927286 & -1.113741 & -0.586617 \\
\hline 1 & 0 & -7.300263 & $-1.018949-1.947923$ & 1 & 0 & 7.165208 & 0.887265 & 0.852211 \\
\hline 1 & 0 & 1.268181 & $6.370169-0.331994$ & 1 & 0 & 2.023667 & 5.030747 & -1.501782 \\
\hline \multirow{2}{*}{\multicolumn{4}{|c|}{$\begin{array}{c}\beta Z \text {-TS2, } \mathrm{G}=-2829.922954 \text { Hartree } \\
1 \text { imaginary frequencies }-191.04\end{array}$}} & \multirow{2}{*}{\multicolumn{5}{|c|}{$\begin{array}{c}\beta Z \text {-TS3, } G=-2829.963736 \text { Hartree } \\
1 \text { imaginary frequencies }-285.54\end{array}$}} \\
\hline & & & & & & & & \\
\hline 6 & 0 & -2.210252 & $2 \quad 0.270026 \quad 1.354154$ & 6 & 0 & -2.164391 & -1.155741 & -0.525688 \\
\hline 6 & 0 & -0.909225 & $5 \quad 0.023604 \quad 0.546594$ & 6 & 0 & -1.217661 & -0.125305 & 0.097734 \\
\hline 8 & 0 & -1.077371 & $1 \quad 0.607666-0.713782$ & 8 & 0 & -2.016148 & 0.637094 & 0.984422 \\
\hline 6 & 0 & -3.244254 & $\begin{array}{lll}4 & 0.721915 & 0.281400\end{array}$ & 6 & 0 & -3.508738 & -0.397492 & -0.568722 \\
\hline 8 & 0 & -2.560977 & $7-0.861752 \quad 2.105311$ & 8 & 0 & -1.677950 & -1.633743 & -1.772427 \\
\hline 19 & 0 & 2.300126 & $-1.680036 \quad 2.714139$ & 19 & 0 & 1.085592 & -2.710601 & -1.624173 \\
\hline 16 & 0 & 0.488002 & $0.951904 \quad 1.381638$ & 16 & 0 & -0.091464 & -1.310440 & 1.510595 \\
\hline 6 & 0 & -2.355767 & $7 \quad 1.257944-0.855150$ & 6 & 0 & -3.417357 & 0.460369 & 0.706291 \\
\hline 8 & 0 & -4.023785 & $5 \quad-0.393151 \quad-0.094592$ & 8 & 0 & -3.537254 & 0.380915 & -1.756968 \\
\hline 6 & 0 & -3.615249 & $-0.630718 \quad 3.026645$ & 6 & 0 & -2.562036 & -2.548835 & -2.403976 \\
\hline 8 & 0 & 0.641256 & $0.389961 \quad 2.756280$ & 8 & 0 & 0.162957 & -2.561978 & 0.682665 \\
\hline 8 & 0 & 1.096777 & $-1.345879 \quad 0.416511$ & 8 & 0 & 1.196665 & -0.100045 & -1.209701 \\
\hline 6 & 0 & 1.876263 & $0.282732 \quad 0.355250$ & 6 & 0 & 2.346250 & -0.091524 & -0.608515 \\
\hline
\end{tabular}




\begin{tabular}{|c|c|c|c|c|c|c|c|}
\hline 6 & 0 & -2.184824 & $2.776522-0.852710$ & 6 & 0 & -4.087752 & $-0.163177 \quad 1.928837$ \\
\hline 6 & 0 & -5.185817 & $-0.050885-0.830740$ & 6 & 0 & -4.813845 & $0.920485-2.052653$ \\
\hline 6 & 0 & -0.318656 & $-1.423603 \quad 0.458562$ & 6 & 0 & -0.467759 & $0.705776-0.850703$ \\
\hline 7 & 0 & 3.055628 & $0.192097 \quad 0.960096$ & 7 & 0 & 3.176092 & $-1.108021 \quad-0.684887$ \\
\hline 16 & 0 & 1.973593 & $0.872165-1.360022$ & 16 & 0 & 2.989415 & $1.335586 \quad 0.289236$ \\
\hline 8 & 0 & -3.447811 & $3.323319-1.192929$ & 8 & 0 & -5.485164 & $-0.171353 \quad 1.679944$ \\
\hline 6 & 0 & -0.888757 & $-2.235925 \quad-0.698447$ & 6 & 0 & -0.184493 & $2.125873-0.609214$ \\
\hline 6 & 0 & 4.099561 & $0.374483 \quad 0.068848$ & 6 & 0 & 4.374869 & $-0.840678-0.043806$ \\
\hline 6 & 0 & 3.731749 & $0.742518-1.247844$ & 6 & 0 & 4.475513 & $0.433205 \quad 0.564582$ \\
\hline 6 & 0 & -3.437969 & $4.736200-1.233136$ & 6 & 0 & -6.222759 & $-0.724748 \quad 2.749635$ \\
\hline 6 & 0 & -2.108011 & $-2.909908 \quad-0.556430$ & 6 & 0 & -0.088065 & $2.692950 \quad 0.676693$ \\
\hline 6 & 0 & -0.183513 & $-2.350162-1.901111$ & 6 & 0 & 0.008419 & $2.955583-1.730552$ \\
\hline 6 & 0 & 5.461099 & $0.238940 \quad 0.374011$ & 6 & 0 & 5.457096 & $-1.726129 \quad 0.047505$ \\
\hline 6 & 0 & 4.680927 & $0.953000 \quad-2.242846$ & 6 & 0 & 5.620121 & $0.823723 \quad 1.257313$ \\
\hline 6 & 0 & -2.616970 & $-3.679769-1.603323$ & 6 & 0 & 0.184414 & $4.050763 \quad 0.824376$ \\
\hline 6 & 0 & -0.693627 & $-3.119084-2.947911$ & 6 & 0 & 0.273351 & $4.312718-1.579347$ \\
\hline 6 & 0 & 6.415208 & $0.457574 \quad-0.619829$ & 6 & 0 & 6.603191 & $-1.335779 \quad 0.735138$ \\
\hline 6 & 0 & 6.033898 & $0.809444 \quad-1.919894$ & 6 & 0 & 6.687270 & $-0.071736 \quad 1.336530$ \\
\hline 6 & 0 & -1.911285 & $-3.787156-2.803062$ & 6 & 0 & 0.363027 & $4.863019 \quad-0.297987$ \\
\hline 1 & 0 & -2.050558 & $1.124083 \quad 2.032169$ & 1 & 0 & -2.265533 & $-2.001941 \quad 0.171997$ \\
\hline 1 & 0 & -3.894097 & $1.521361 \quad 0.664395$ & 1 & 0 & -4.383560 & $-1.060873-0.530749$ \\
\hline 8 & 0 & 0.310360 & $2.408122 \quad 1.243003$ & 8 & 0 & -1.198253 & $-1.512084 \quad 2.496175$ \\
\hline 1 & 0 & -2.754507 & $0.951603-1.826527$ & 1 & 0 & -3.856936 & $1.448961 \quad 0.527964$ \\
\hline 1 & 0 & -3.724497 & $-1.547541 \quad 3.610802$ & 1 & 0 & -2.052127 & $-2.919557 \quad-3.299084$ \\
\hline 1 & 0 & -4.559798 & $-0.419731 \quad 2.510754$ & 1 & 0 & -3.491402 & $-2.056880 \quad-2.712010$ \\
\hline 1 & 0 & -3.373568 & $0.201844 \quad 3.704628$ & 1 & 0 & -2.798453 & $-3.397873-1.744825$ \\
\hline 1 & 0 & -1.416041 & $3.055871-1.589036$ & 1 & 0 & -3.844365 & $0.440519 \quad 2.816497$ \\
\hline 1 & 0 & -1.843364 & $3.135993 \quad 0.129088$ & 1 & 0 & -3.699425 & $-1.175103 \quad 2.110449$ \\
\hline 1 & 0 & -5.696926 & $-0.988921-1.059482$ & 1 & 0 & -4.710592 & $1.481966-2.984674$ \\
\hline 1 & 0 & -4.948997 & $0.465680-1.771108$ & 1 & 0 & -5.175155 & $1.597267-1.266001$ \\
\hline 1 & 0 & -5.855276 & $0.595852-0.242274$ & 1 & 0 & -5.562050 & $0.124278-2.190475$ \\
\hline 1 & 0 & -0.627927 & $-1.908635 \quad 1.396035$ & 1 & 0 & -0.657207 & $0.471374-1.890026$ \\
\hline 1 & 0 & -4.448343 & $5.058249-1.499131$ & 1 & 0 & -7.278697 & $-0.691420 \quad 2.466909$ \\
\hline 1 & 0 & -2.730459 & $5.115086-1.987084$ & 1 & 0 & -6.082682 & $-0.150509 \quad 3.679179$ \\
\hline 1 & 0 & -3.167283 & $5.168699-0.257140$ & 1 & 0 & -5.935514 & $-1.770042 \quad 2.945088$ \\
\hline 1 & 0 & -2.666346 & $-2.813309 \quad 0.369595$ & 1 & 0 & -0.236066 & $2.073455 \quad 1.551774$ \\
\hline 1 & 0 & 0.770578 & $-1.844477-1.999432$ & 1 & 0 & -0.052987 & $2.521607-2.725222$ \\
\hline 1 & 0 & 5.759504 & $-0.023745 \quad 1.386121$ & 1 & 0 & 5.386192 & $-2.705951-0.416658$ \\
\hline 1 & 0 & 4.380558 & $1.229441-3.249740$ & 1 & 0 & 5.682952 & $1.802791 \quad 1.723565$ \\
\hline 1 & 0 & -3.563761 & $-4.200043-1.478725$ & 1 & 0 & 0.257370 & $4.476905 \quad 1.821017$ \\
\hline 1 & 0 & -0.135160 & $-3.200433-3.877380$ & 1 & 0 & 0.414316 & $4.939568 \quad-2.455407$ \\
\hline 1 & 0 & 7.470090 & $0.354094-0.378500$ & 1 & 0 & 7.442978 & $-2.021643 \quad 0.808270$ \\
\hline 1 & 0 & 6.788940 & $0.978251 \quad-2.682473$ & 1 & 0 & 7.589184 & $0.215680 \quad 1.869603$ \\
\hline 1 & 0 & -2.304855 & $-4.390806-3.617314$ & 1 & 0 & 0.572828 & $5.922293-0.174720$ \\
\hline
\end{tabular}




\section{References}

1. Burla, M. C.; Caliandro, R.; Carrozzini, B.; Cascarano, G. L.; Cuocci, C.; Giacovazzo, C.; Mallamo, M.; Mazzone, A.; Polidori, G. J. Appl. Cryst. 2015, 48, 306.

2. Sheldrick, G. M. Acta. Crystallogr. A 2008, 64, 112.

3. Yadokari-XG; Wakita, K; Kabuto, C.; Akine, S.; Nemoto, T.; Kwon, E. Software for Crystal Structure Analyses, Release of Software (Yadokari-XG 2009) for Crystal Structure Analyses, (2001) J. Cryst. Soc. Jpn. 2009, 51, 218

4. Frisch, M. J.; Trucks, G. W.; Schlegel, H. B.; Scuseria, G. E.; Robb, M. A.; Cheeseman, J. R.; Scalmani, G.; Barone, V.; Mennucci, B.; Petersson, G. A.; Nakatsuji, H.; Caricato, M.; Li, X.; Hratchian, H. P.; Izmaylov, A. F.; Bloino, J.; Zheng, G.; Sonnenberg, J. L.; Hada, M.; Ehara, M.; Toyota, K.; Fukuda, R.; Hasegawa, J.; Ishida, M.; Nakajima, T.; Honda, Y.; Kitao, O.; Nakai, H.; Vreven, T.; Montgomery, J. A., Jr.; Peralta, J. E.; Ogliaro, F.; Bearpark, M.; Heyd, J. J.; Brothers, E.; Kudin, K. N.; Staroverov, V. N.; Keith, T.; Kobayashi, R.; Normand, J.; Raghavachari, K.; Rendell, A.; Burant, J. C.; Iyengar, S. S.; Tomasi, J.; Cossi, M.; Rega, N.; Millam, J. M.; Klene, M.; Knox, J. E.; Cross, J. B.; Bakken, V.; Adamo, C.; Jaramillo, J.; Gomperts, R.; Stratmann, R. E.; Yazyev, O.; Austin, A. J.; Cammi, R.; Pomelli, C.; Ochterski, J. W.; Martin, R. L.; Morokuma, K.; Zakrzewski, V. G.; Voth, G. A.; Salvador, P.; Dannenberg, J. J.; Dapprich, S.; Daniels, A. D.; Farkas, Ö.; Foresman, J. B.; Ortiz, J. V.; Cioslowski, J.; Fox, D. J. Gaussian, Inc., Wallingford CT, 2010.

5. (a) Fukui, K. Acc. Chem. Res. 1981, 14, 363. (b) Gonzalez, C.; Schlegel, H. B. J. Chem. Phys. 1989, 90, 2154.

(c) Gonzalez, C.; Schlegel, H. B. J. Chem. Phys. 1990, 94, 5523. 


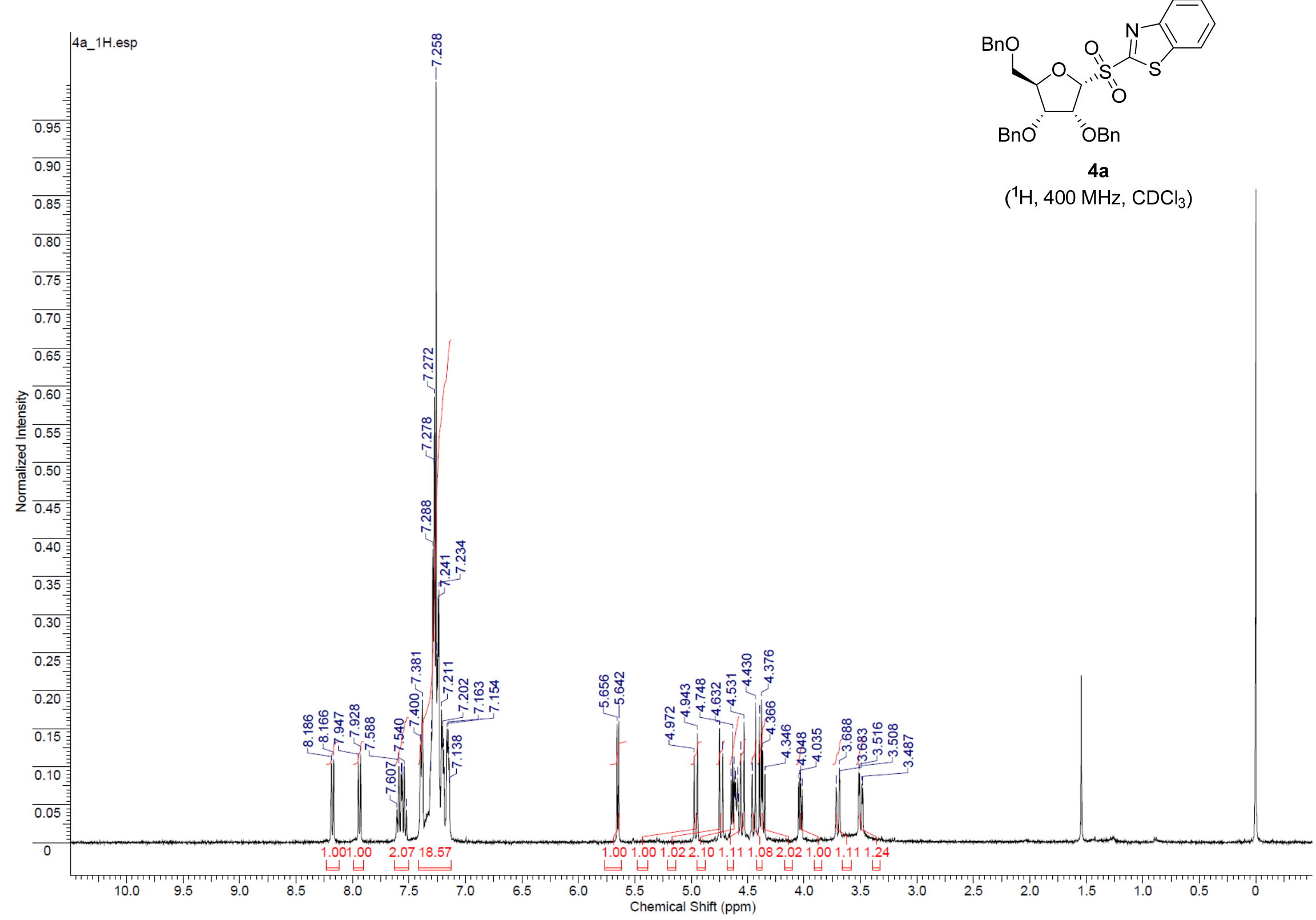




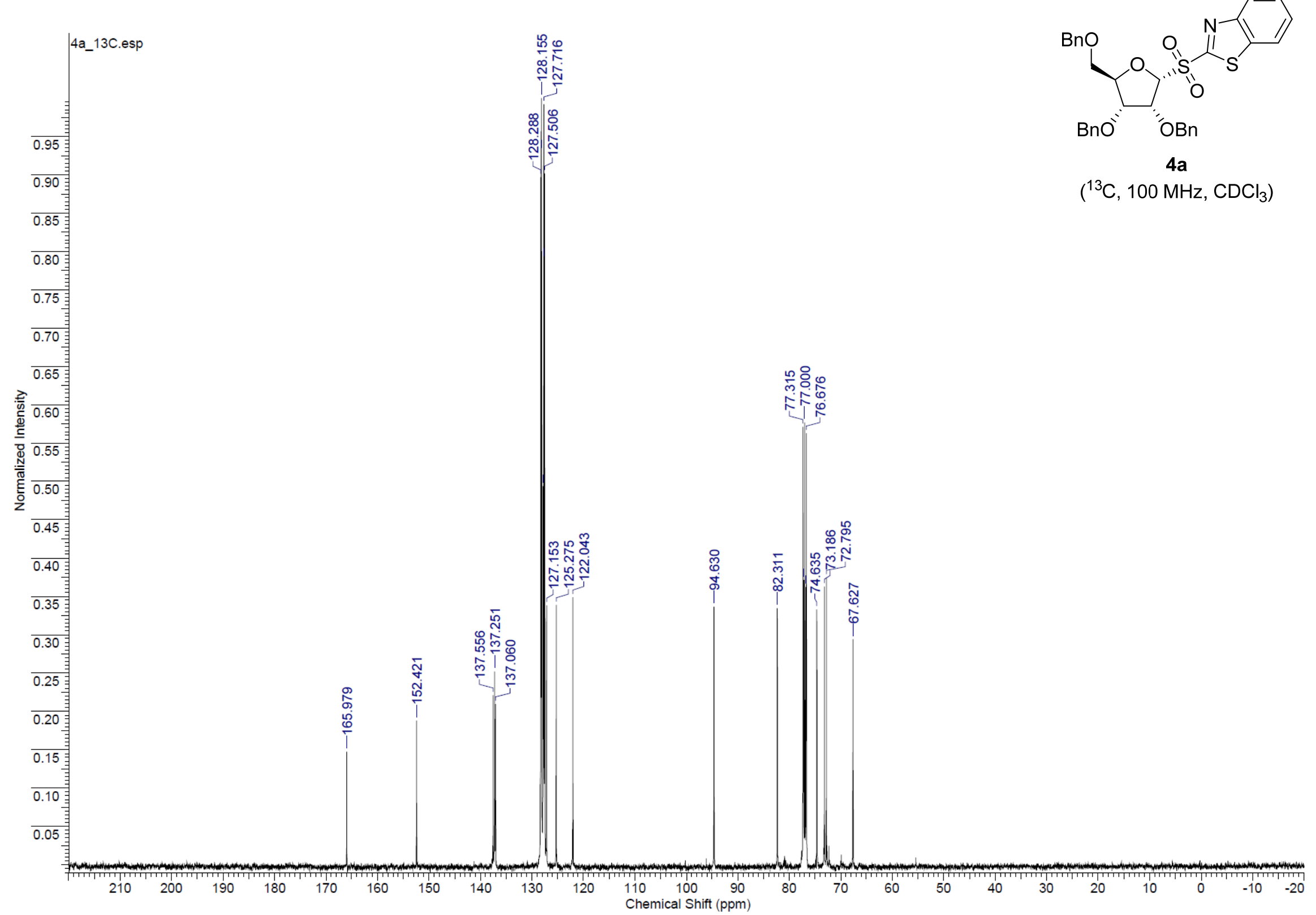




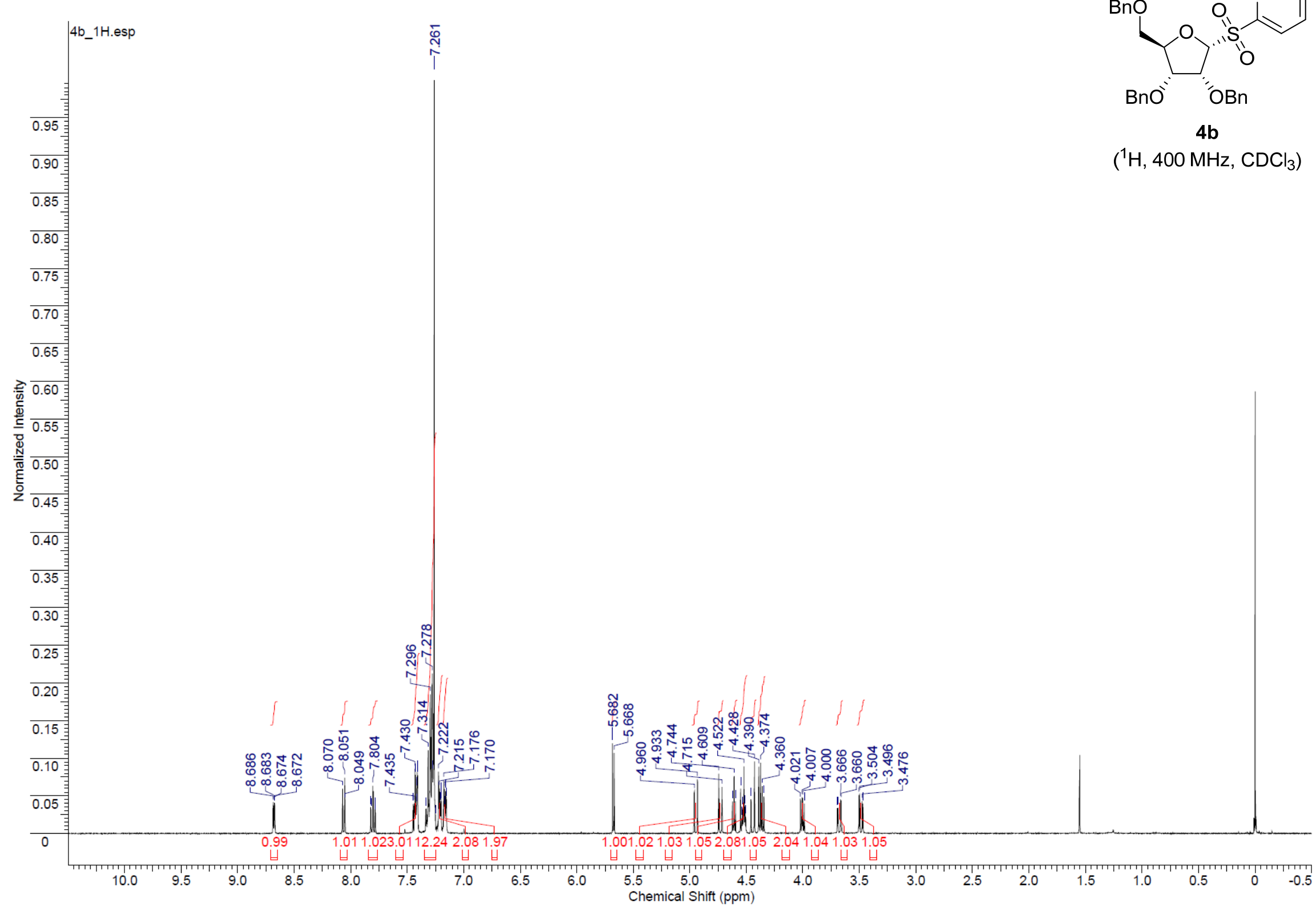




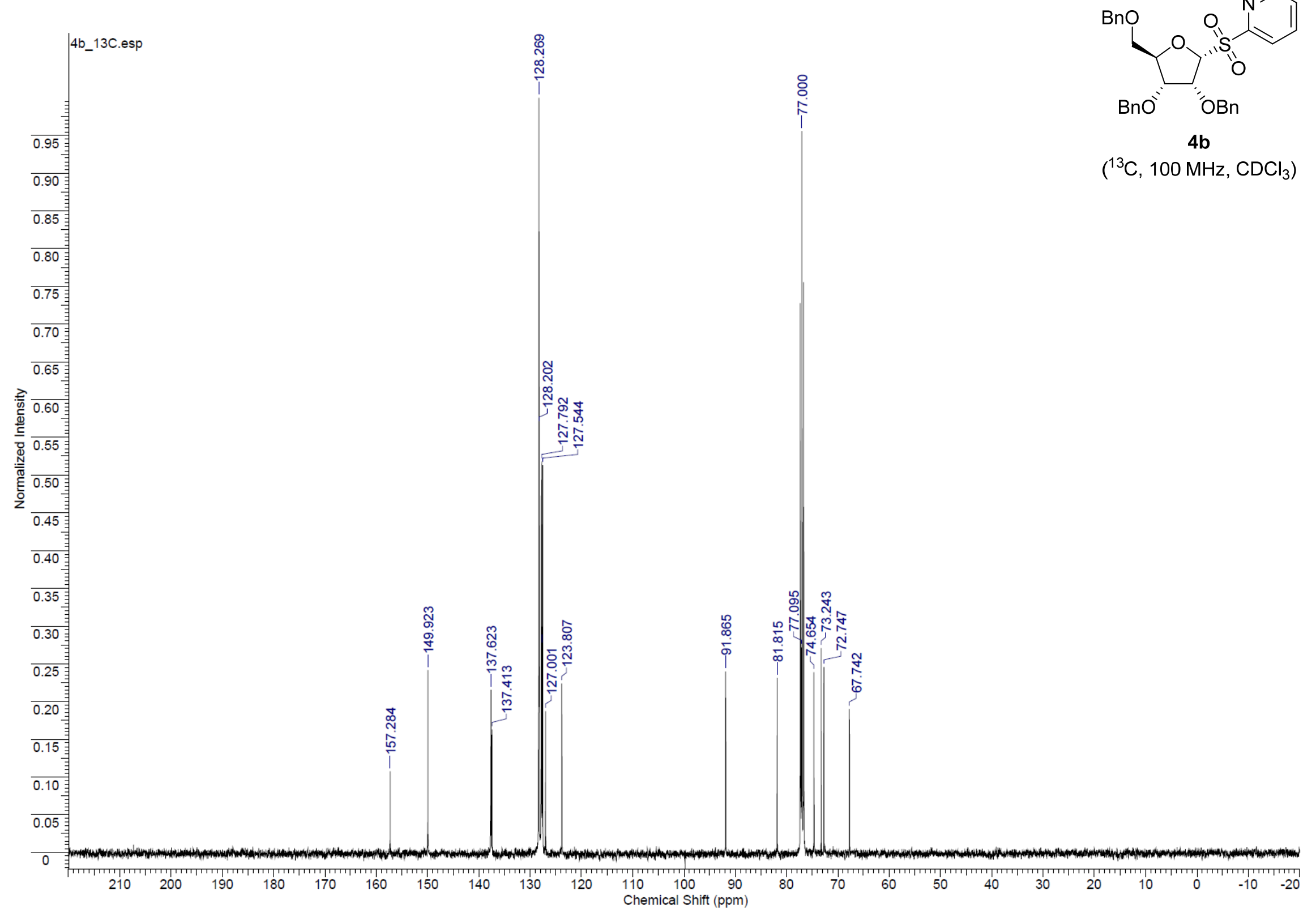




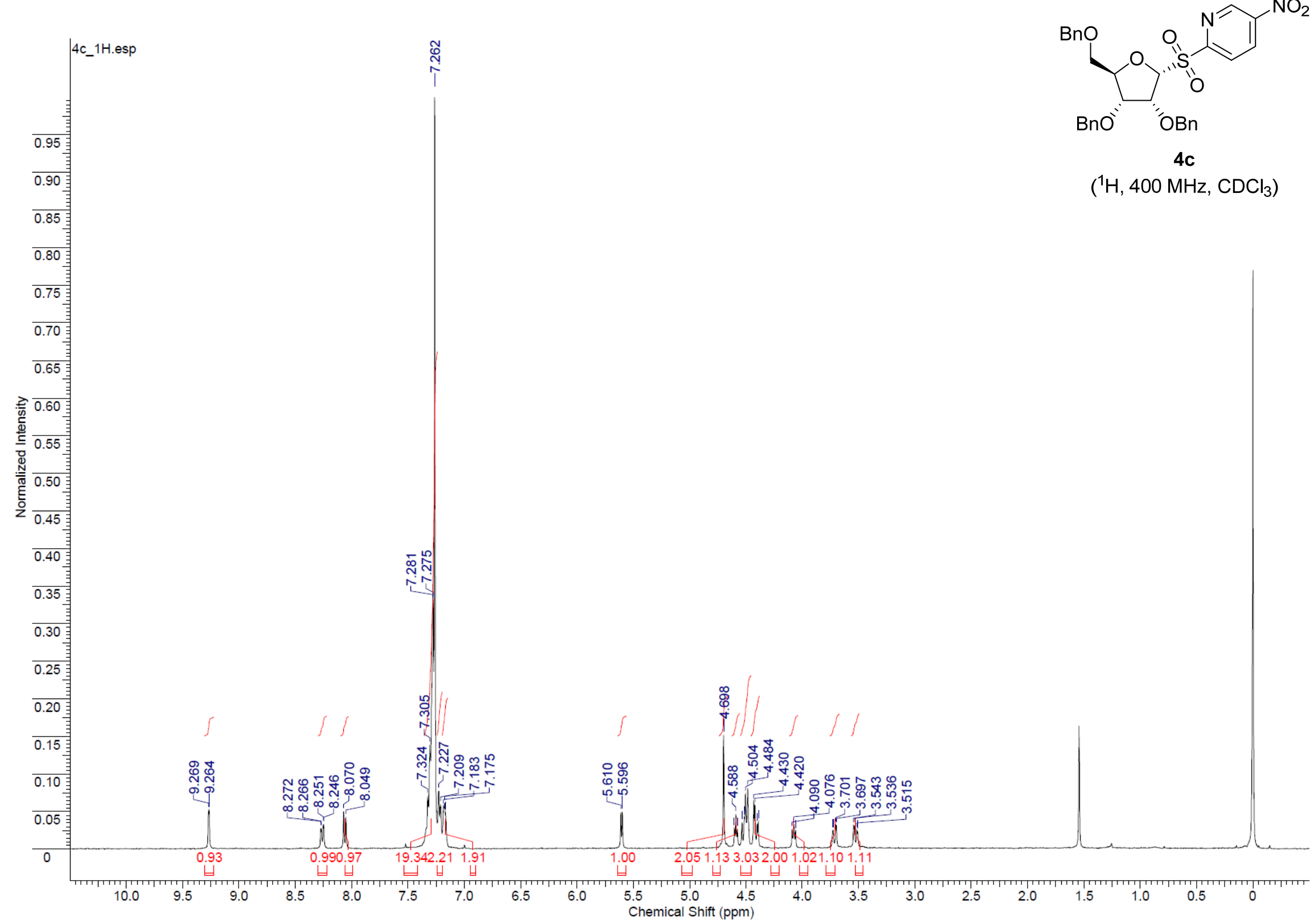




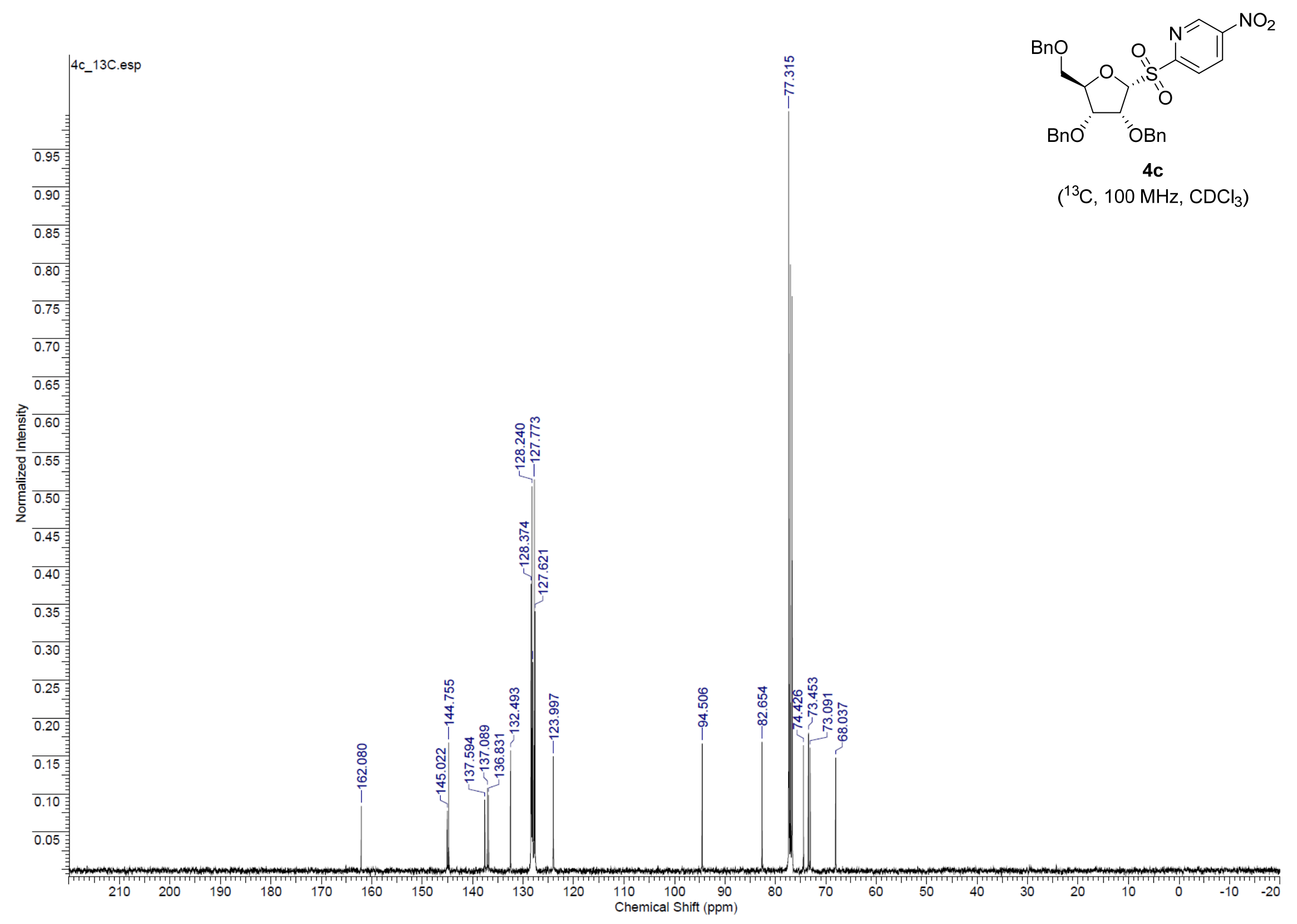




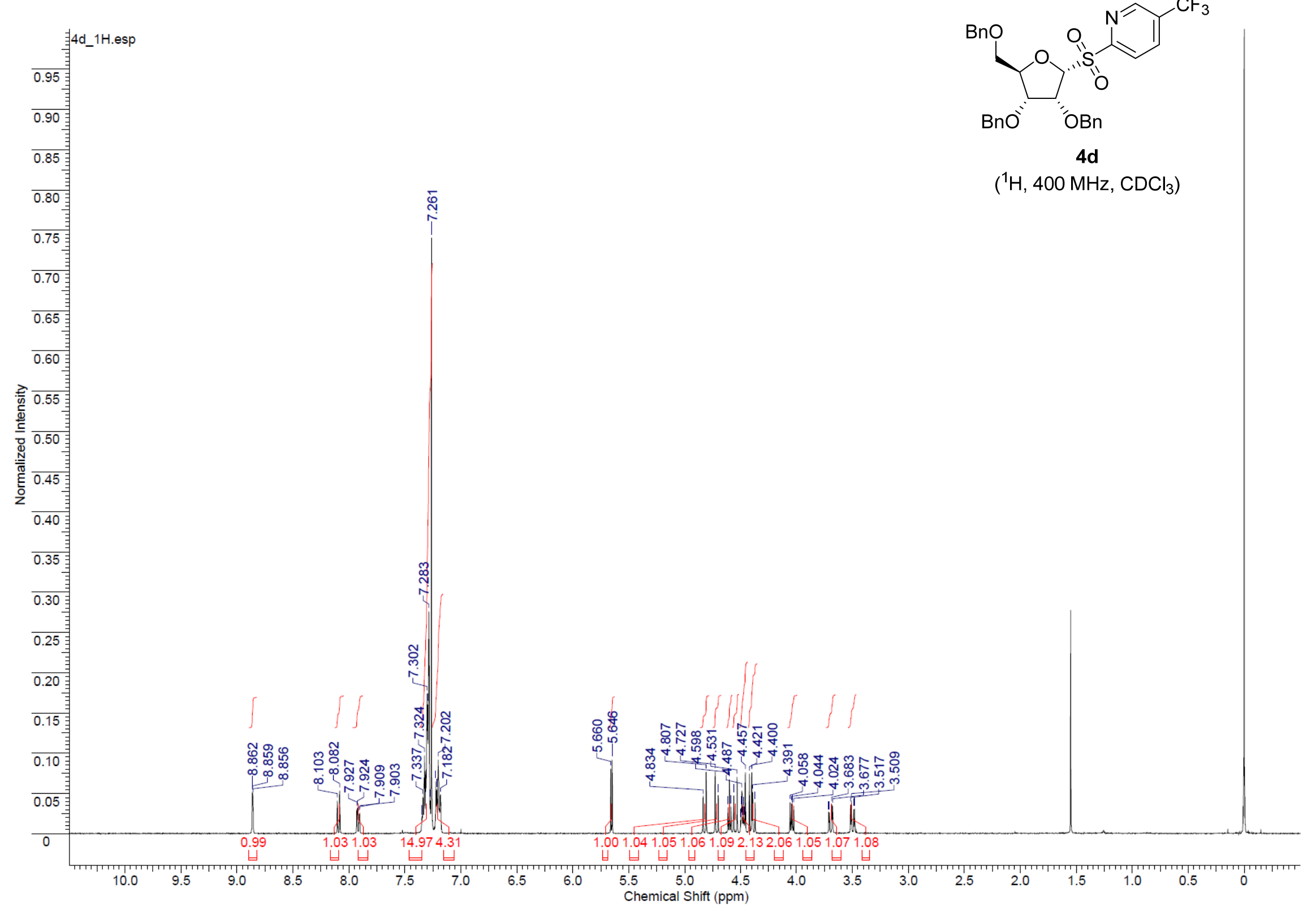




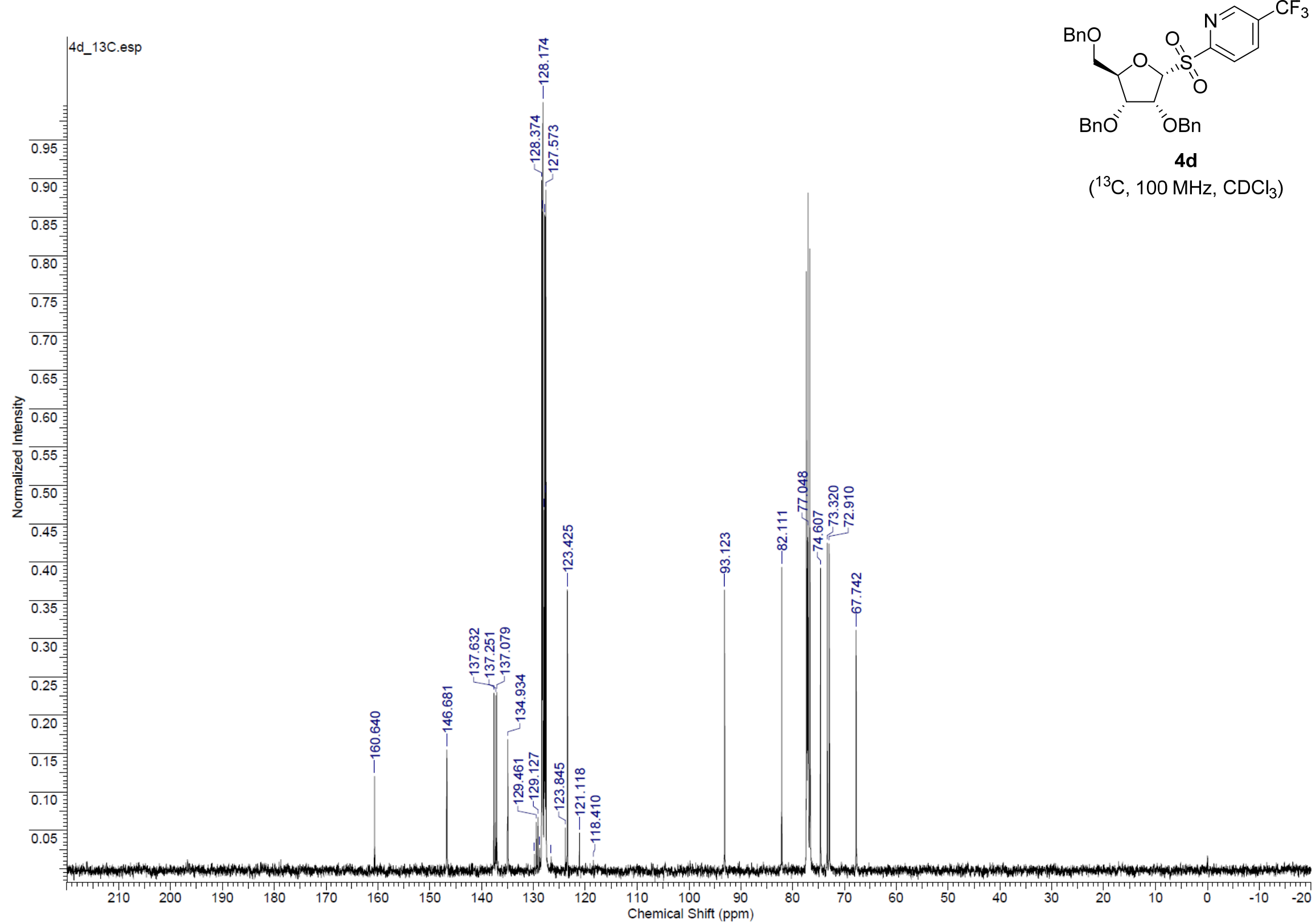




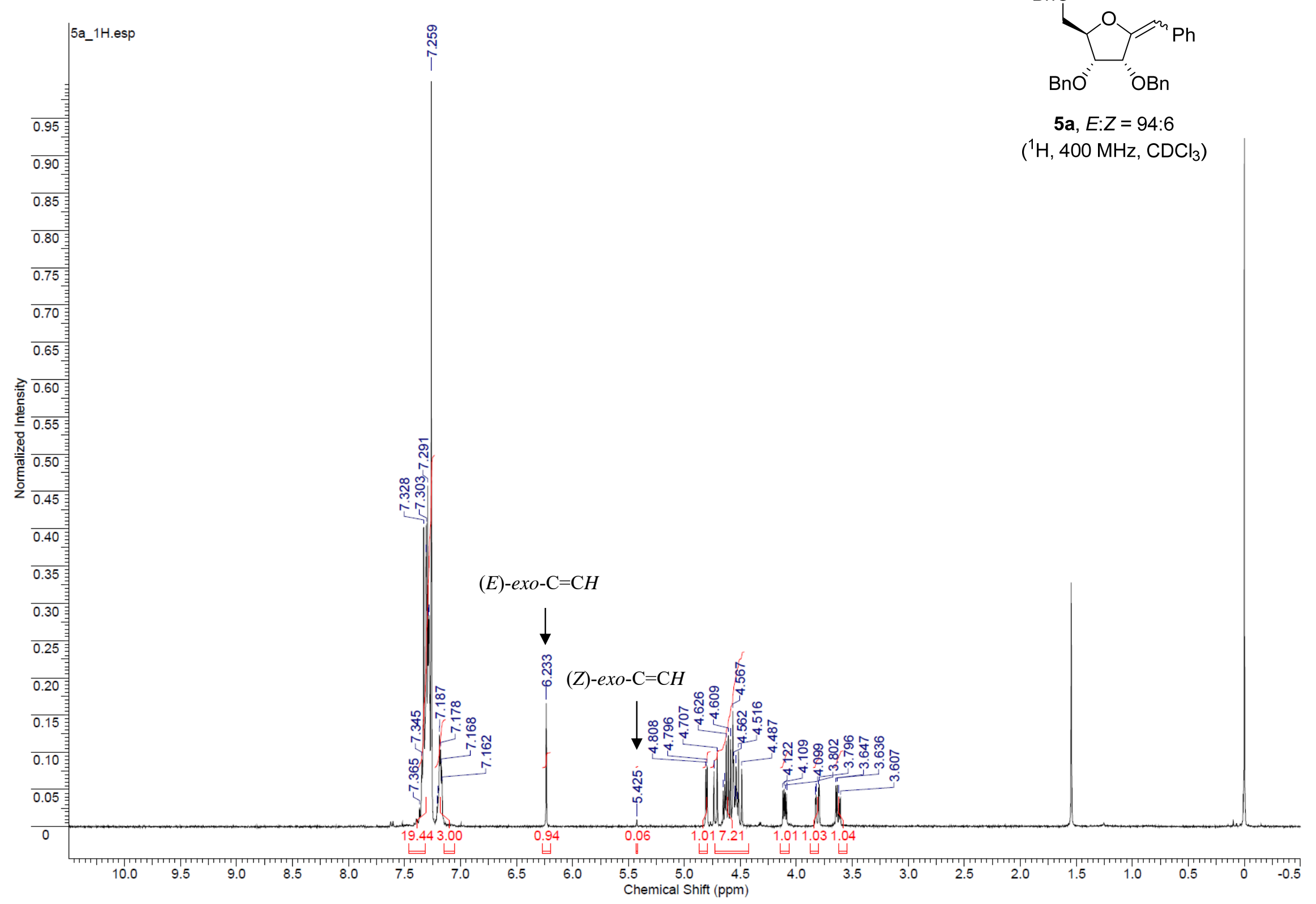




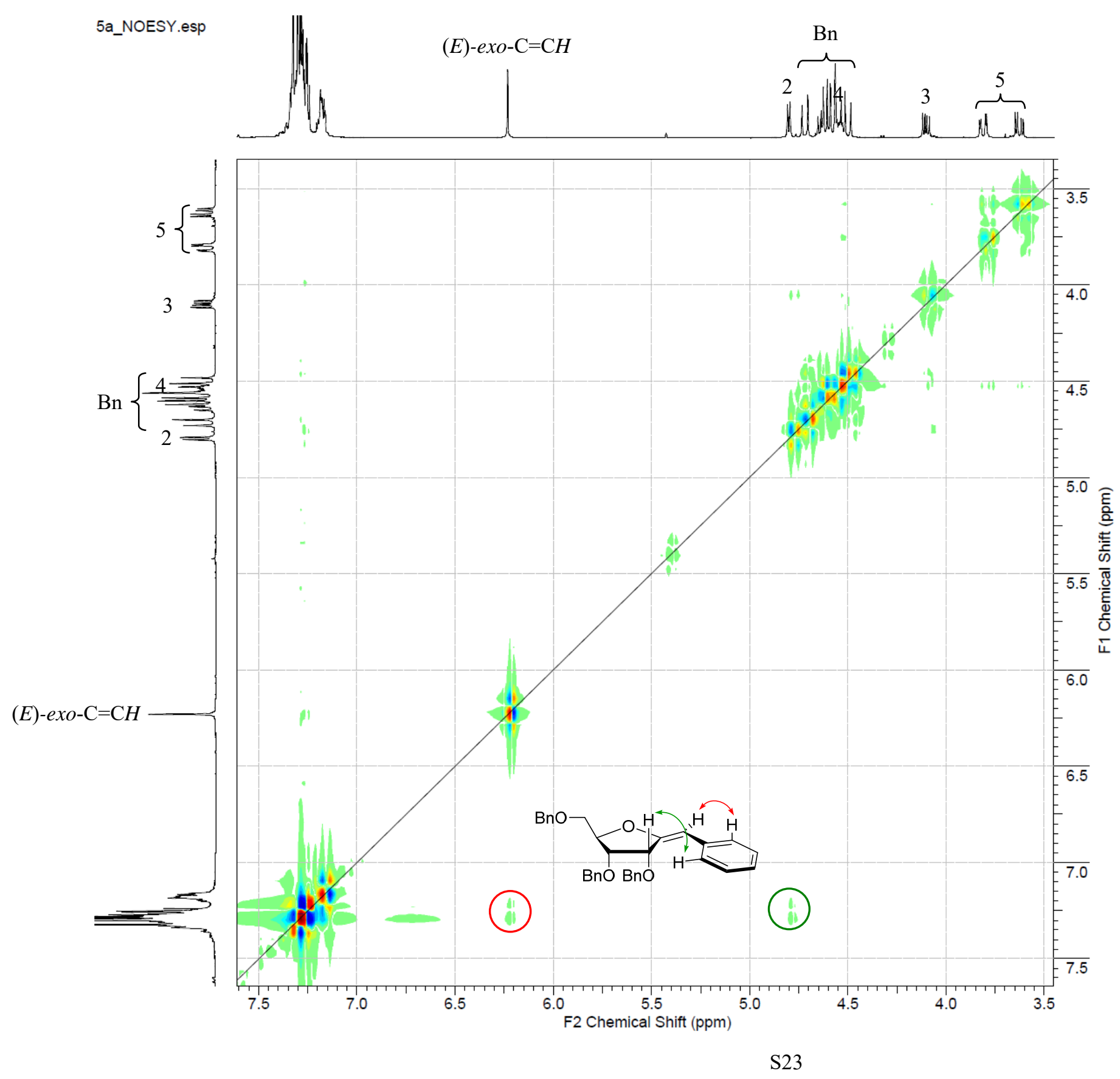

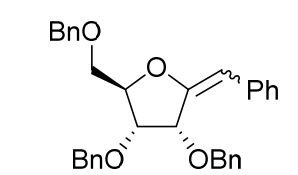

5a, $E: Z=94: 6$ (NOESY, $400 \mathrm{MHz}, \mathrm{CDCl}_{3}$ ) 


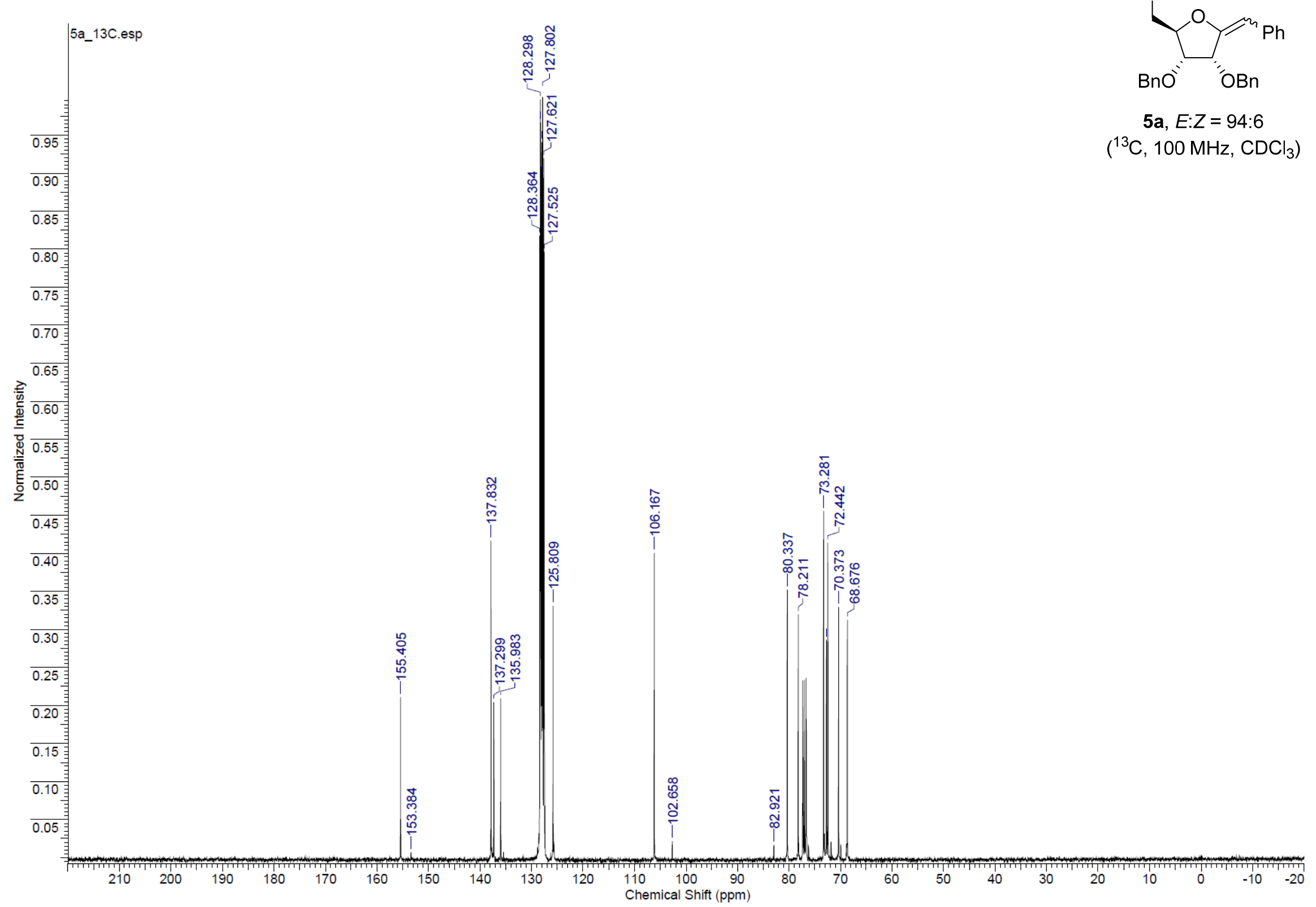




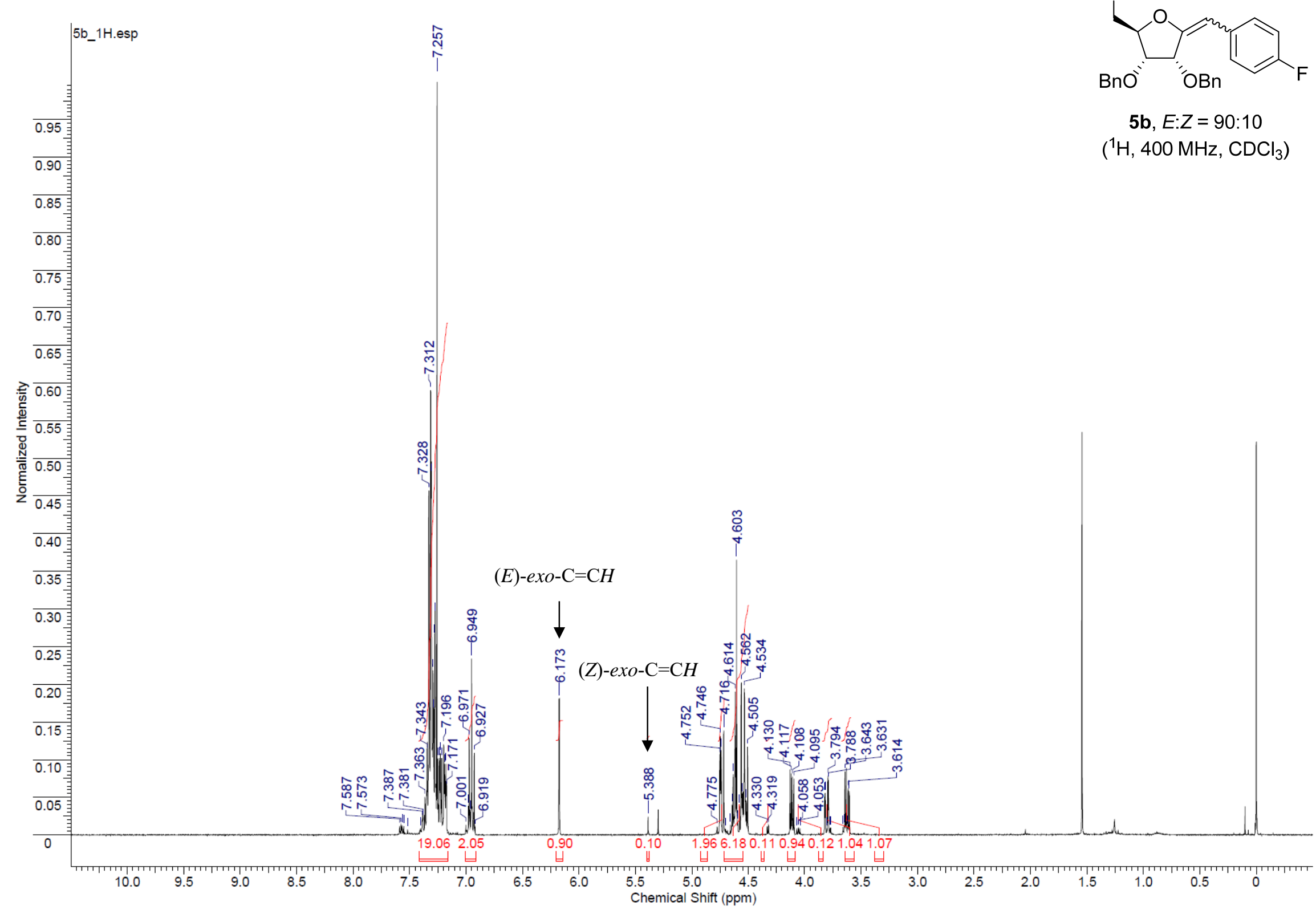




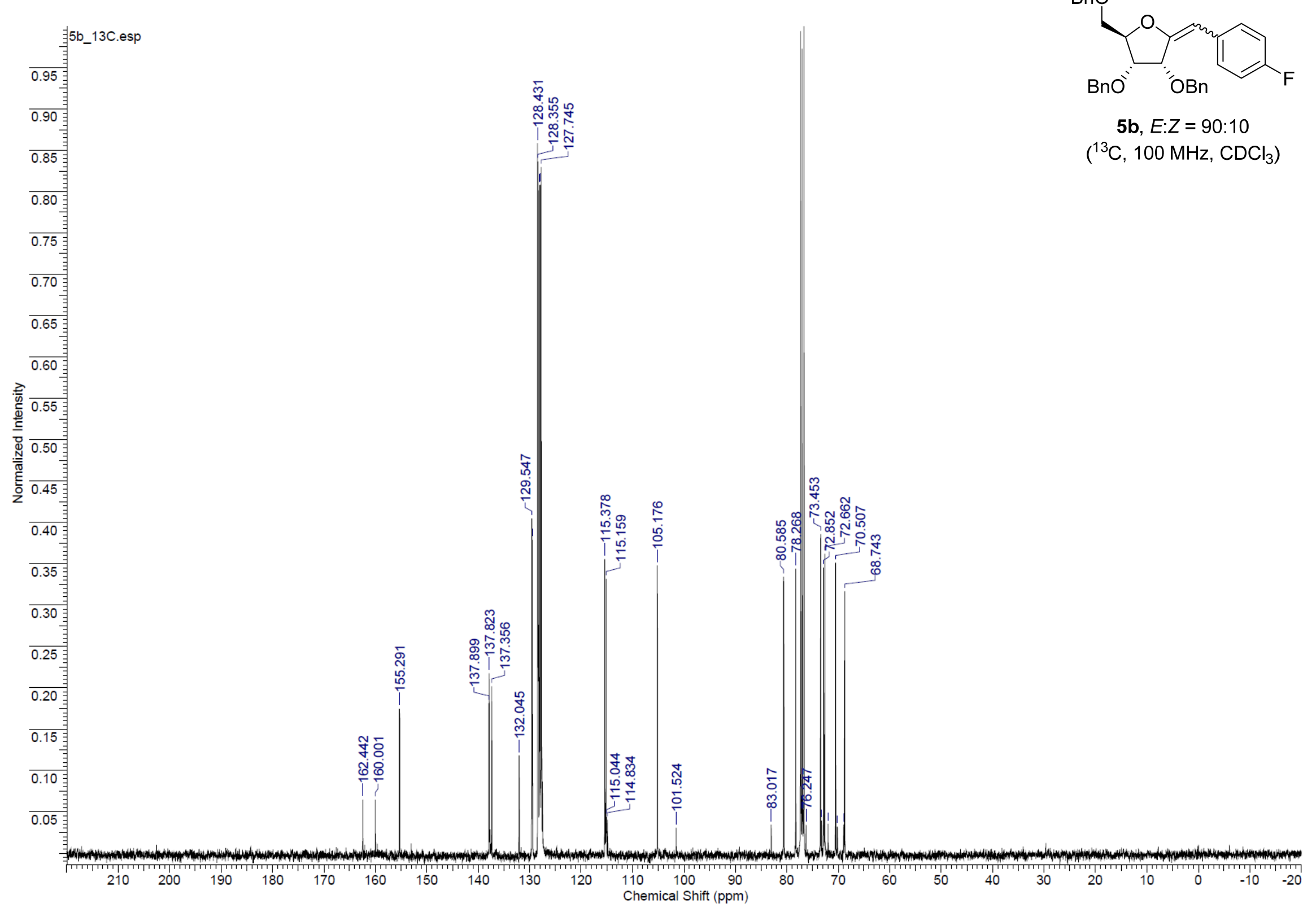




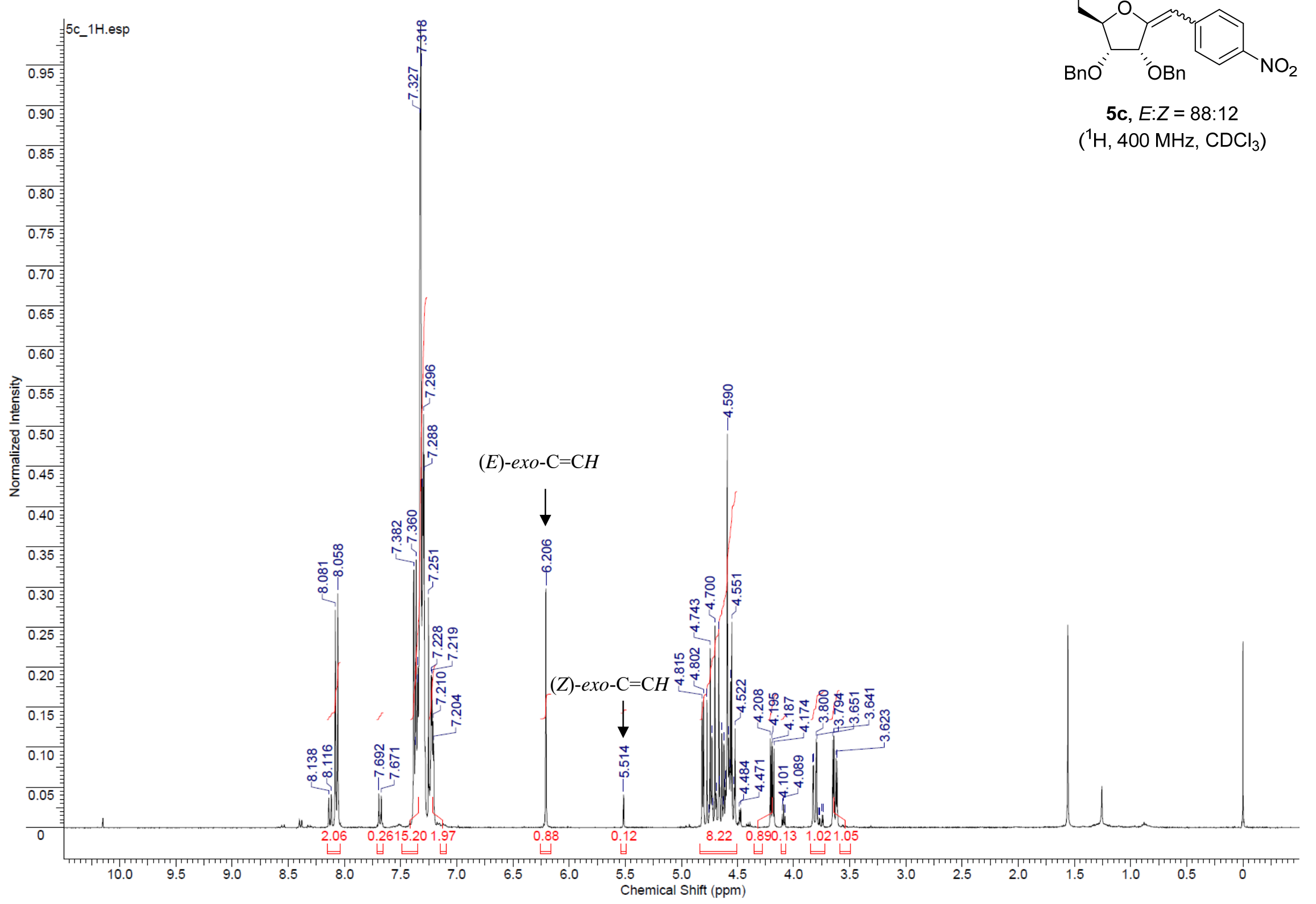




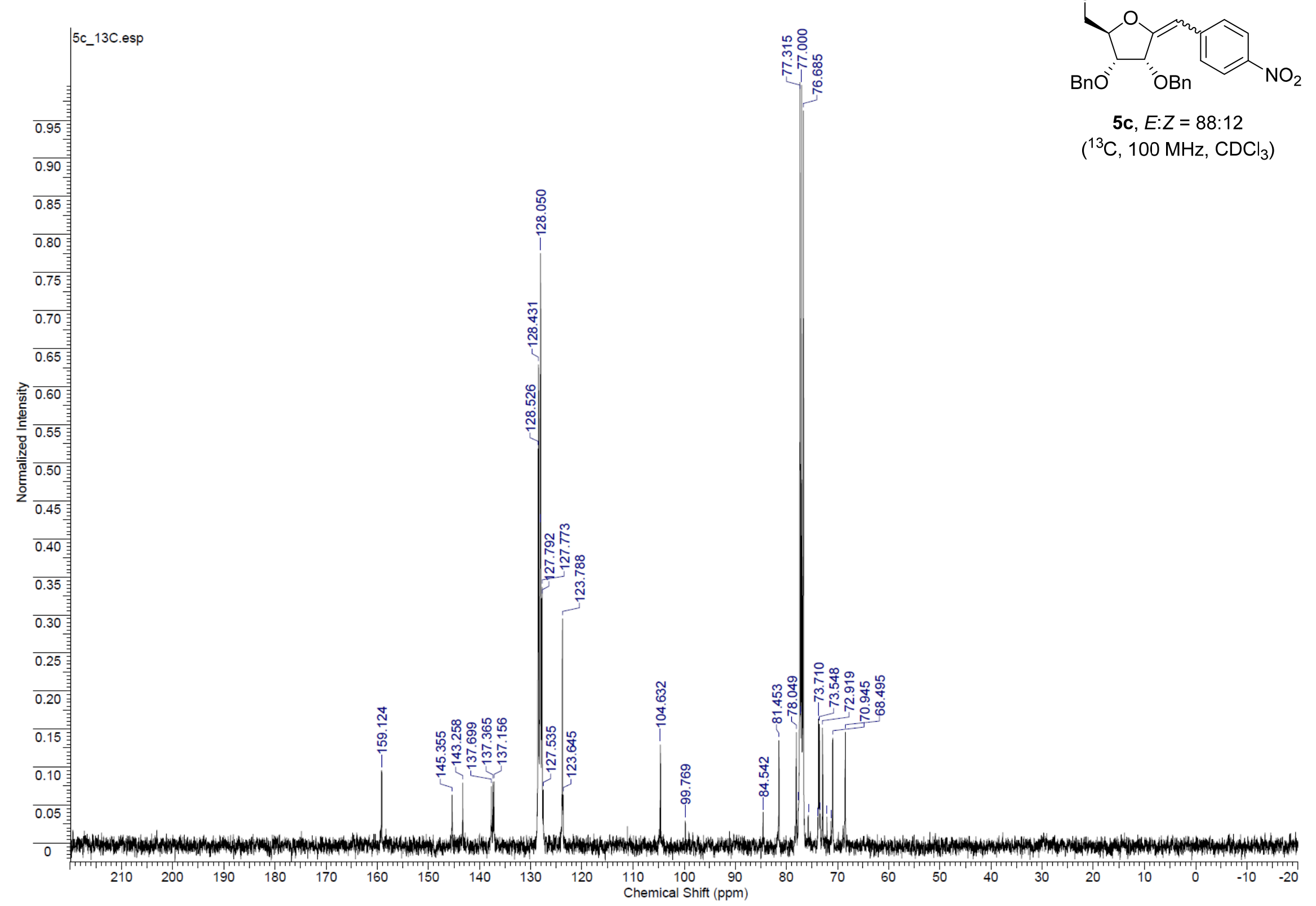




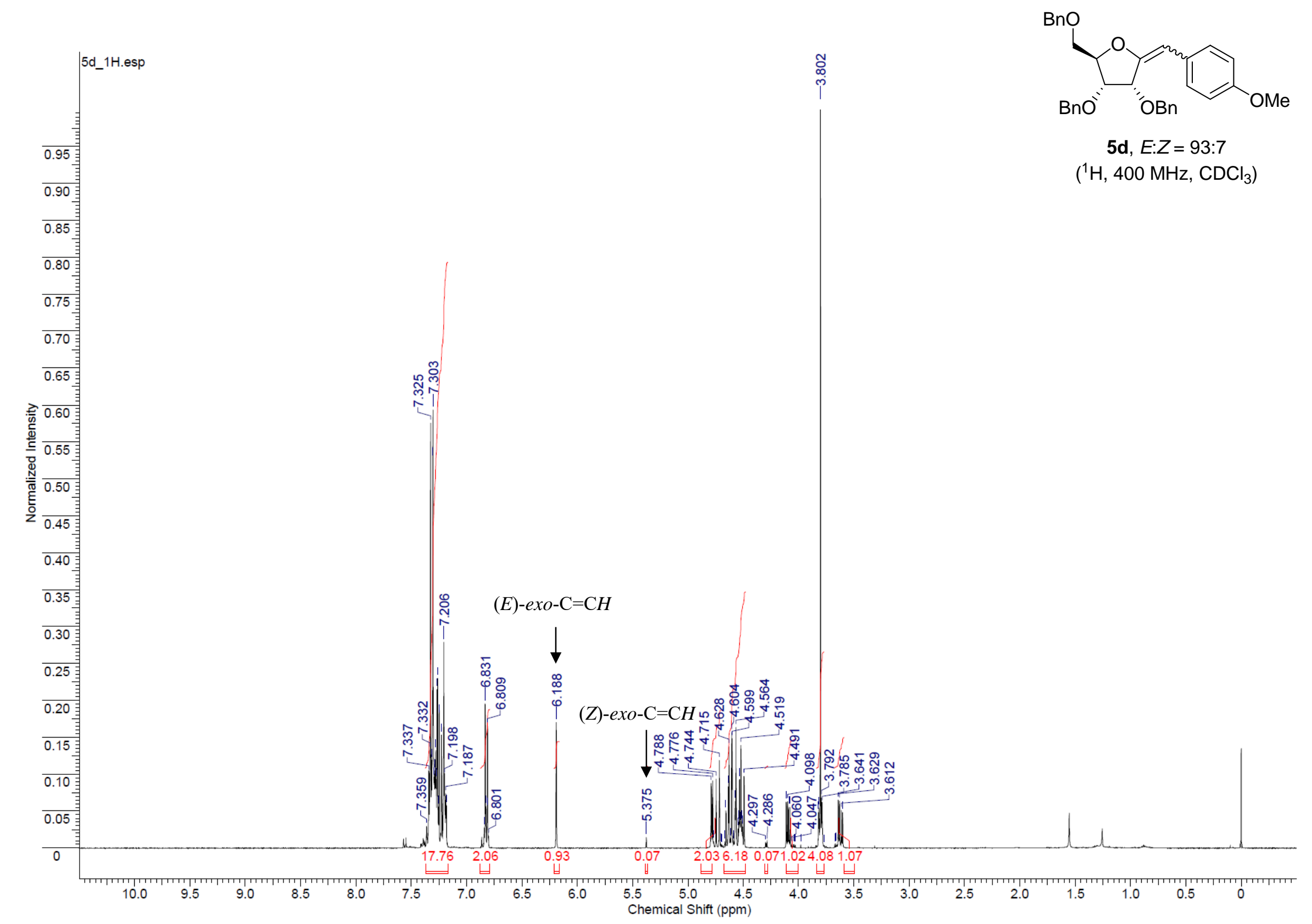




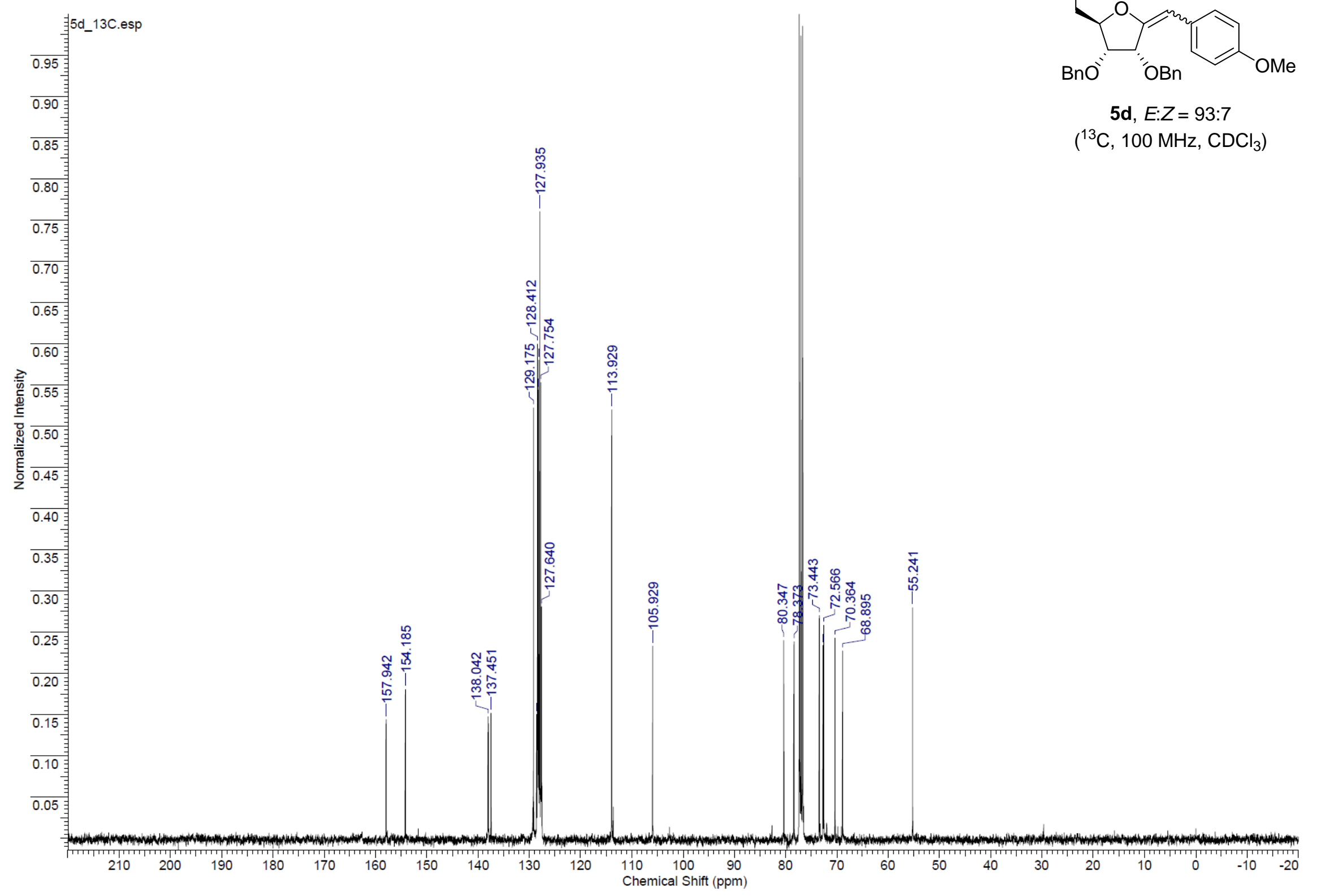




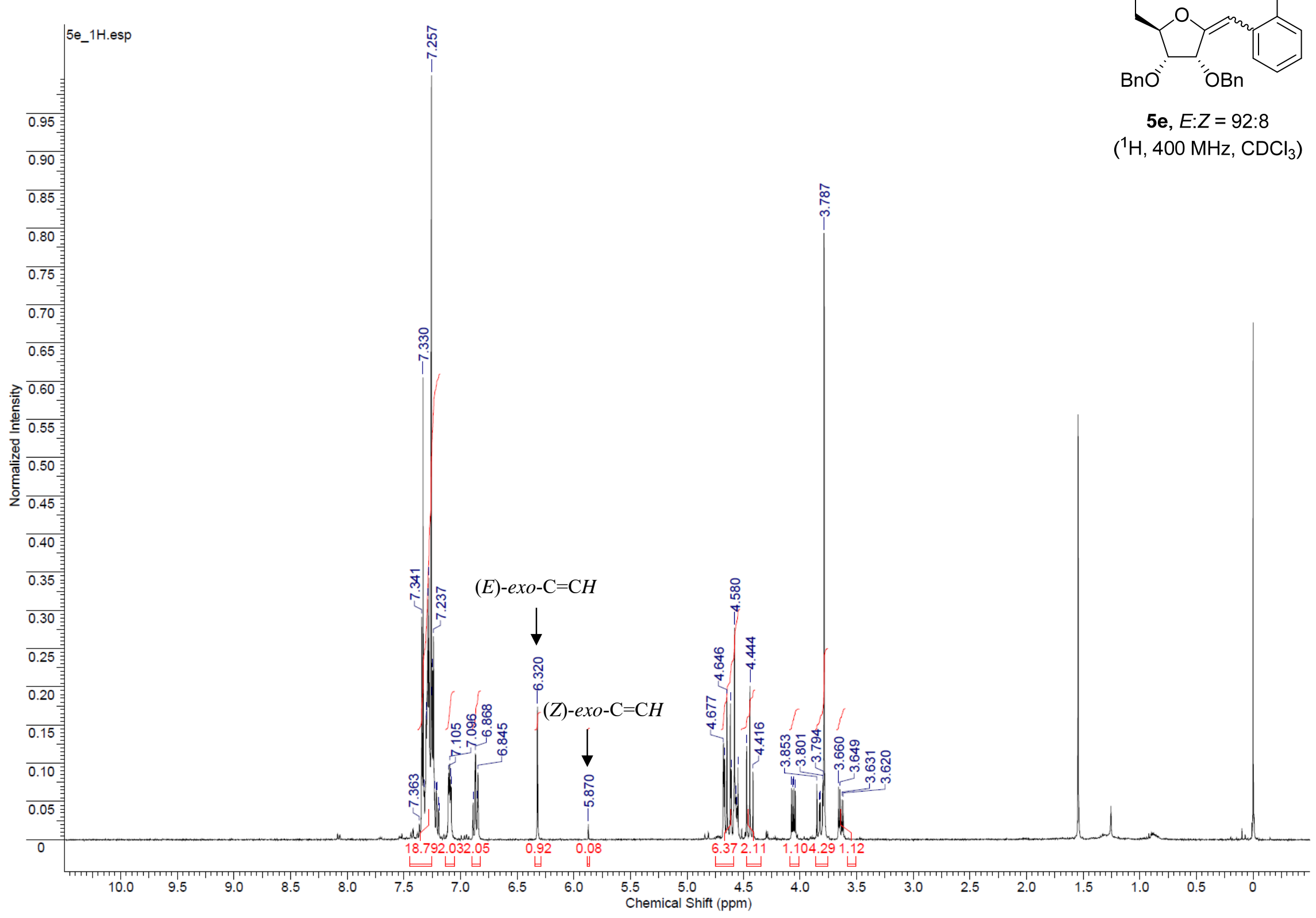




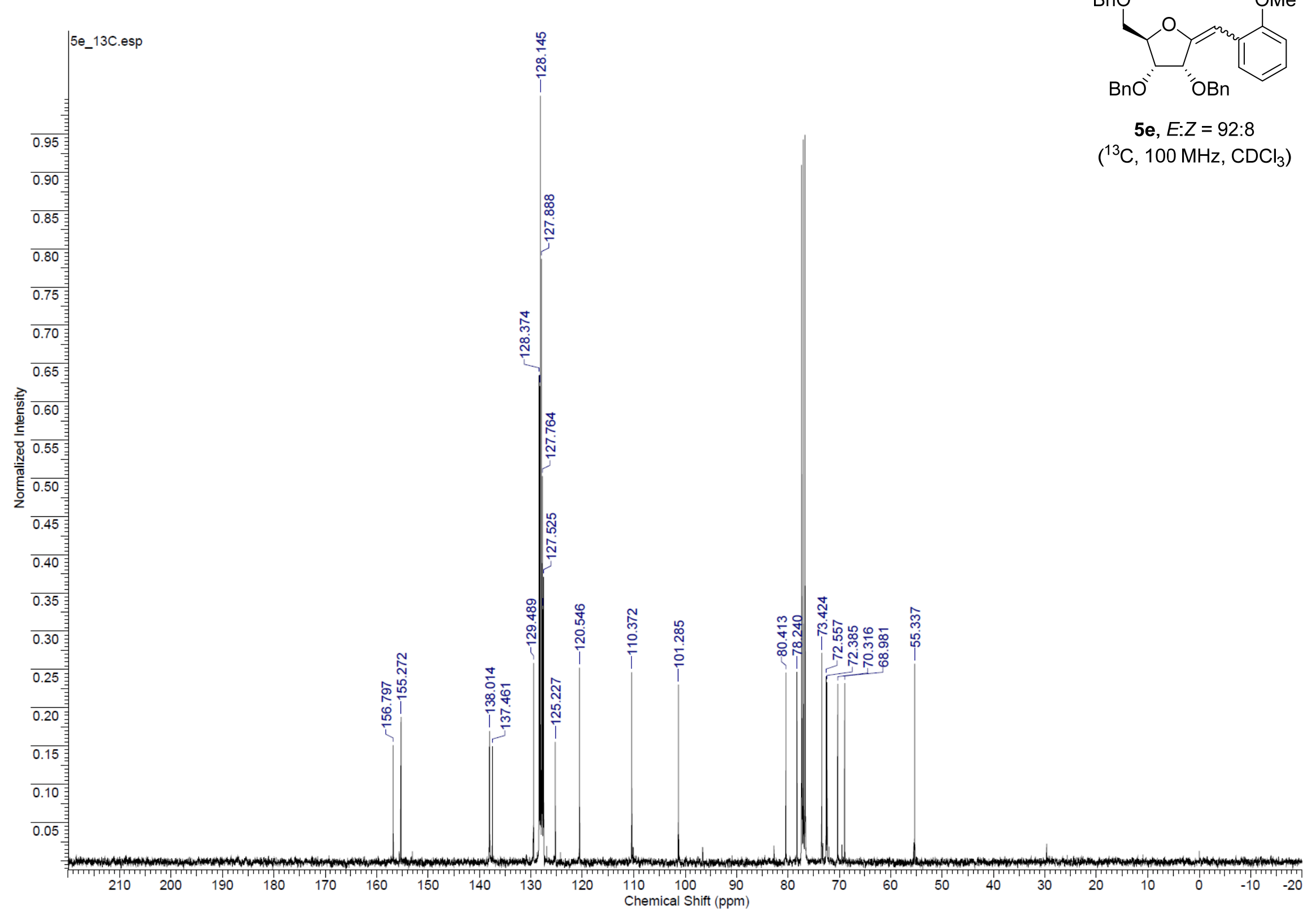




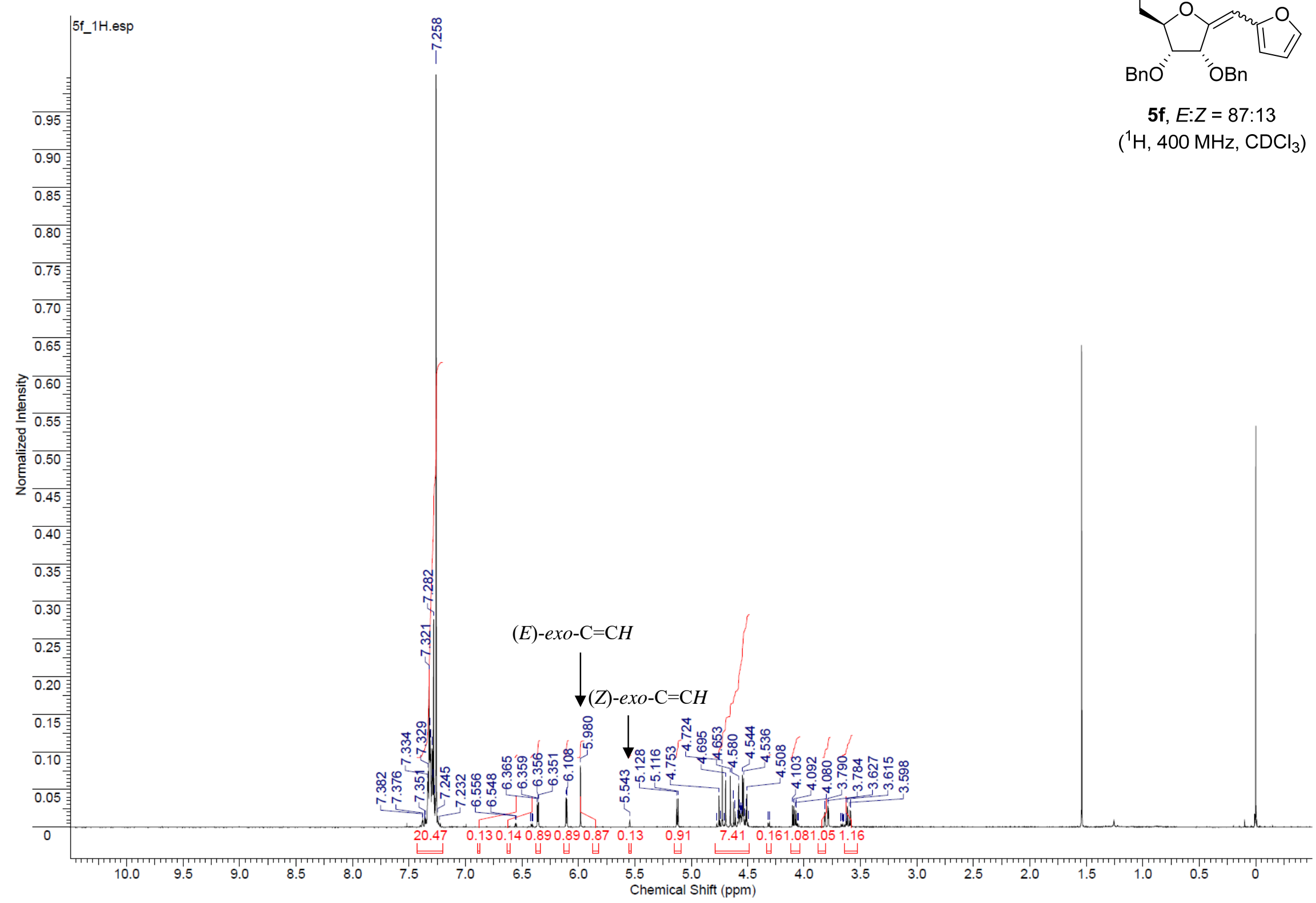




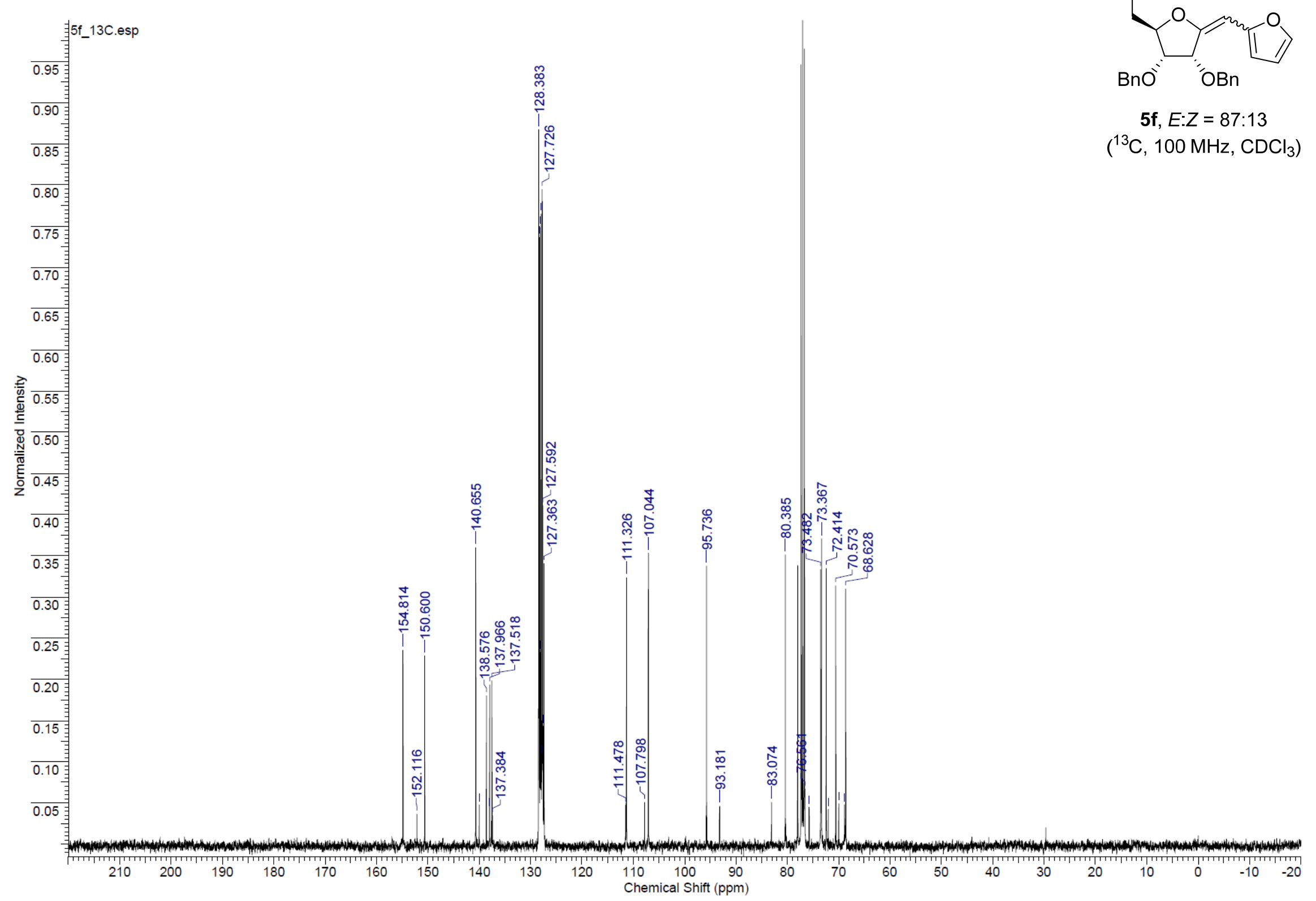




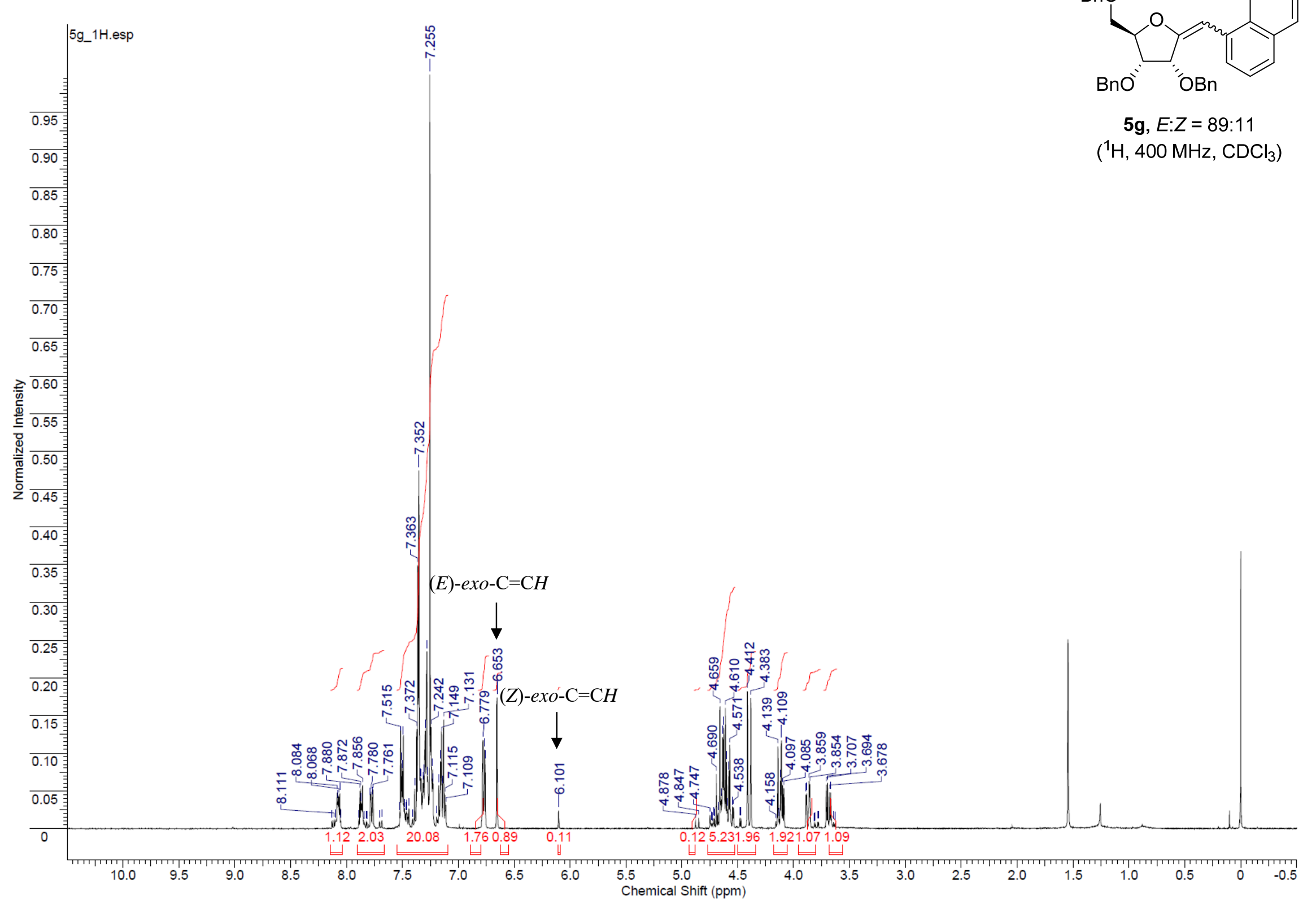




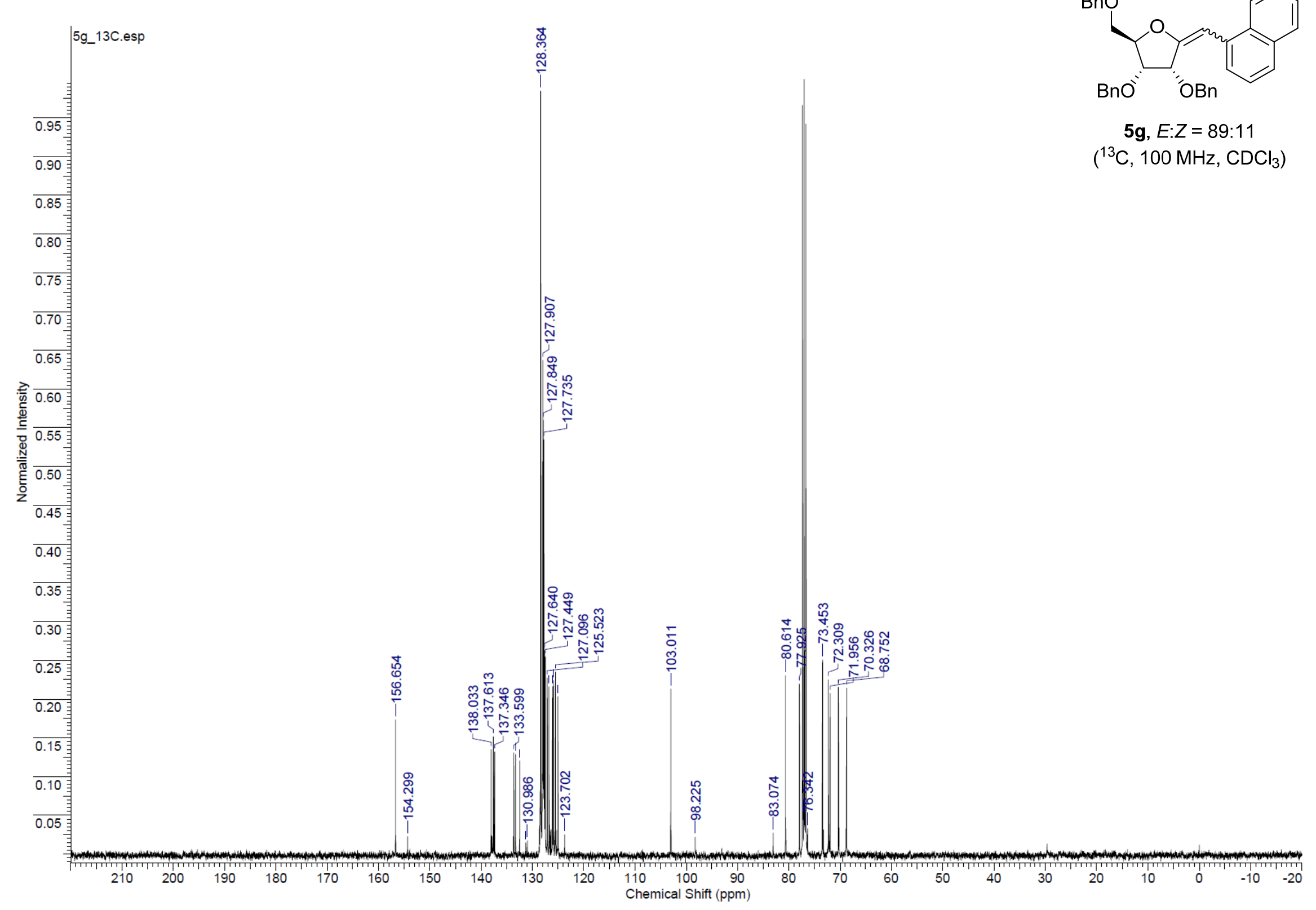




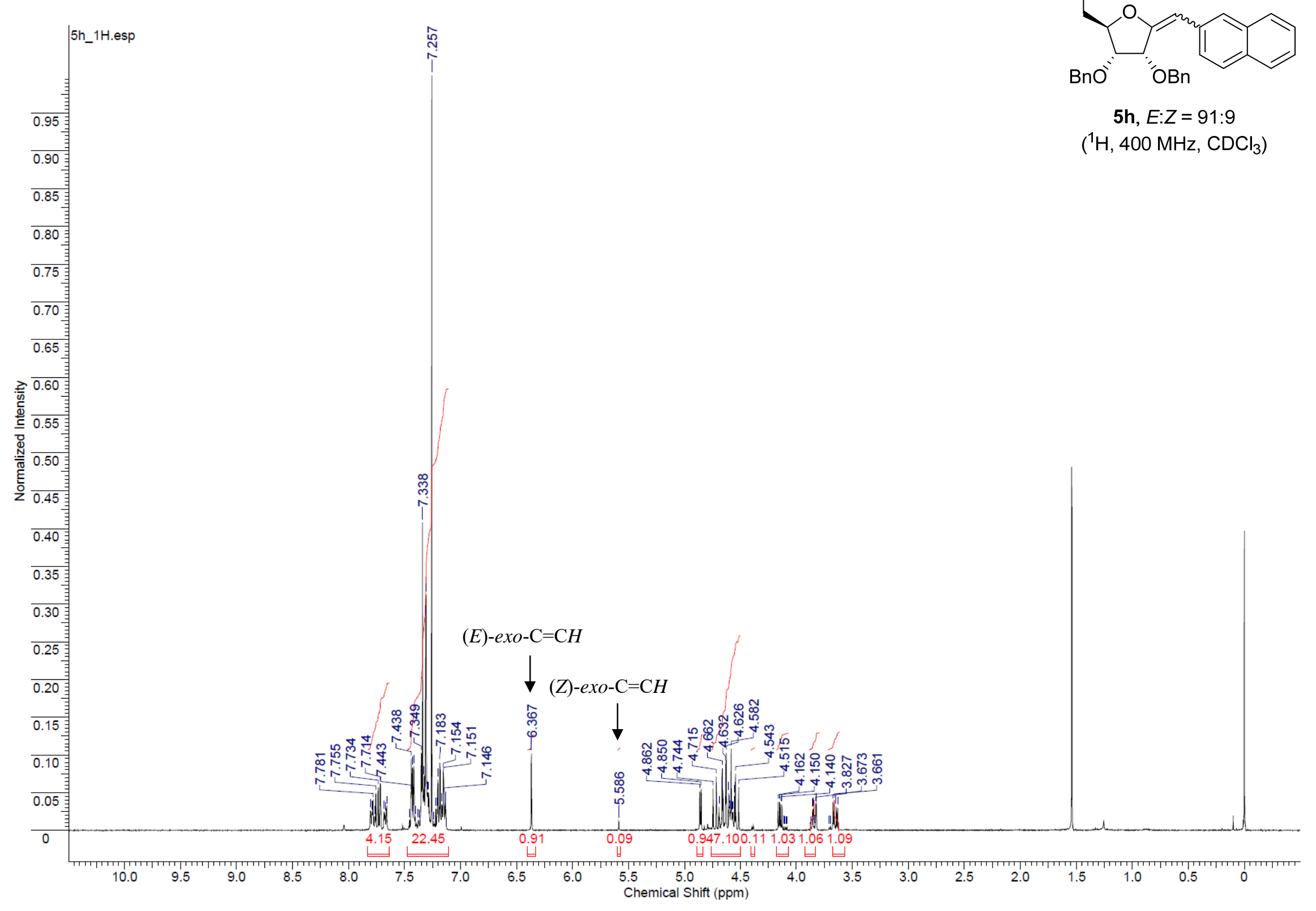




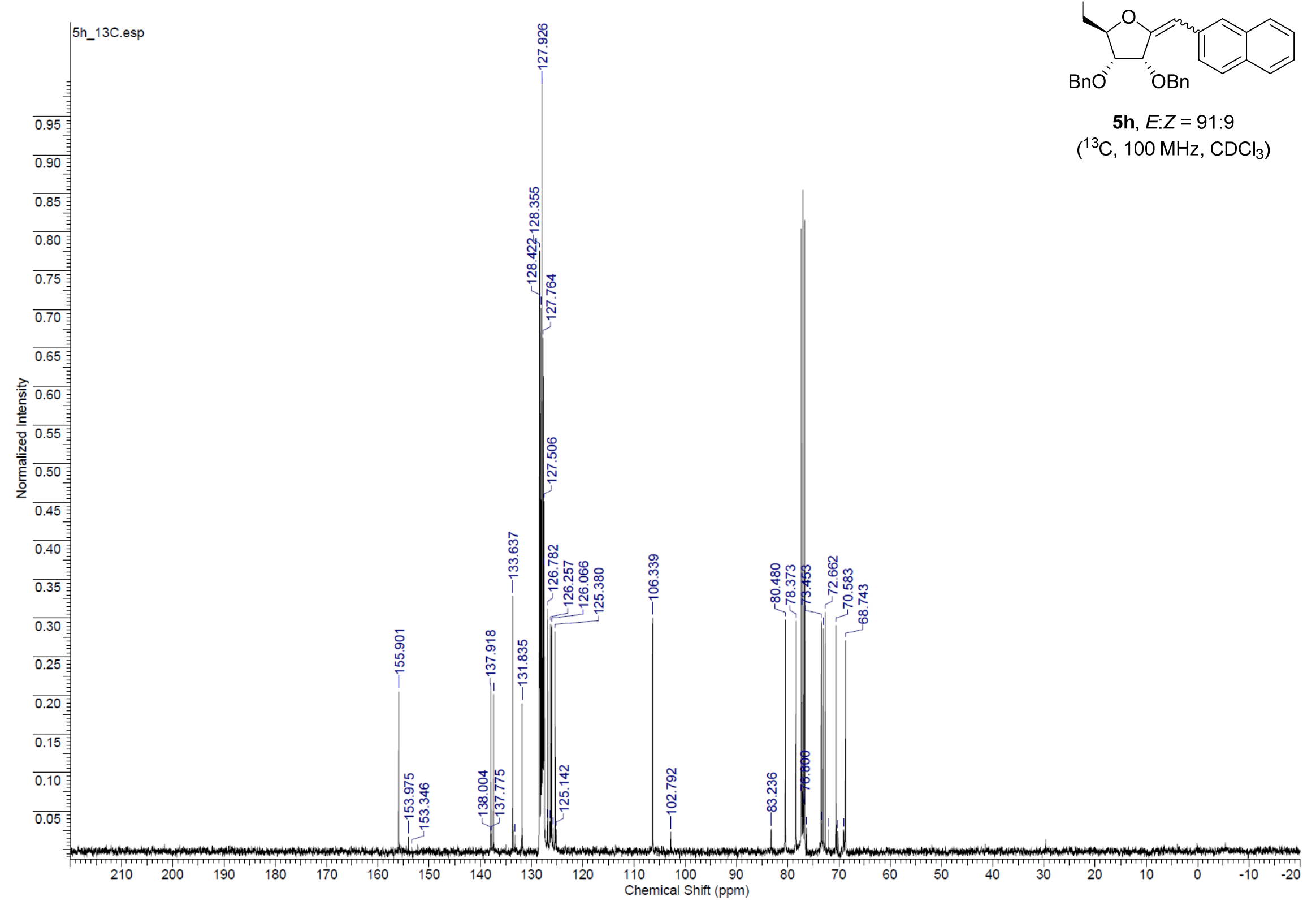




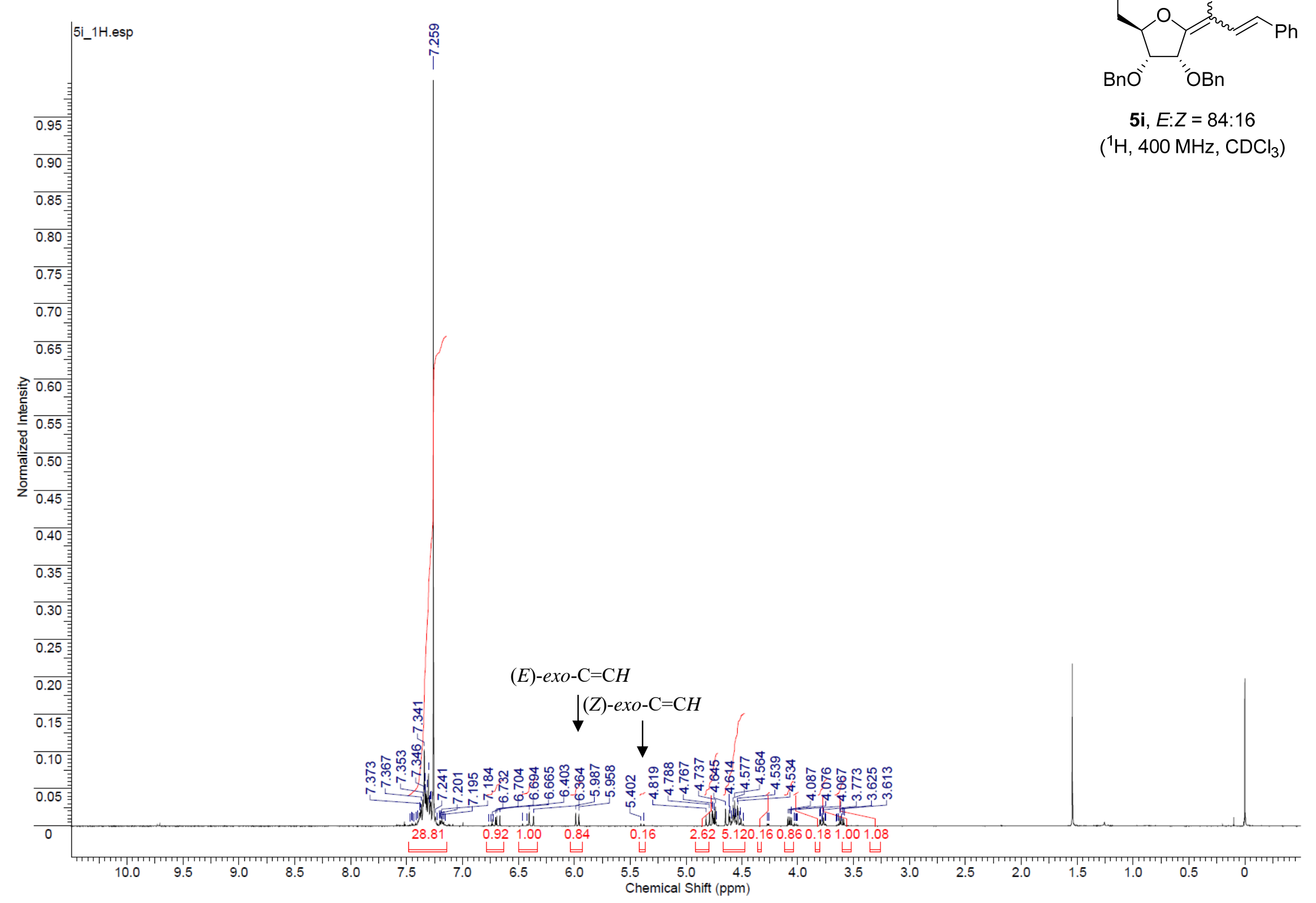




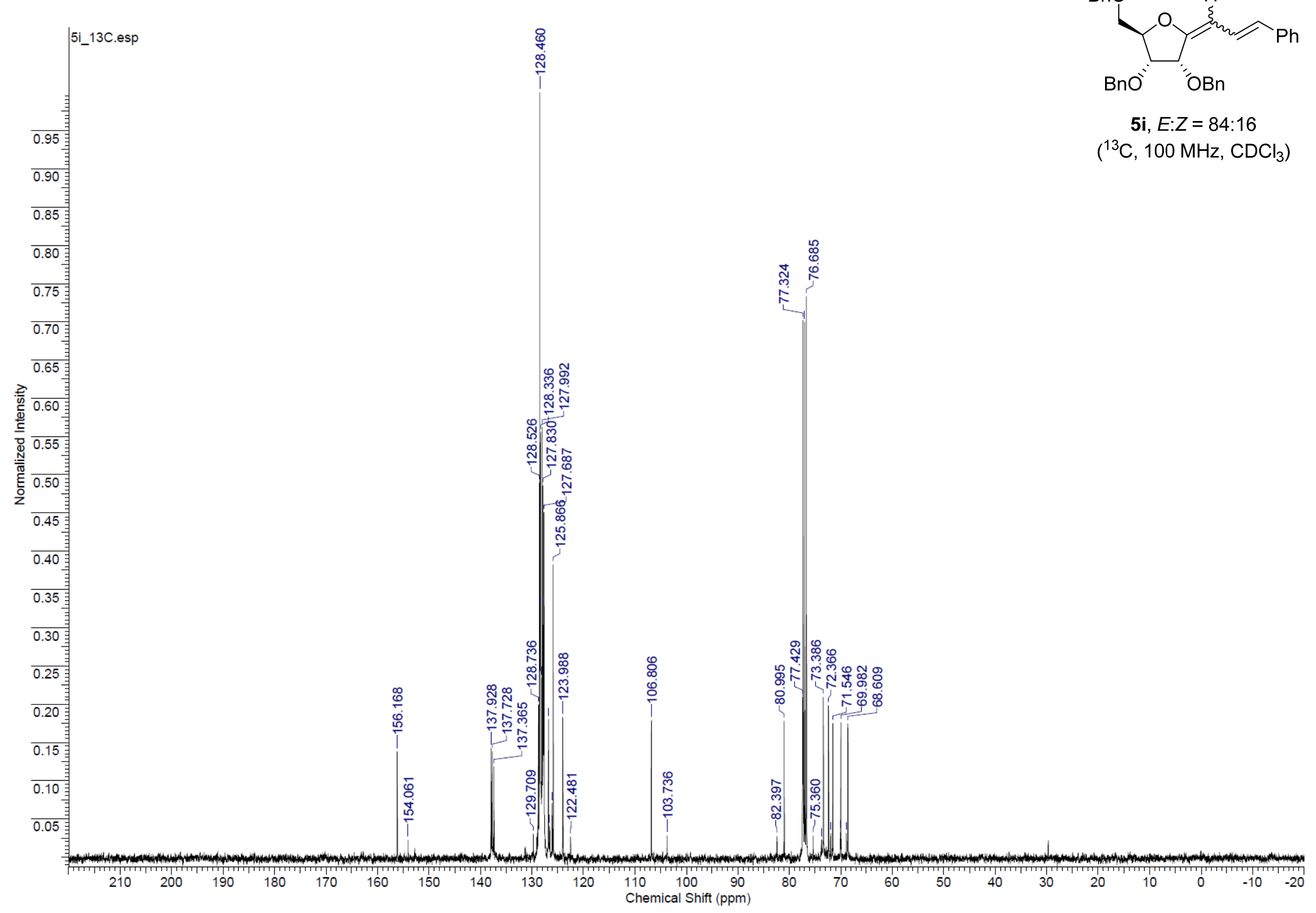




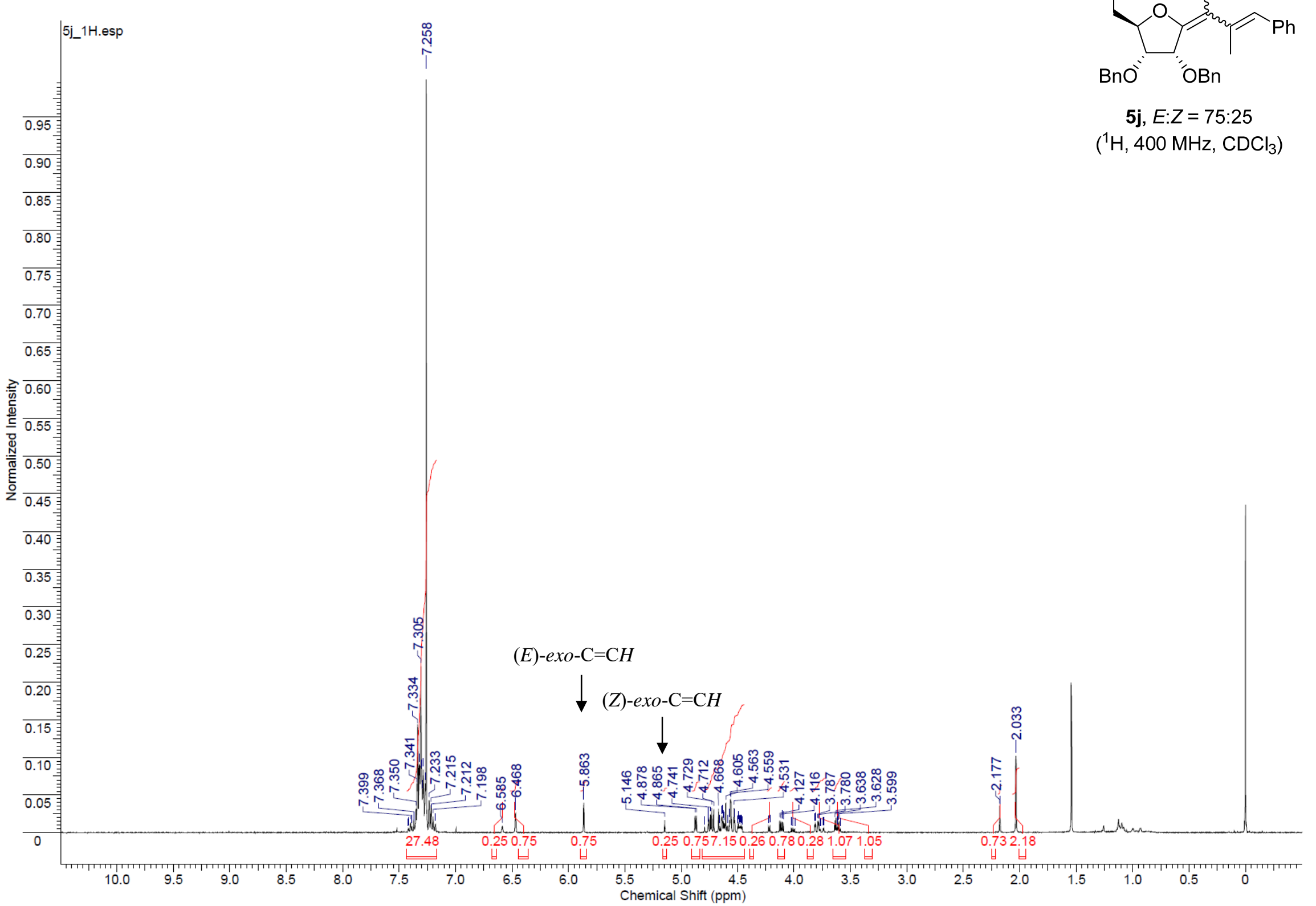




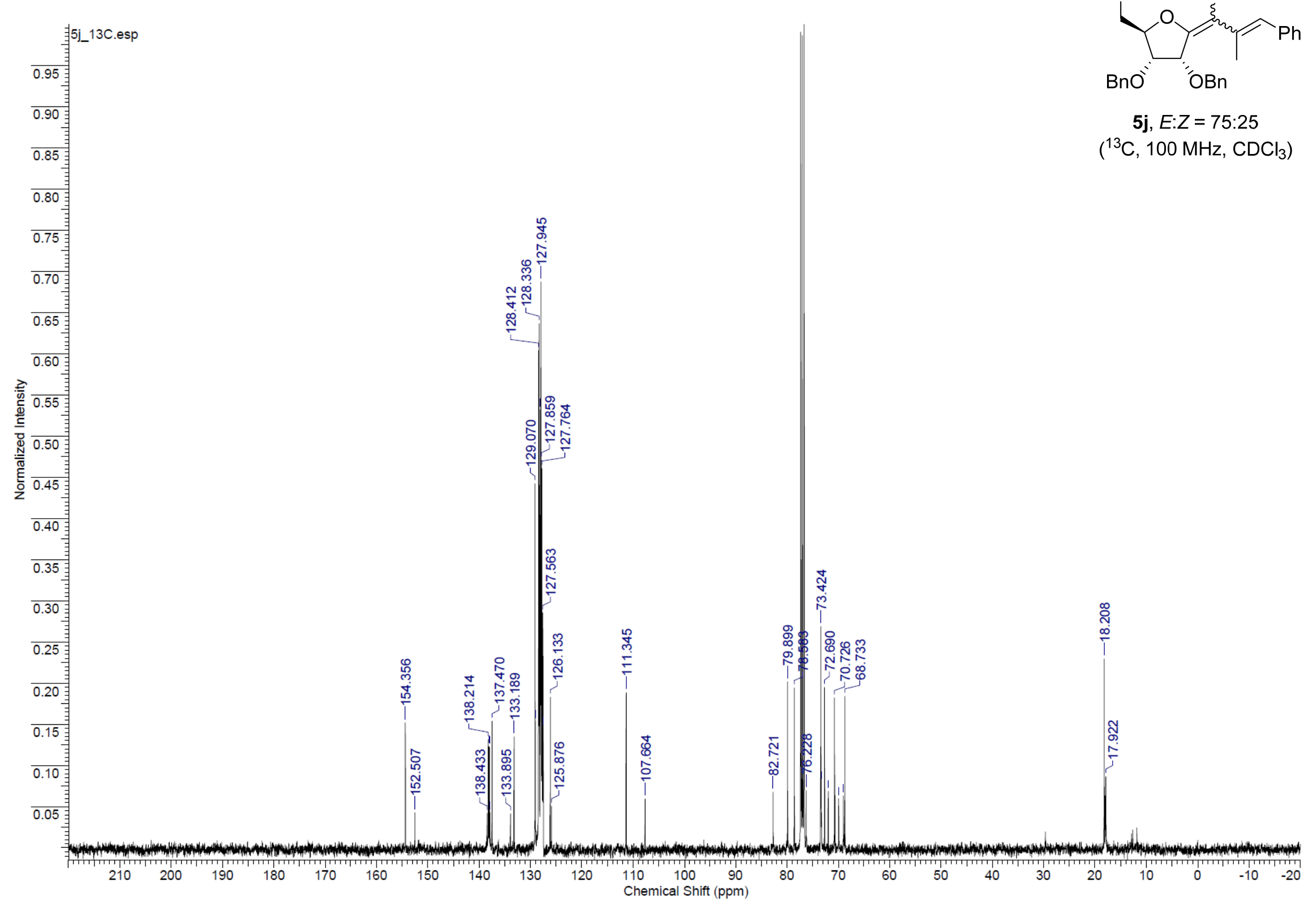




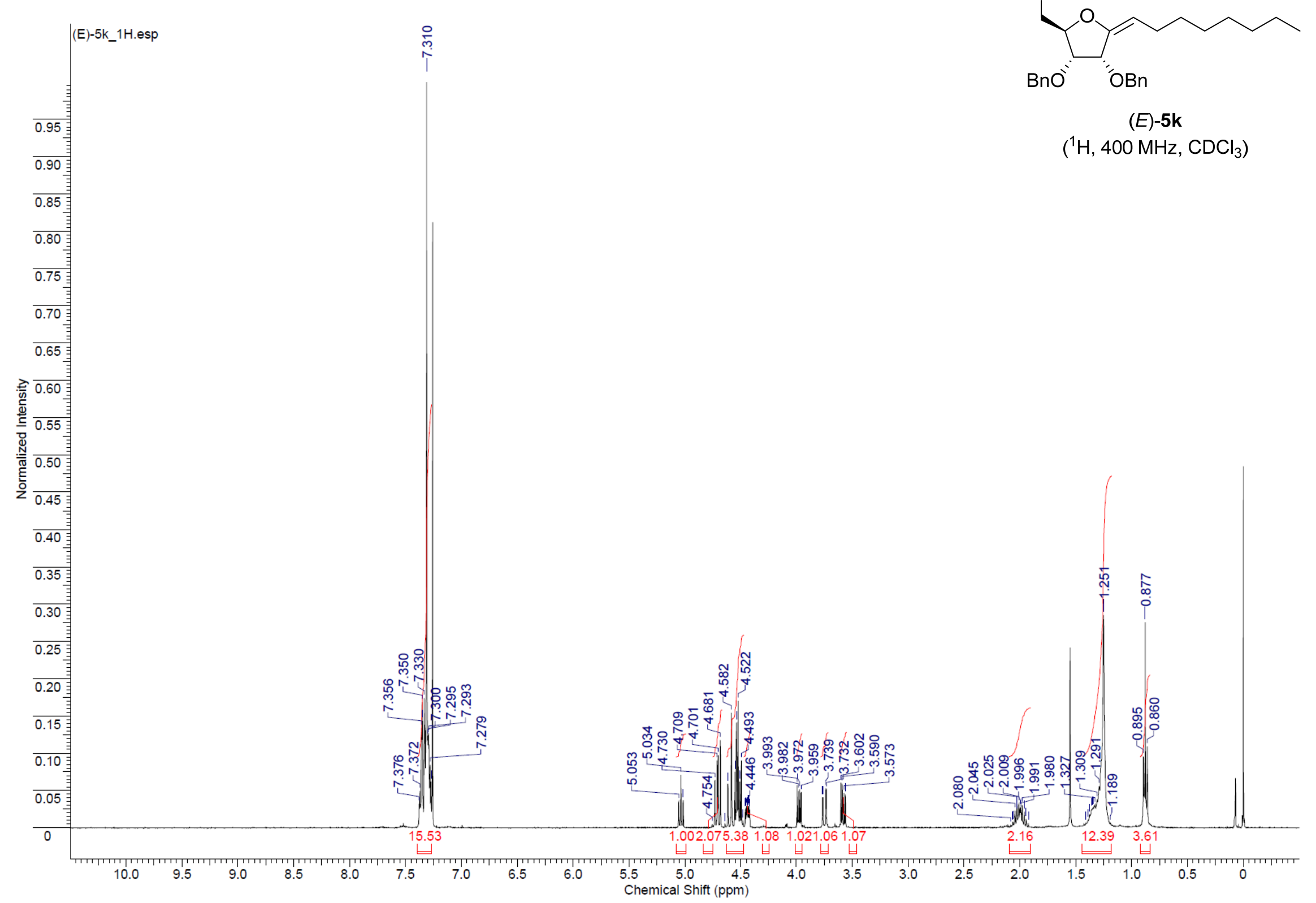




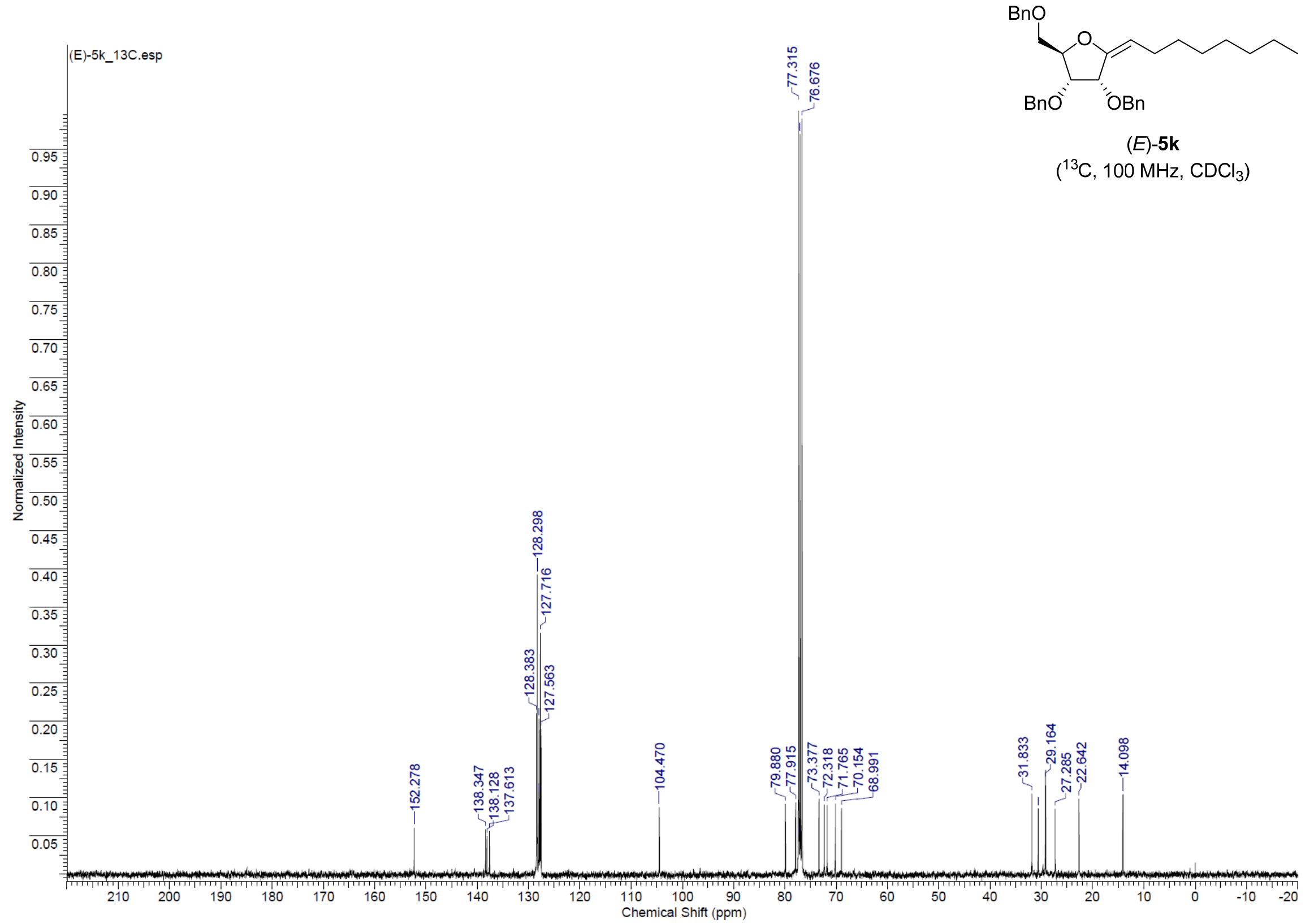




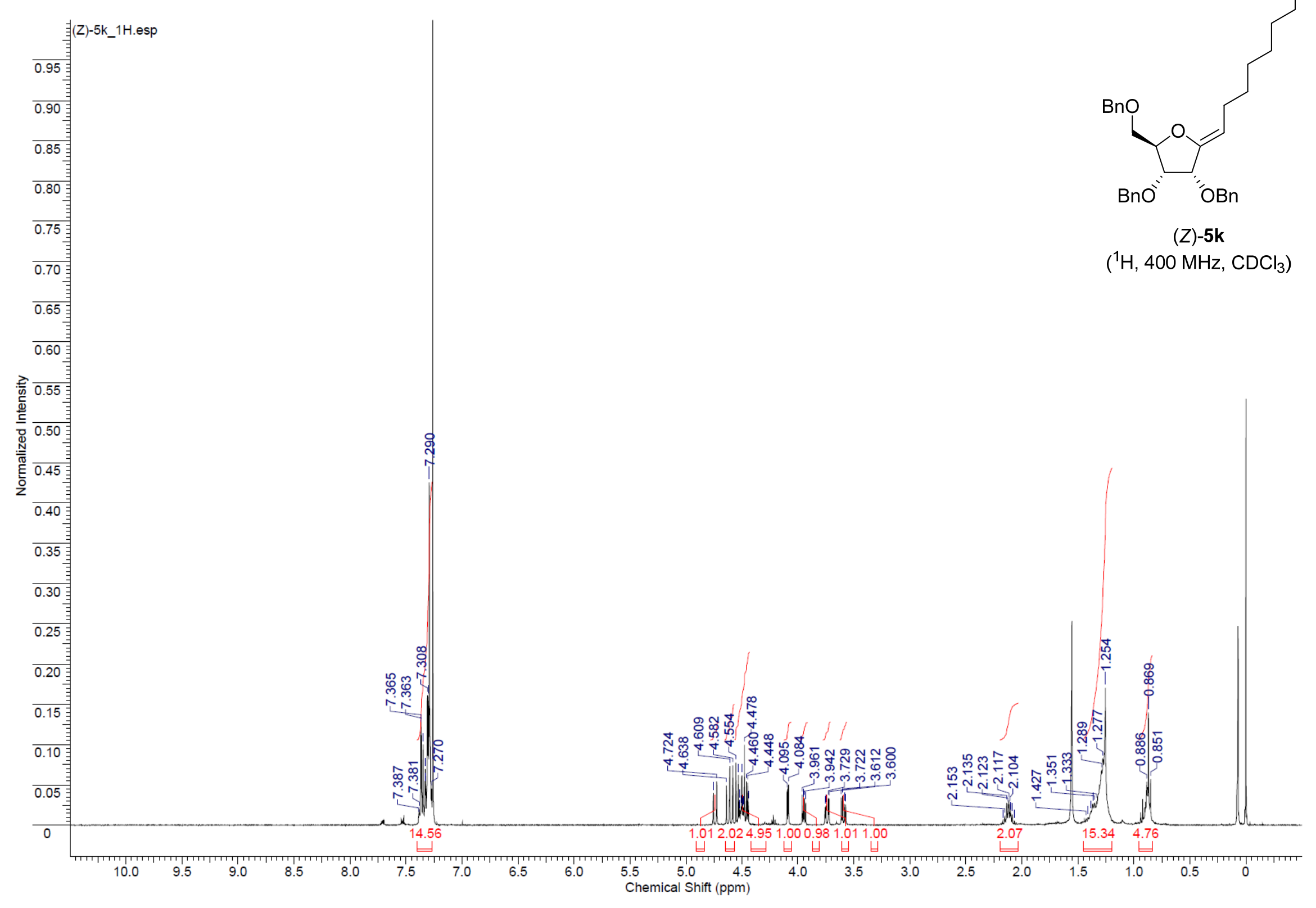




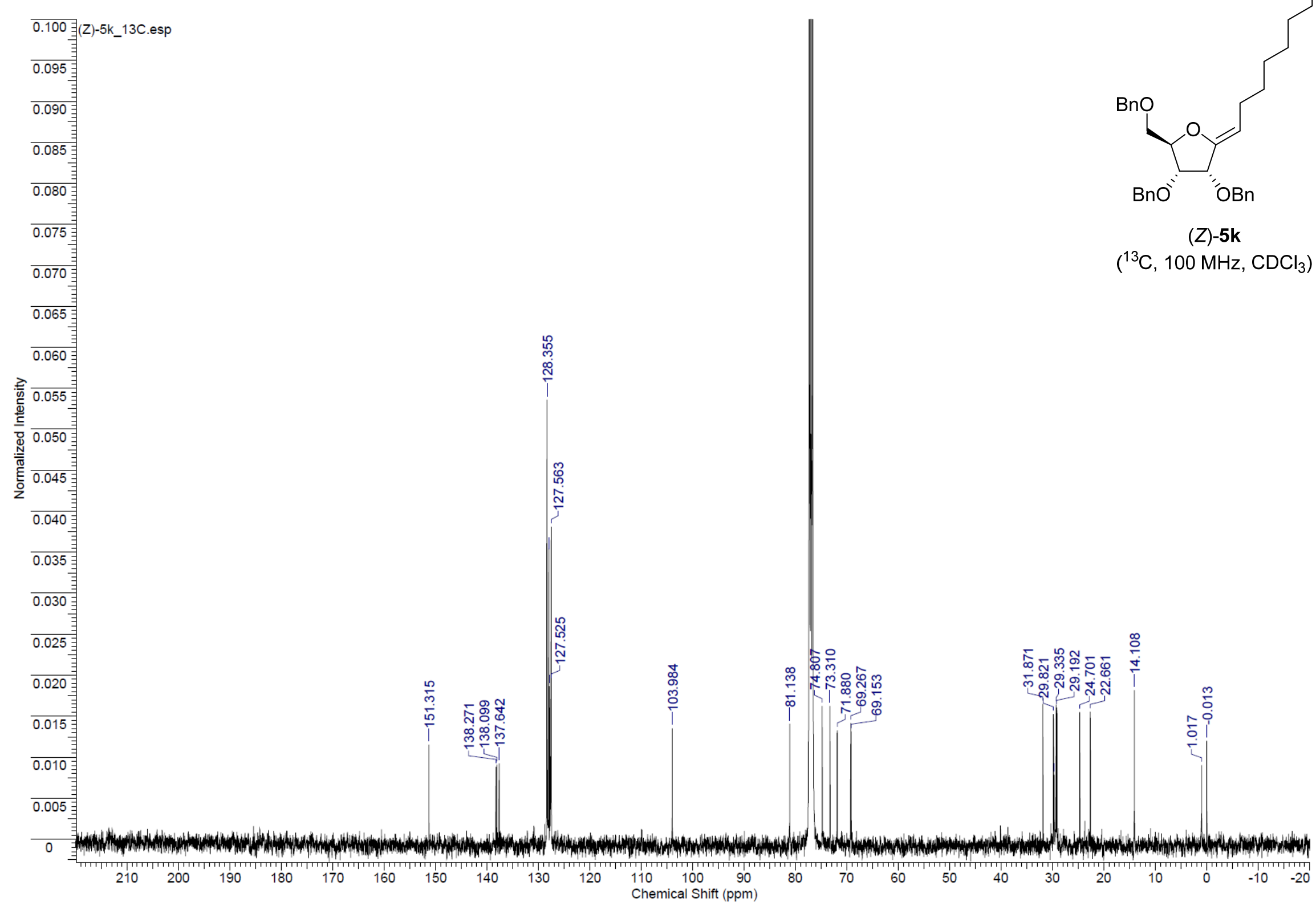




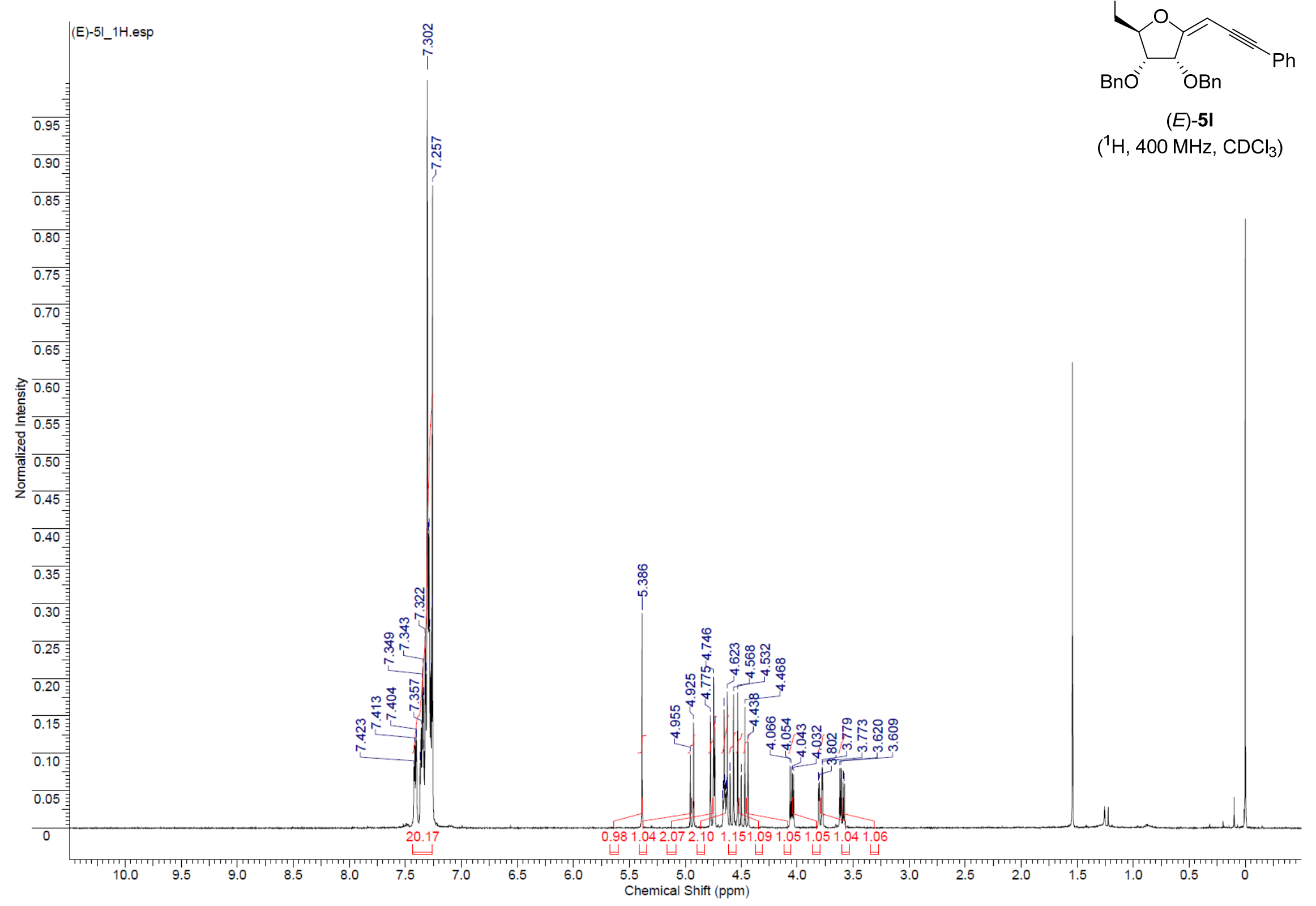




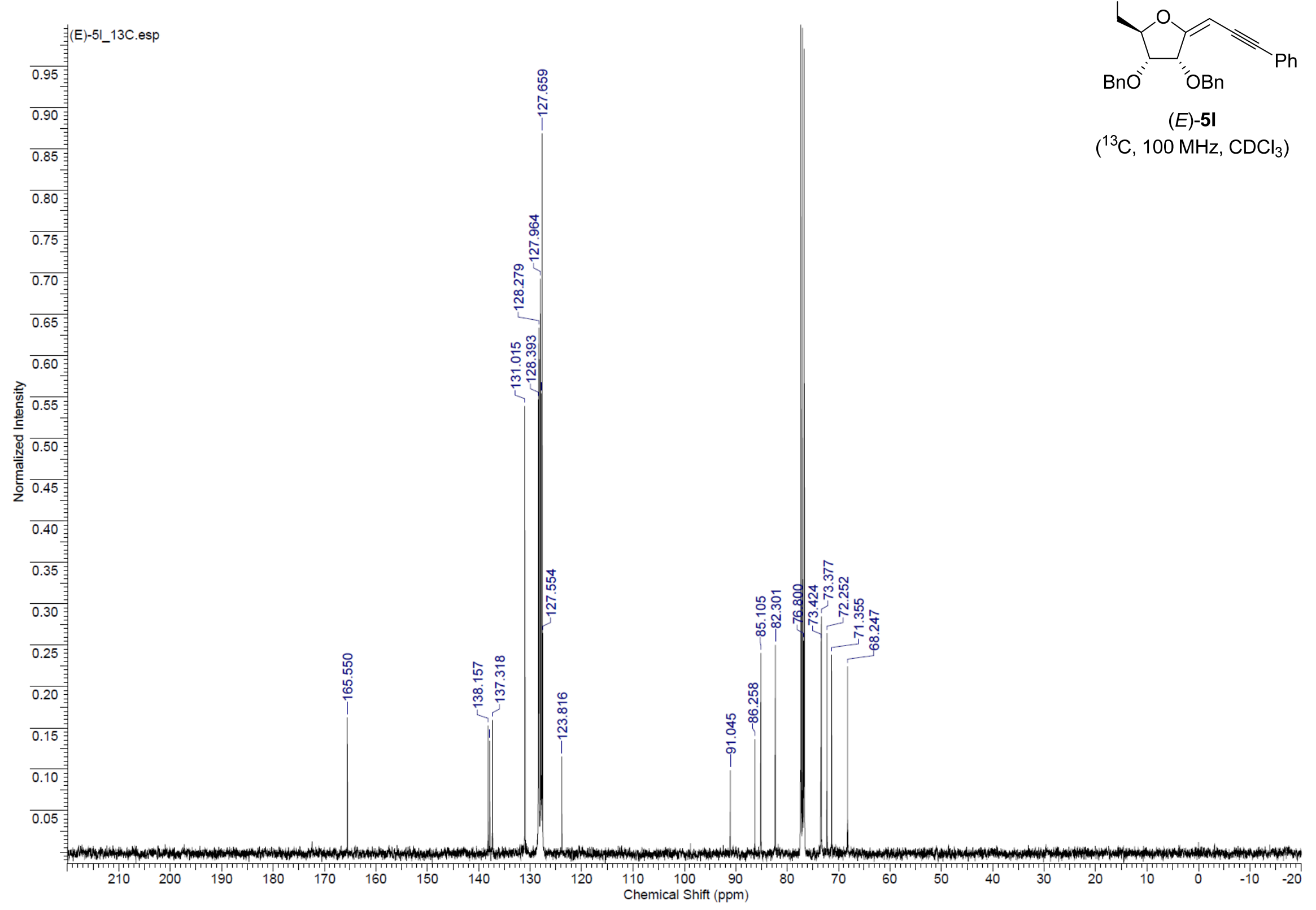




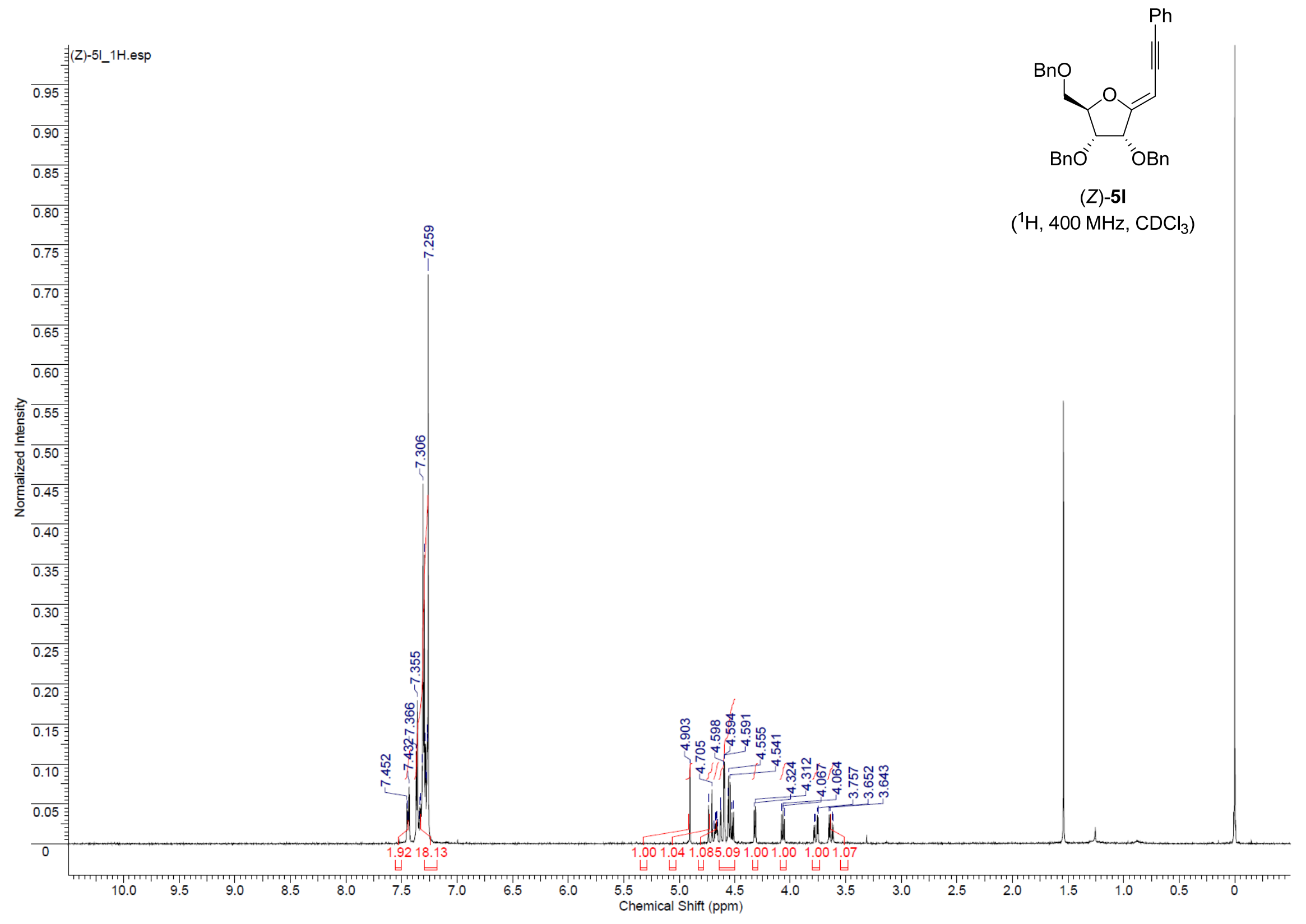




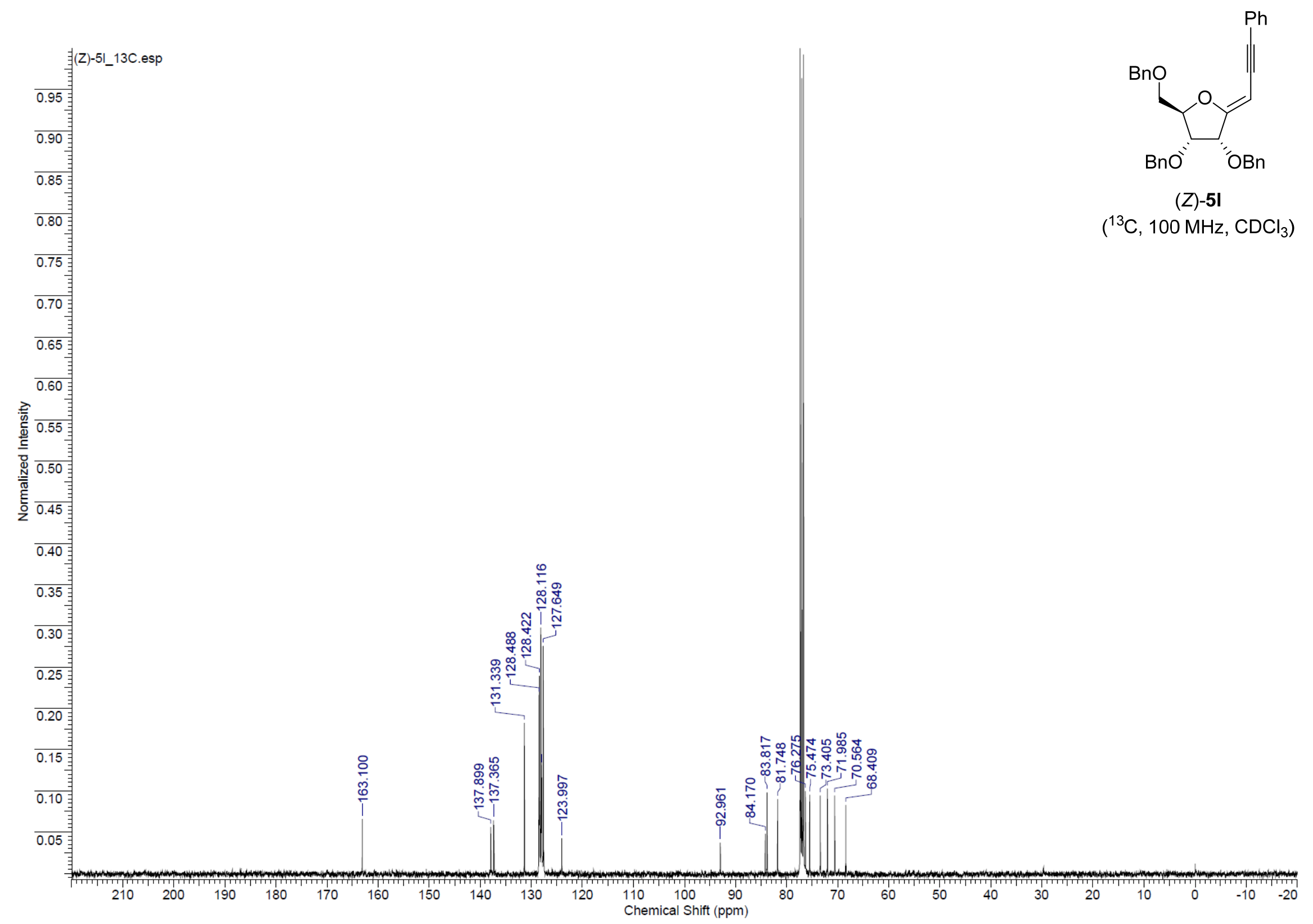




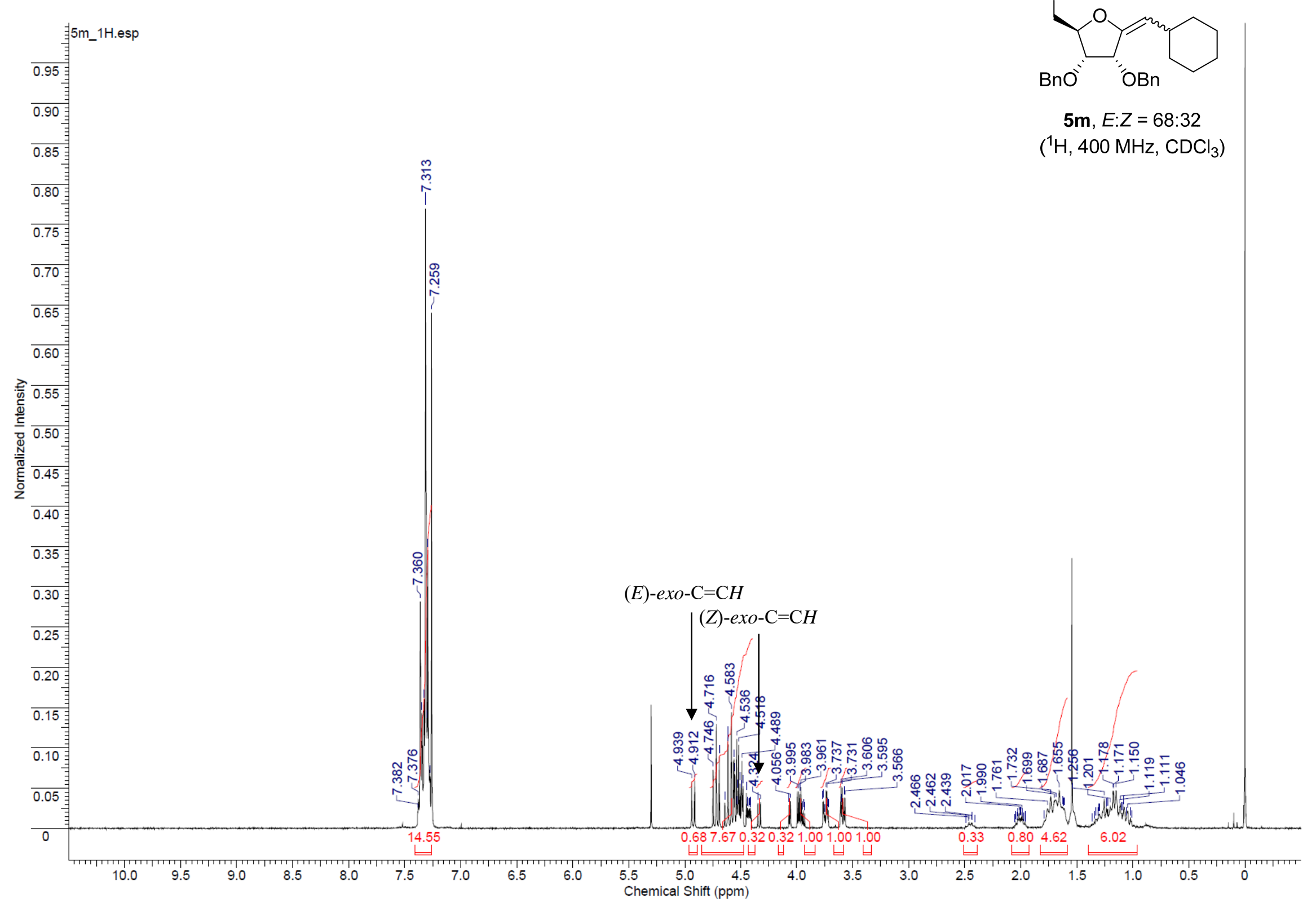




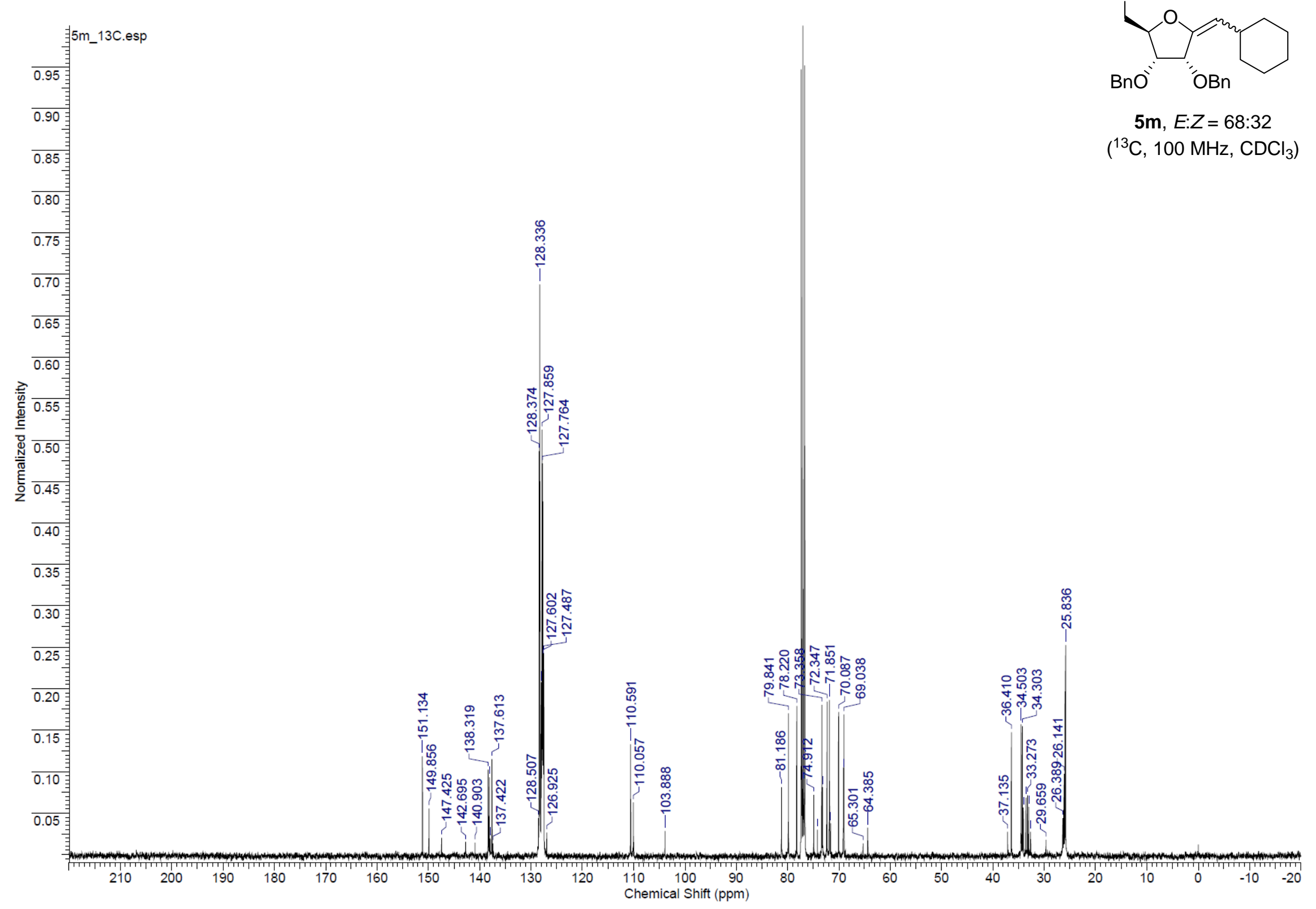




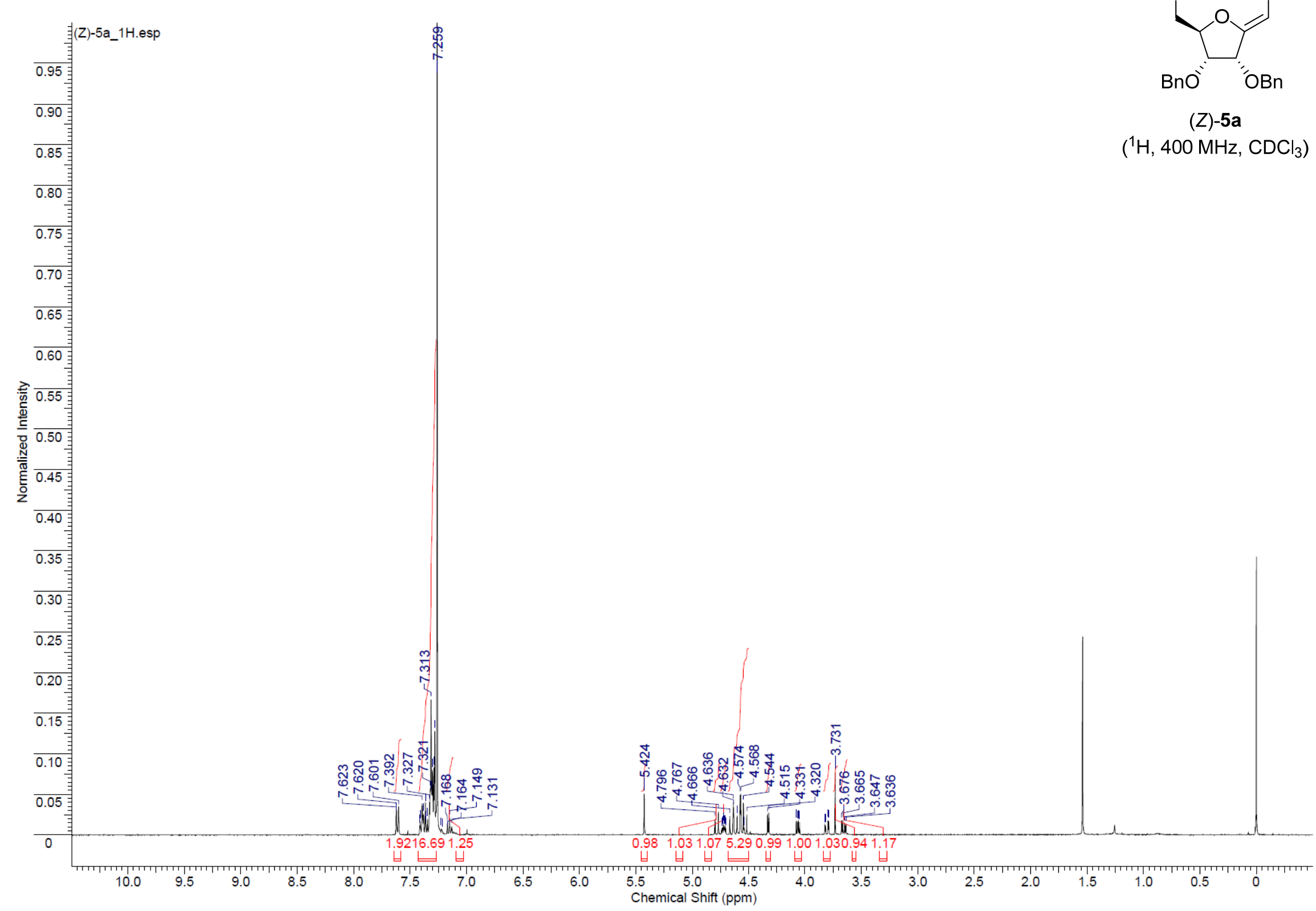




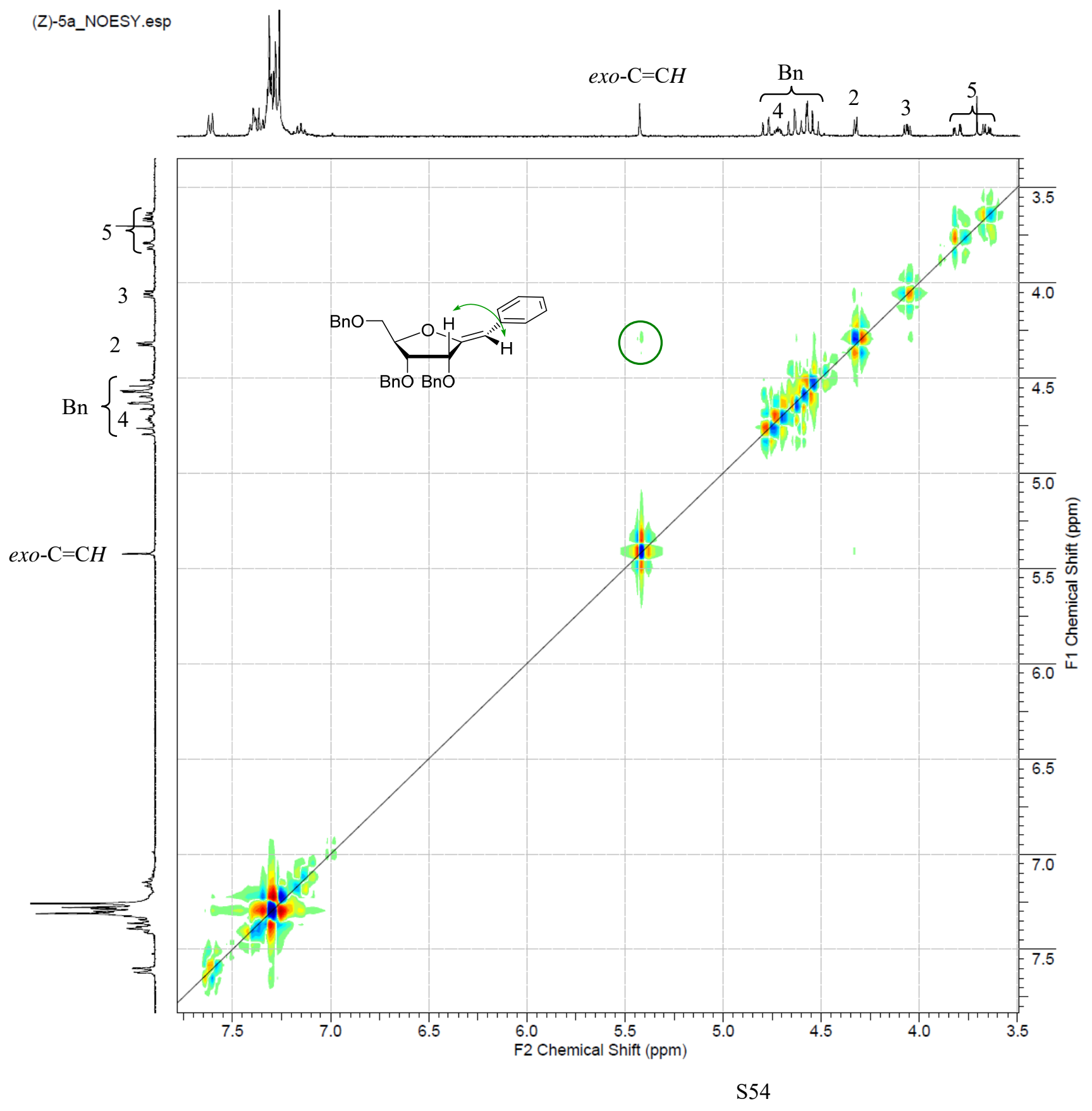

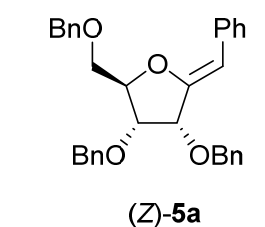

(NOESY, $400 \mathrm{MHz}, \mathrm{CDCl}_{3}$ ) 


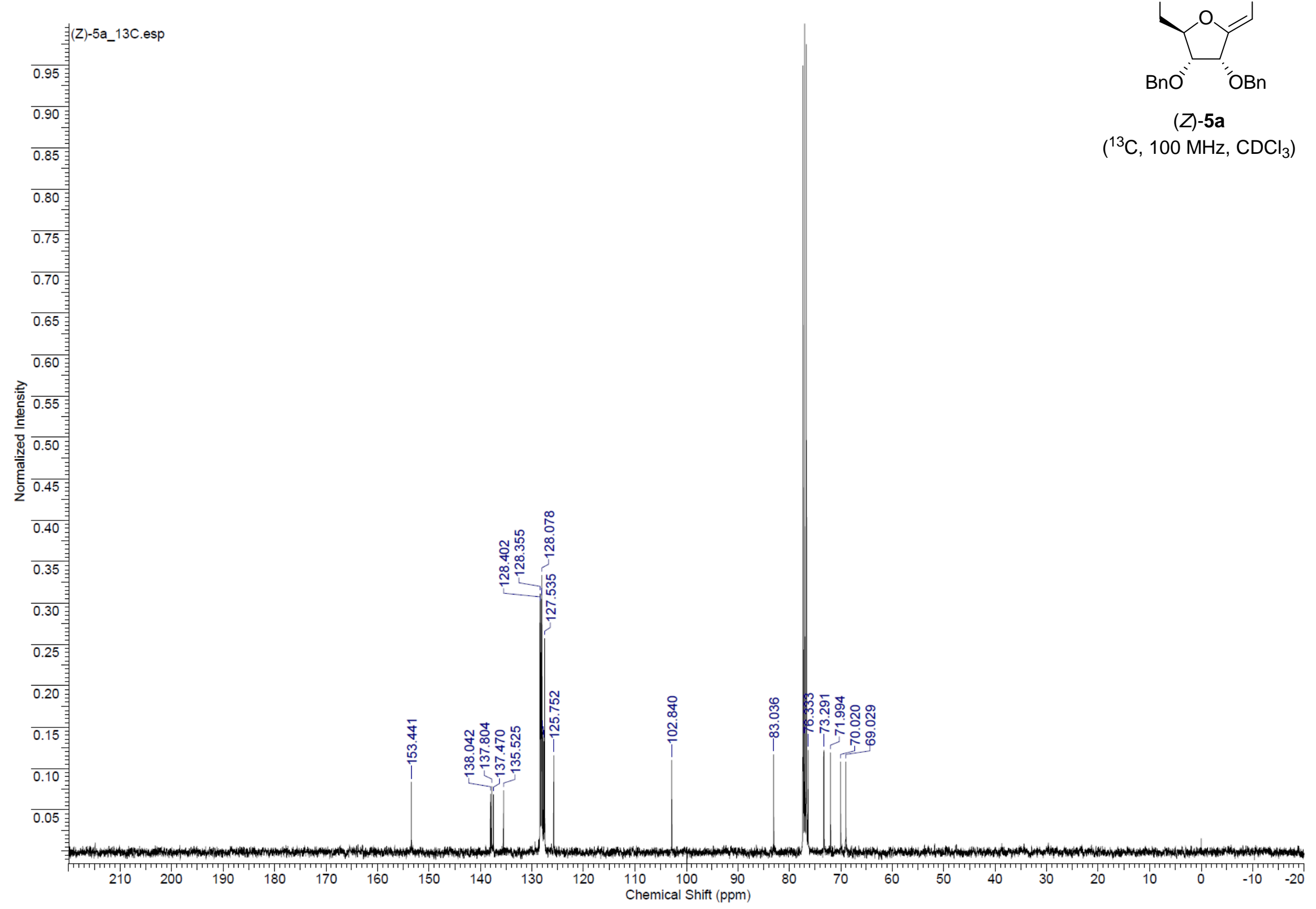




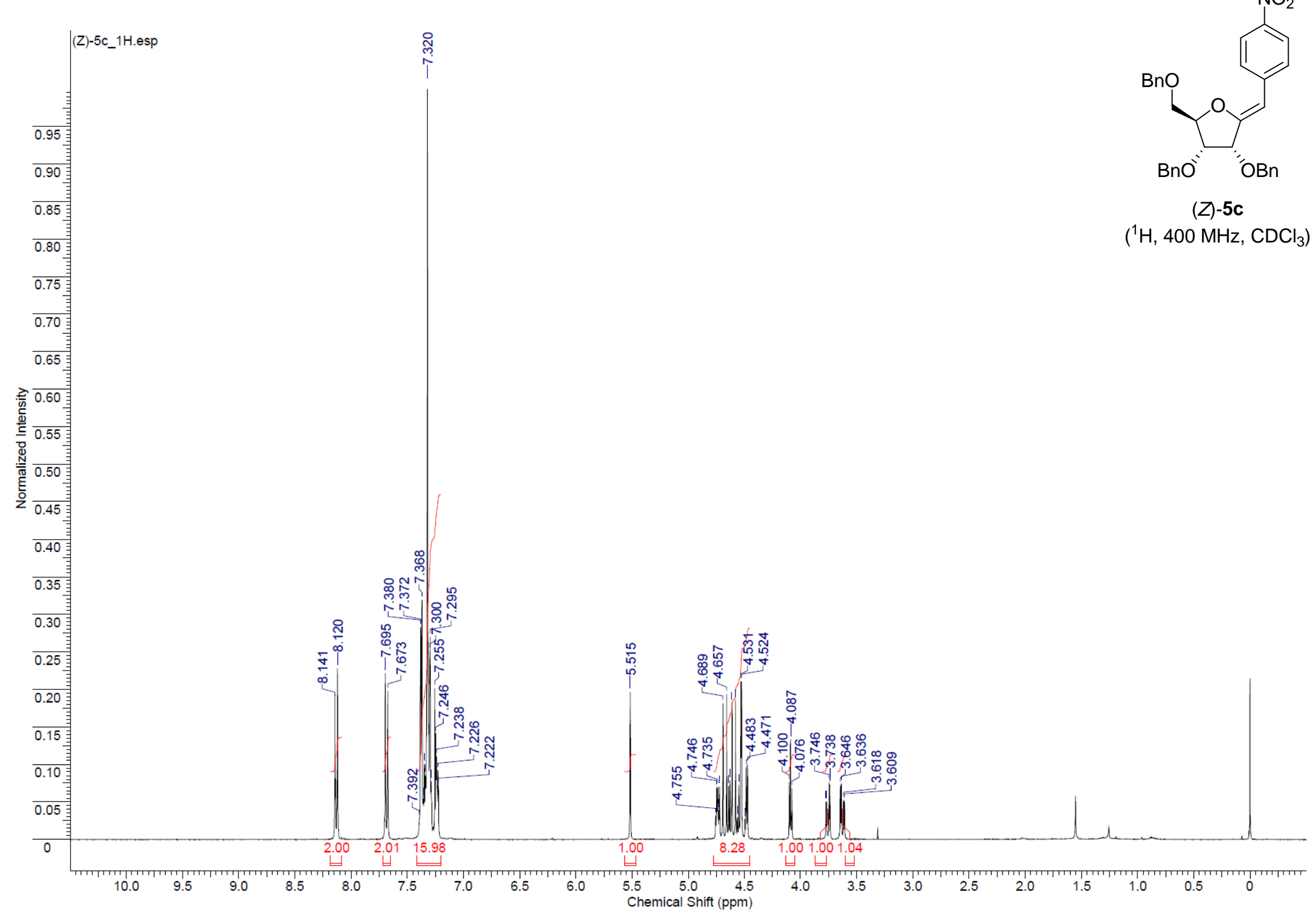




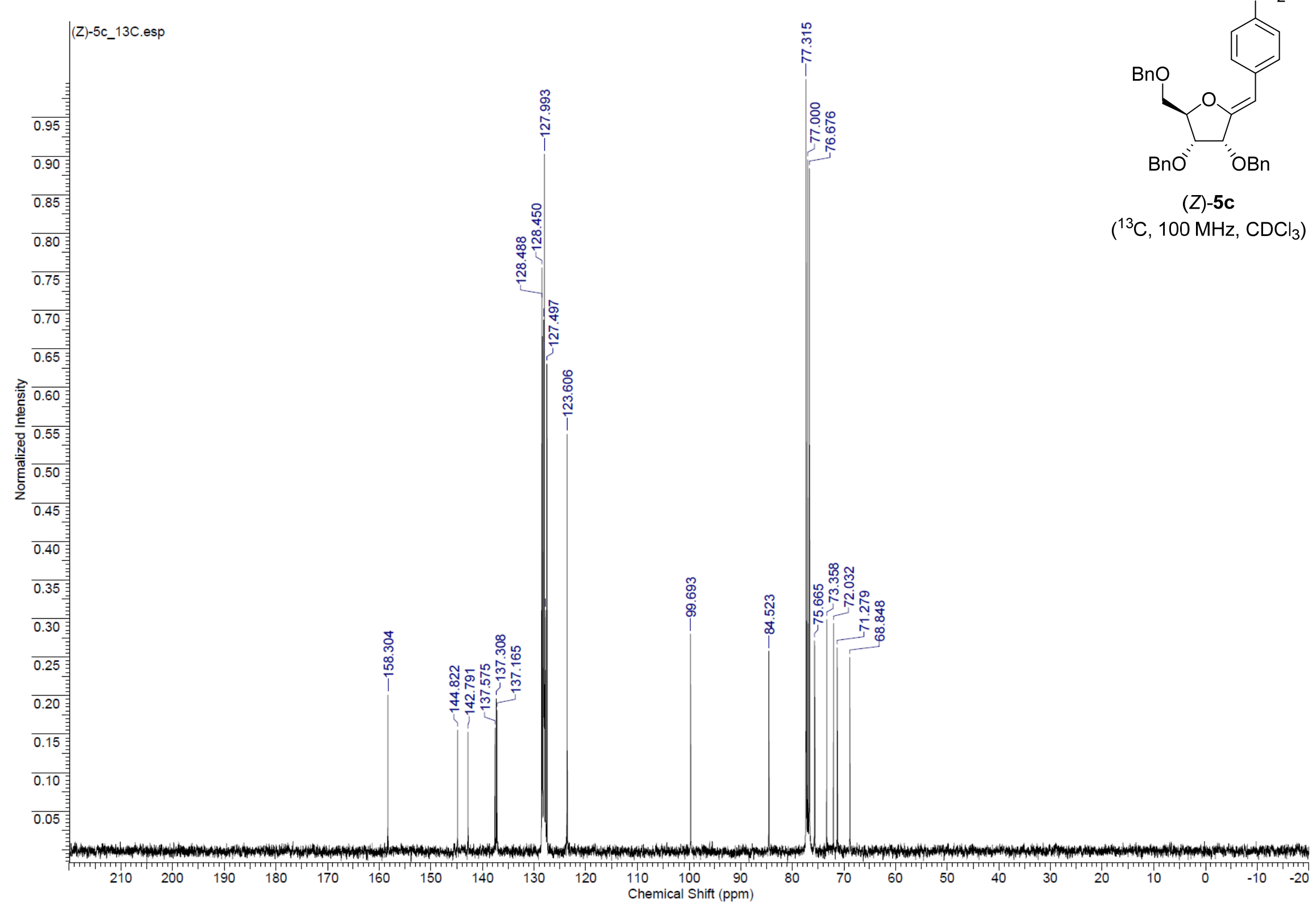




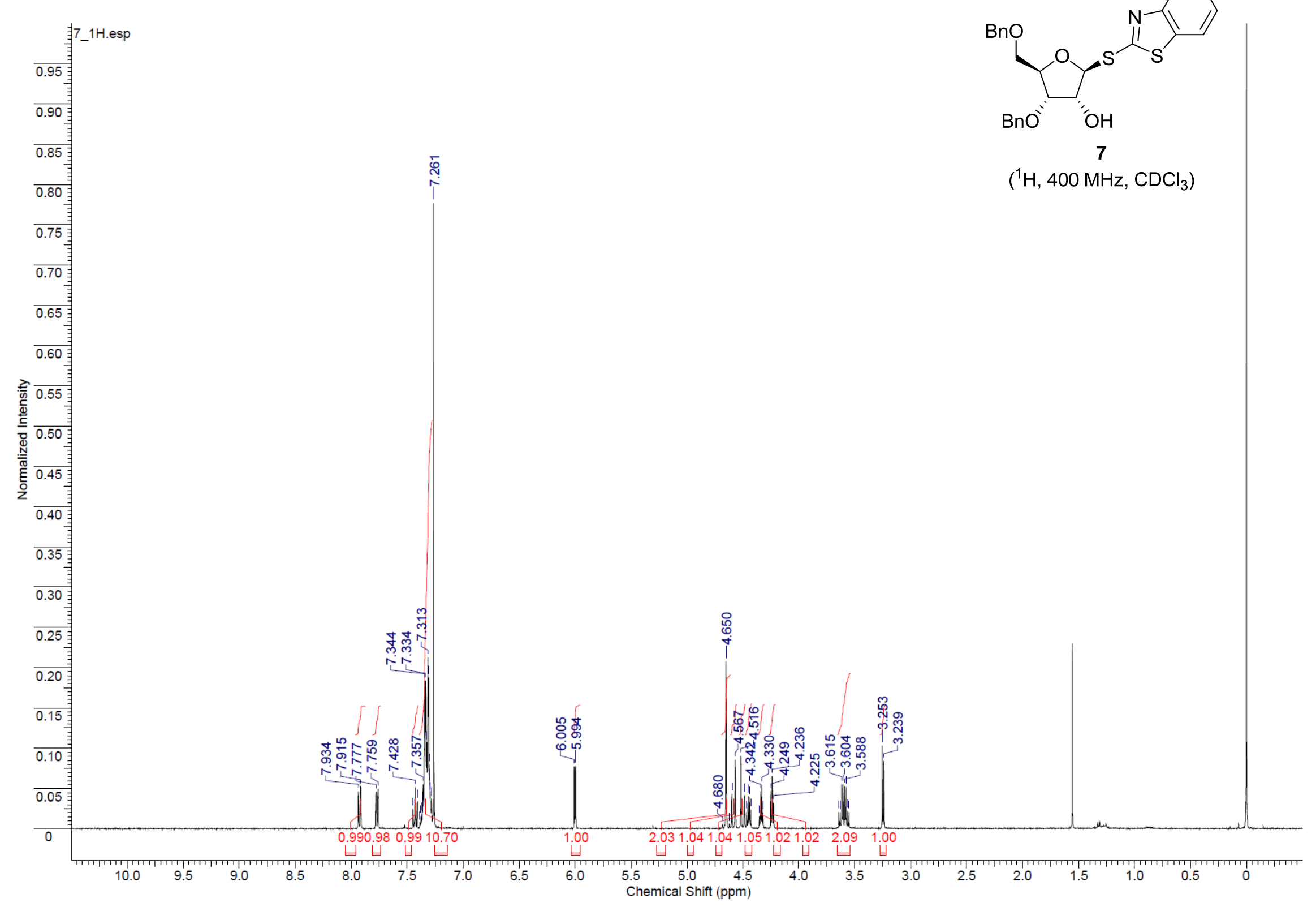




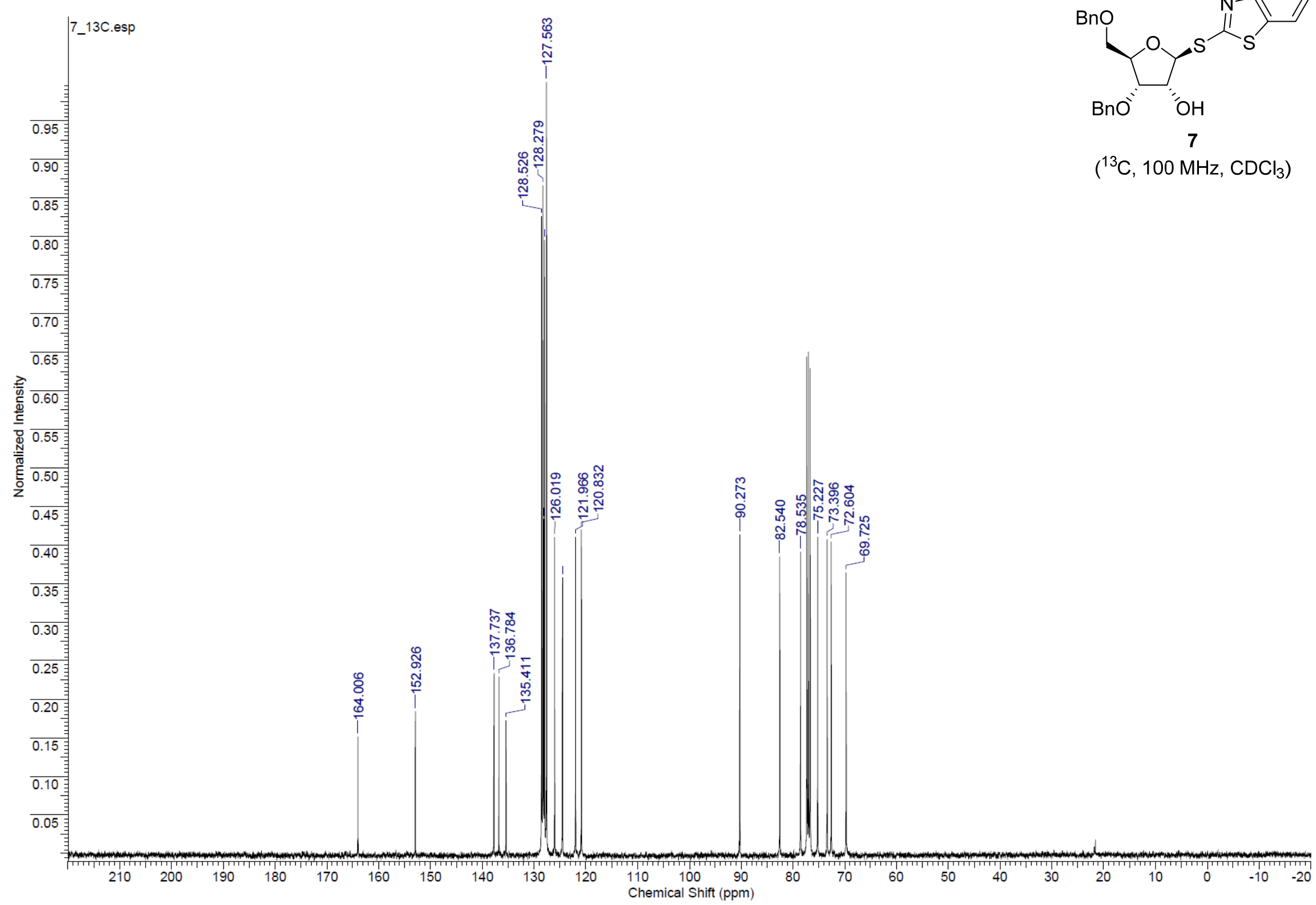




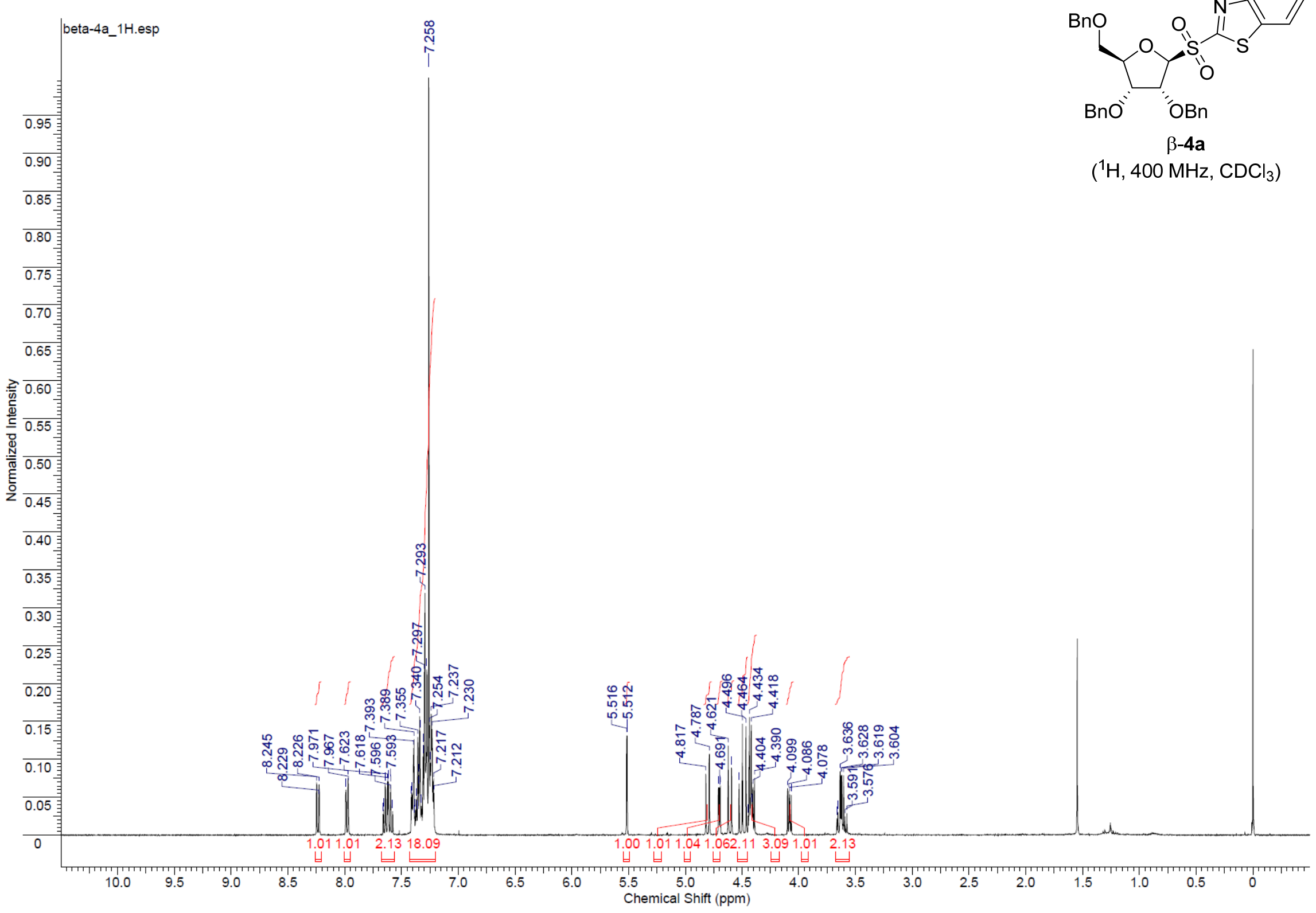




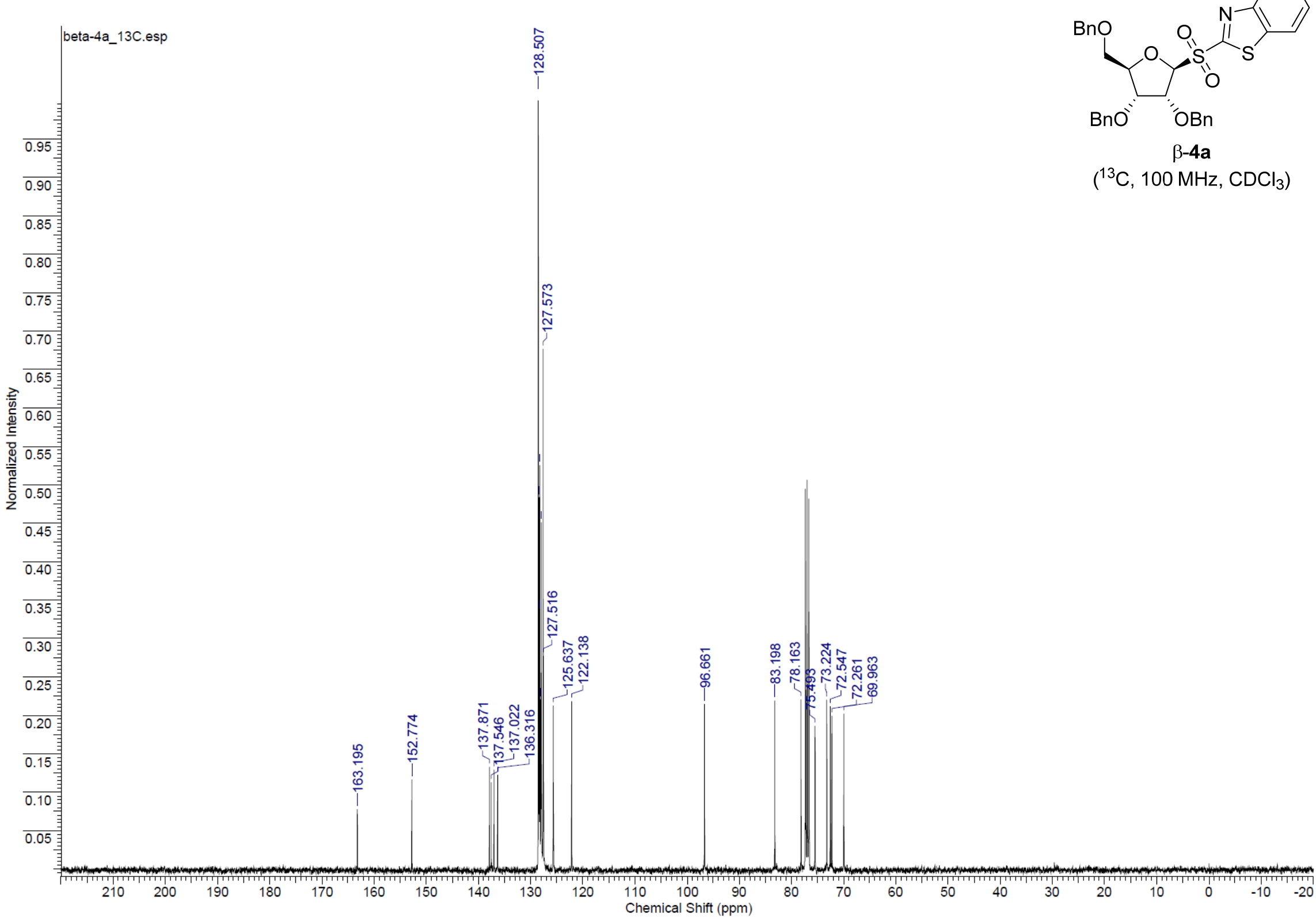




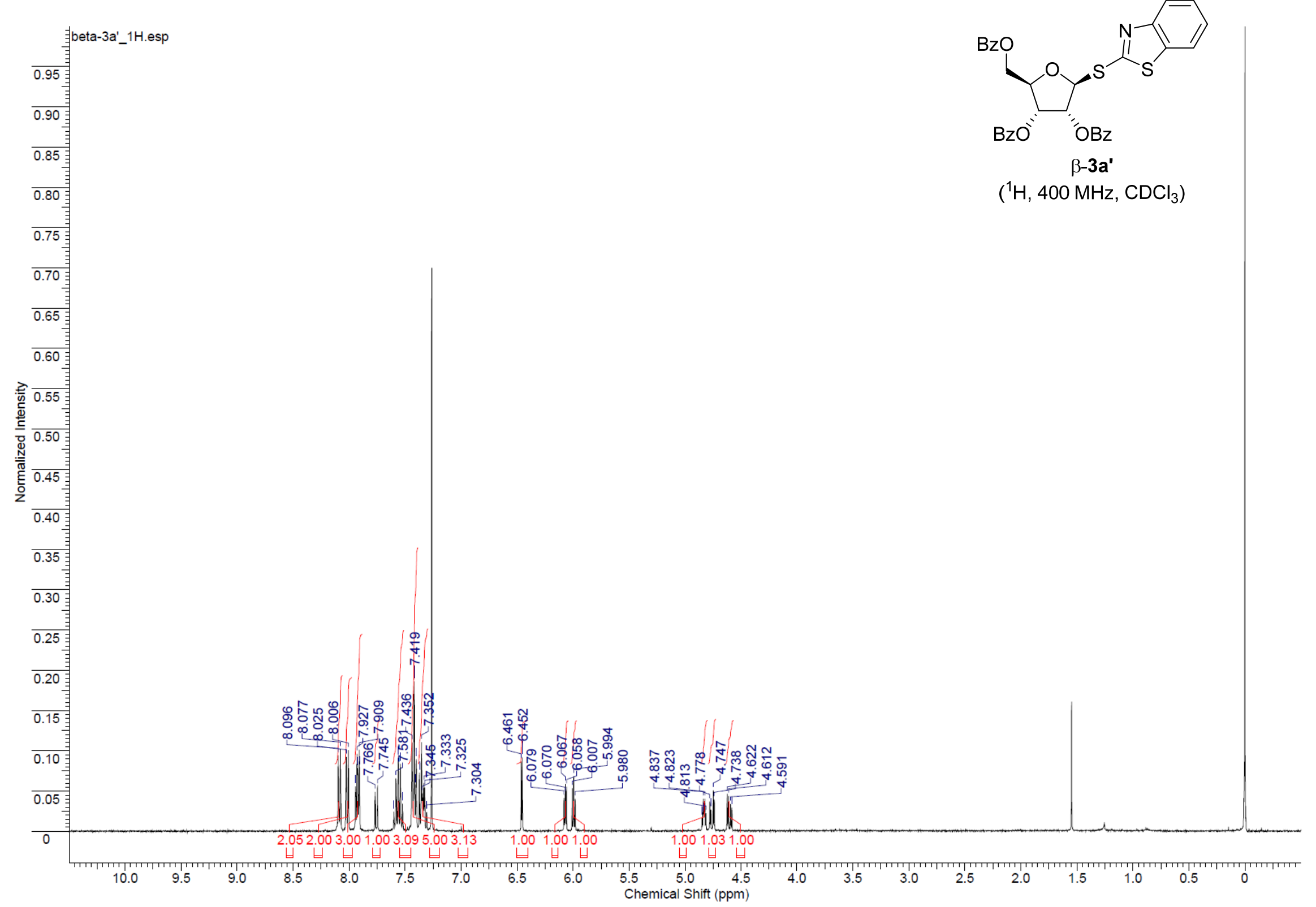




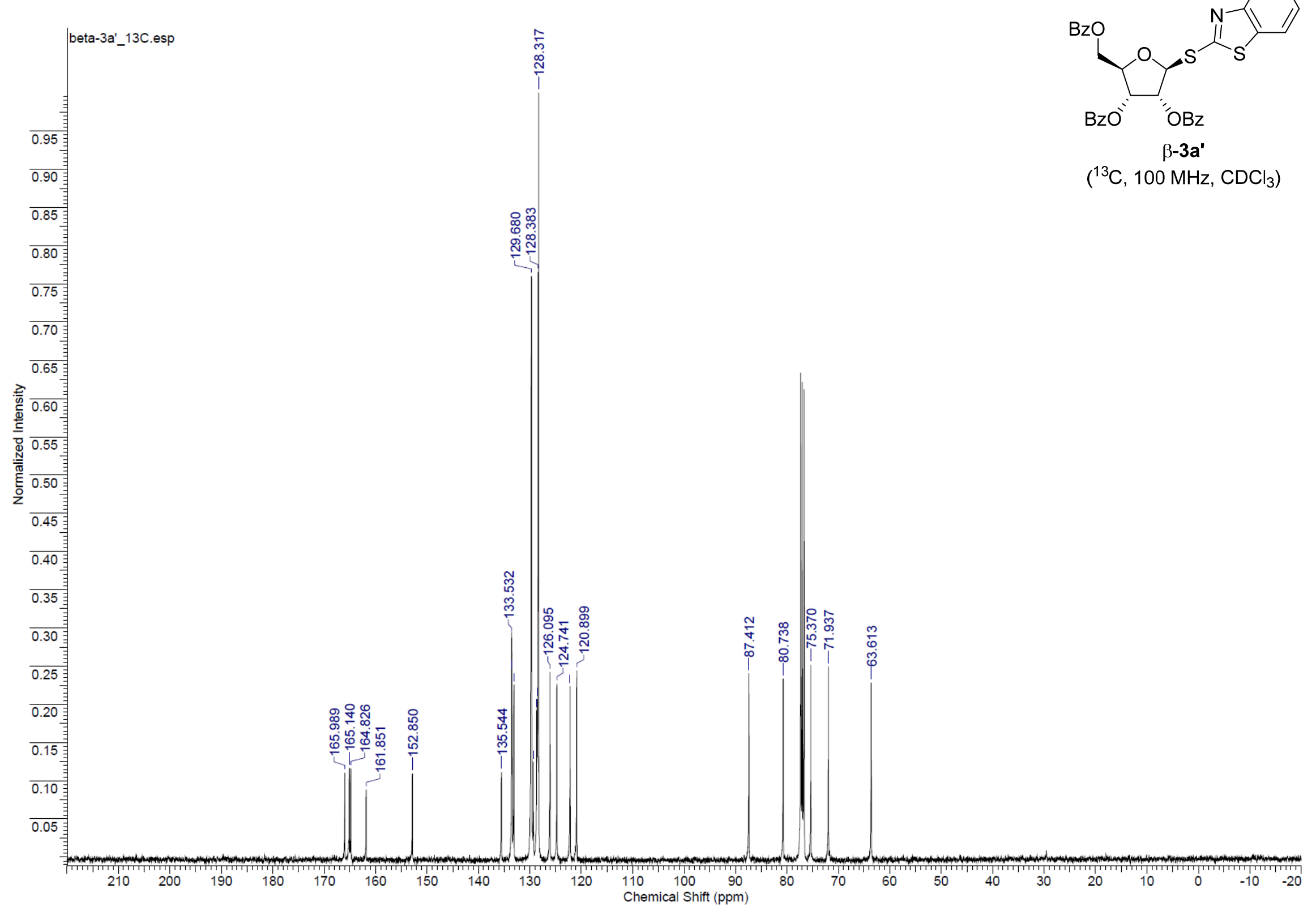




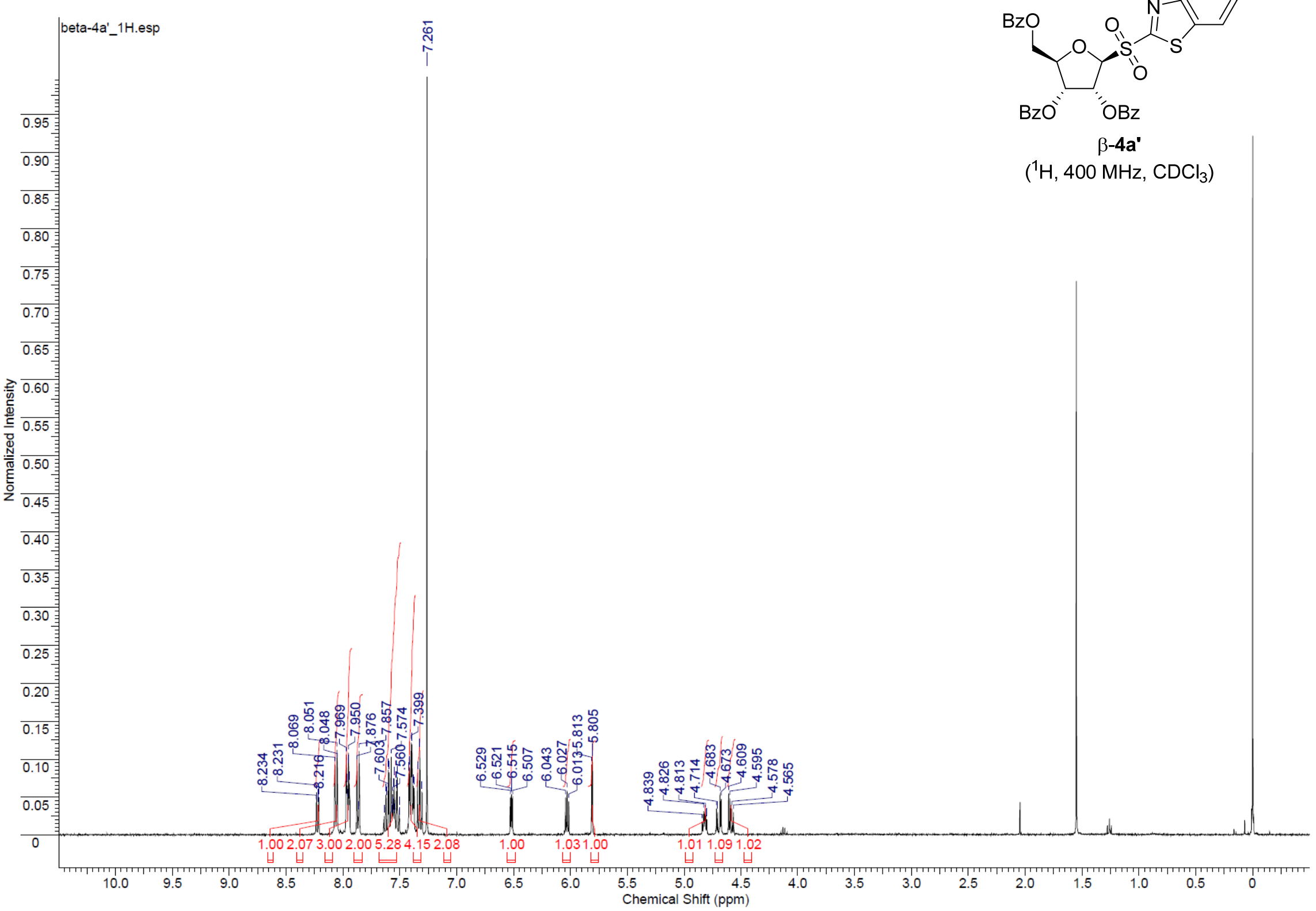




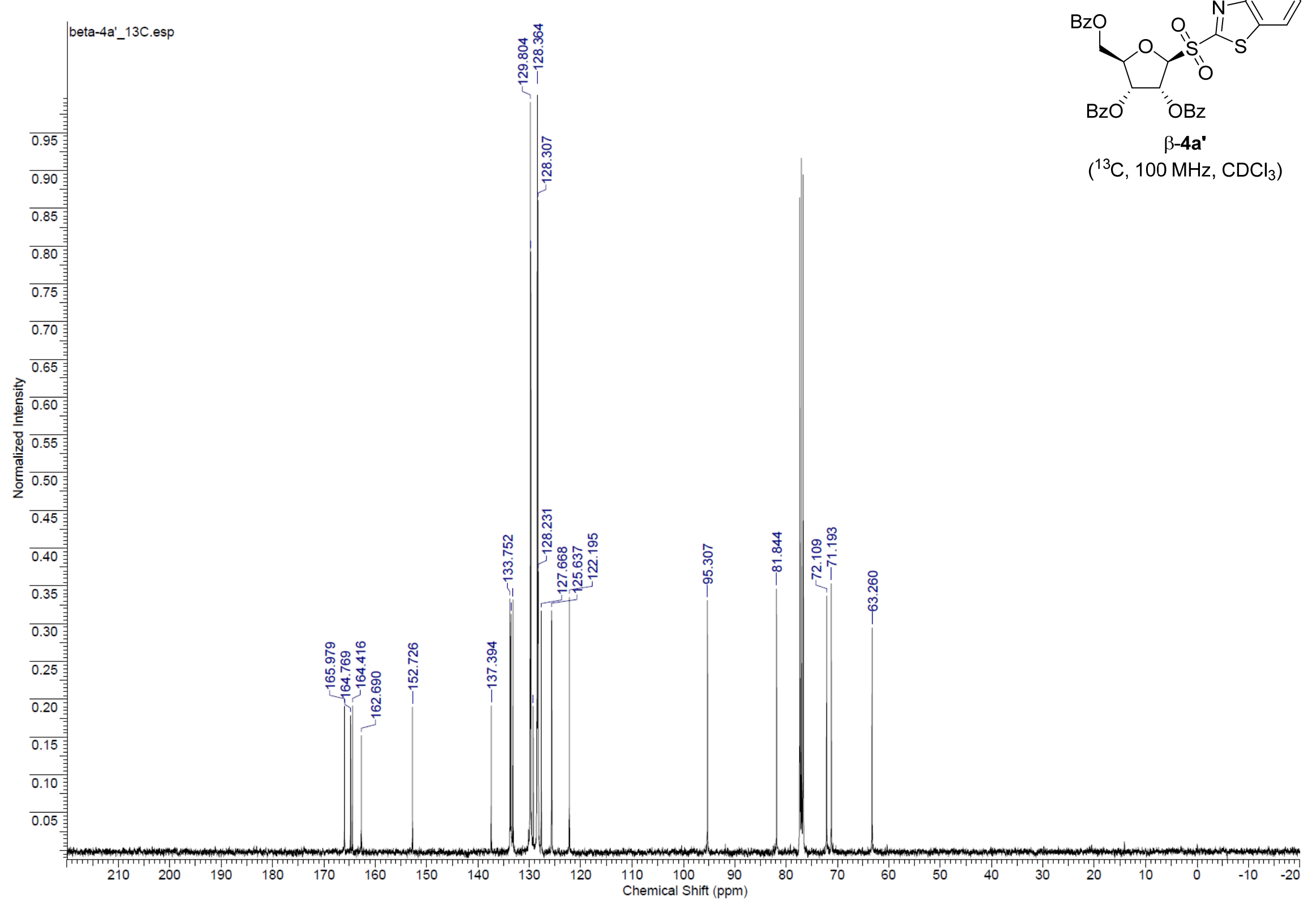




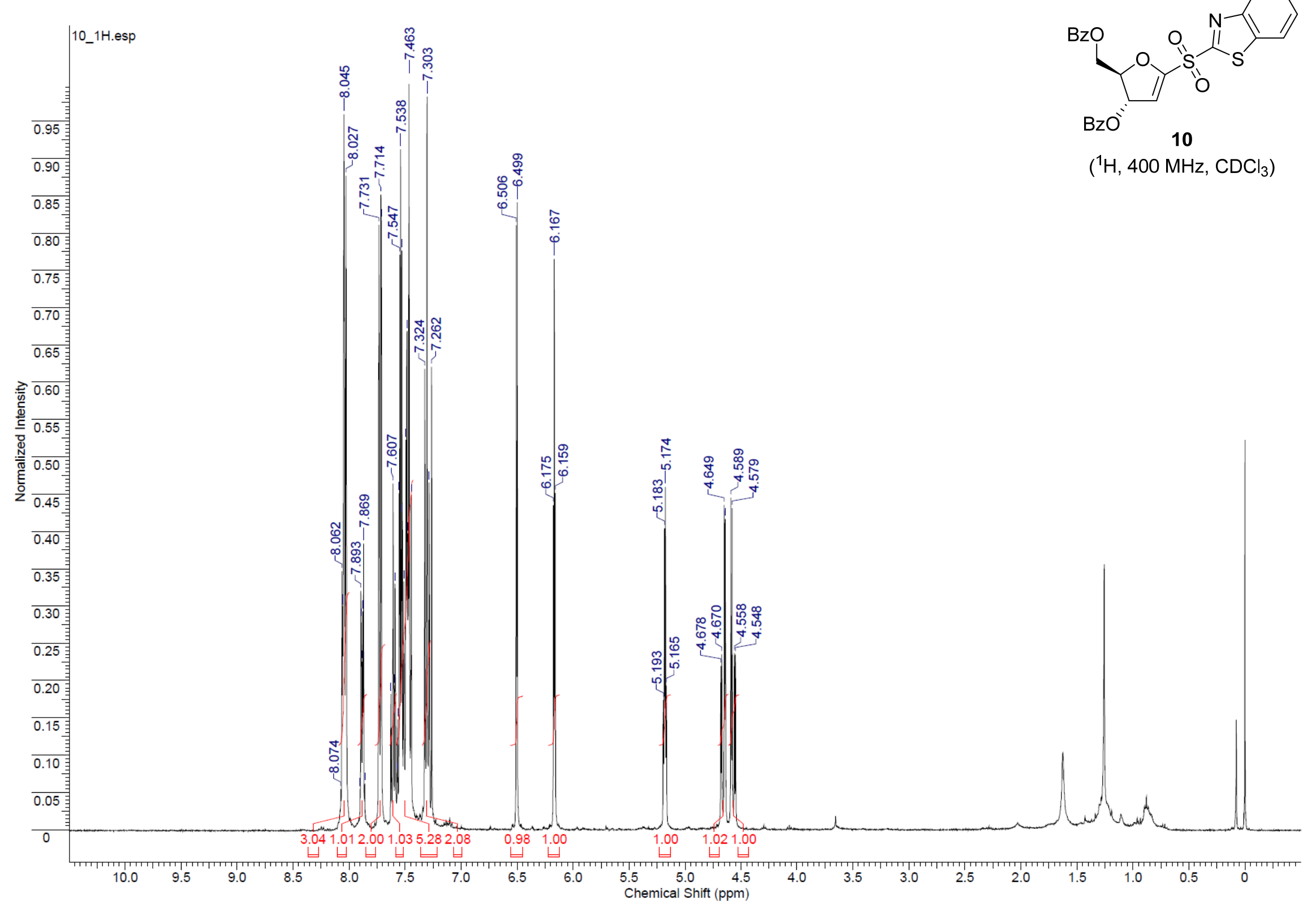




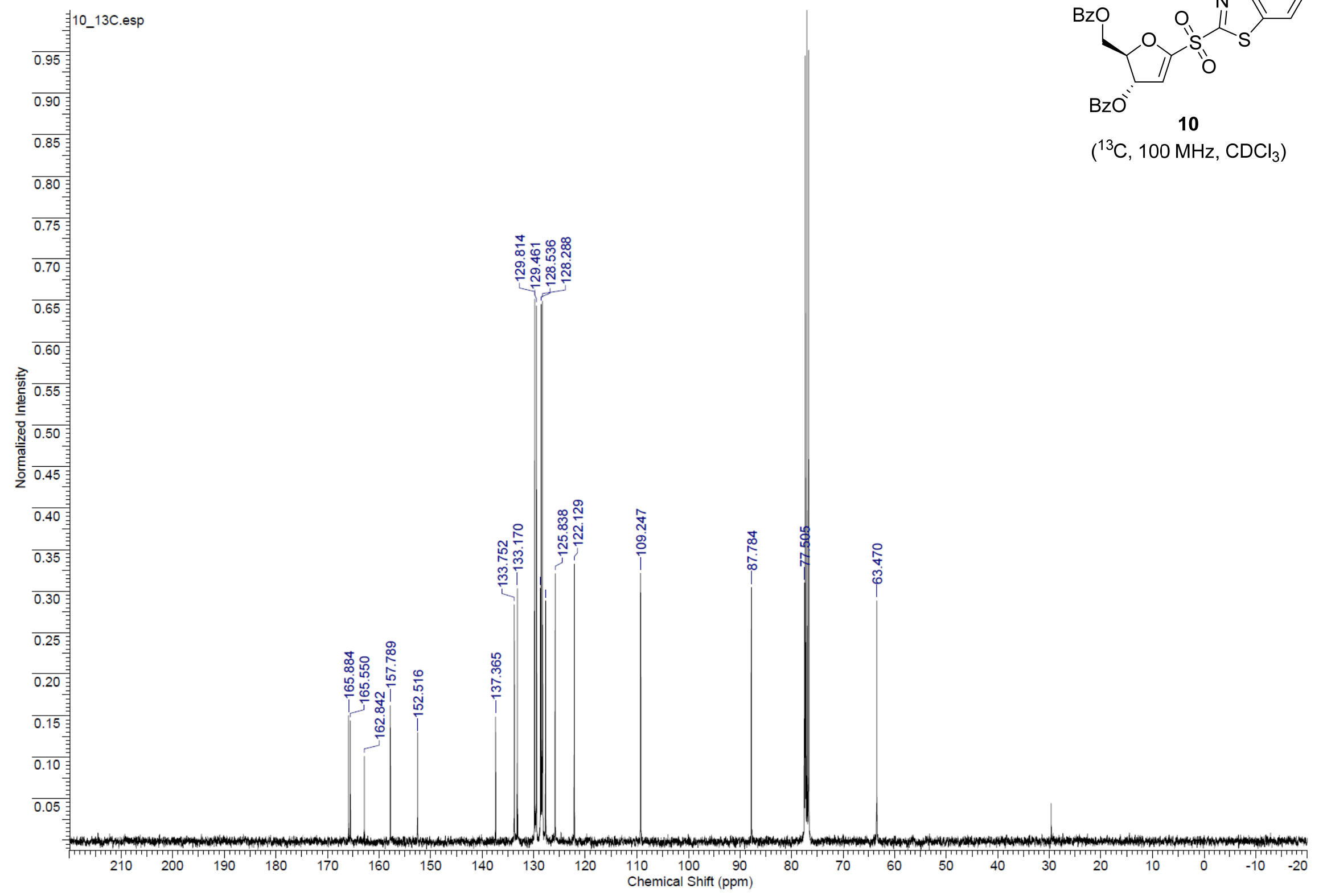




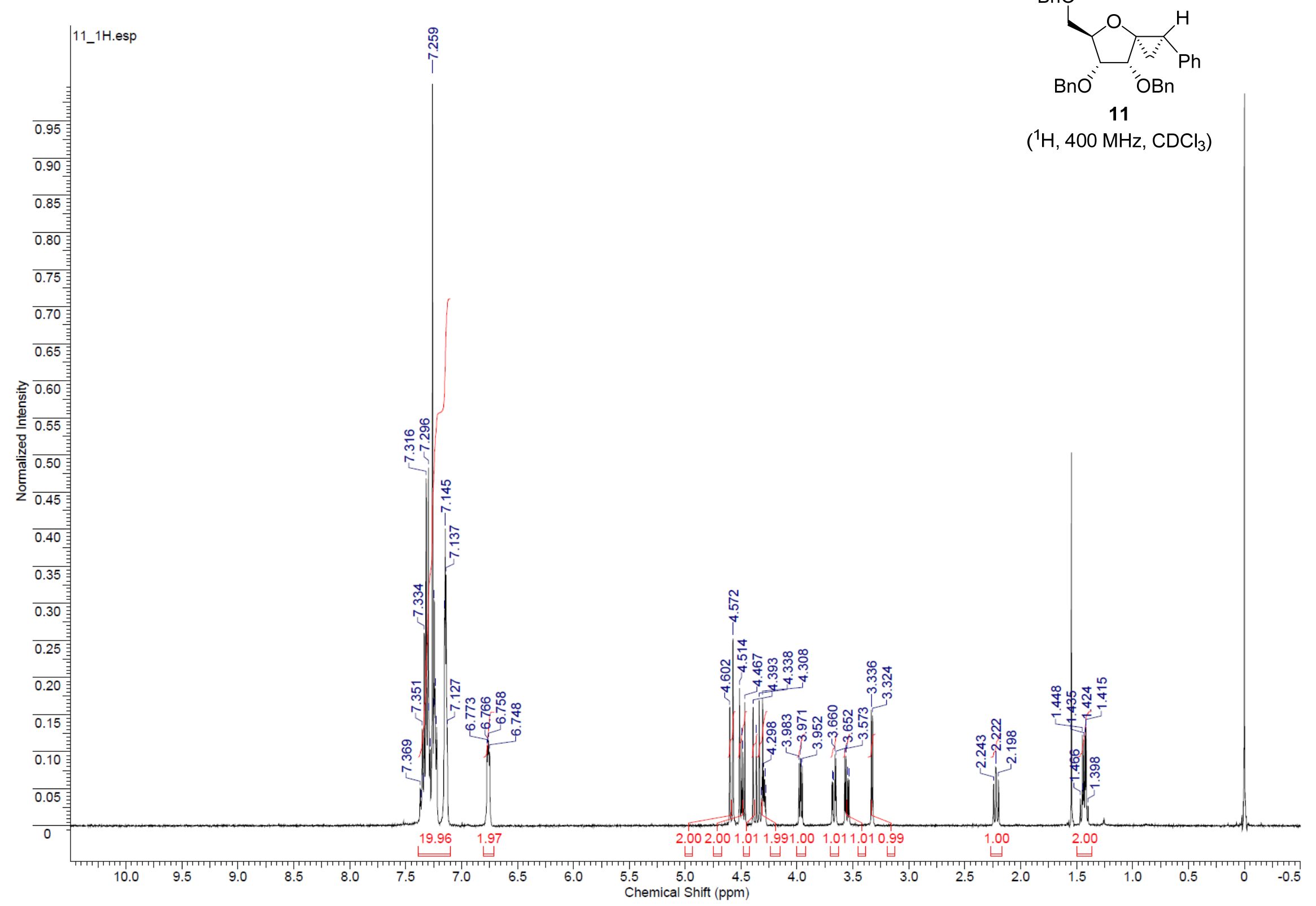




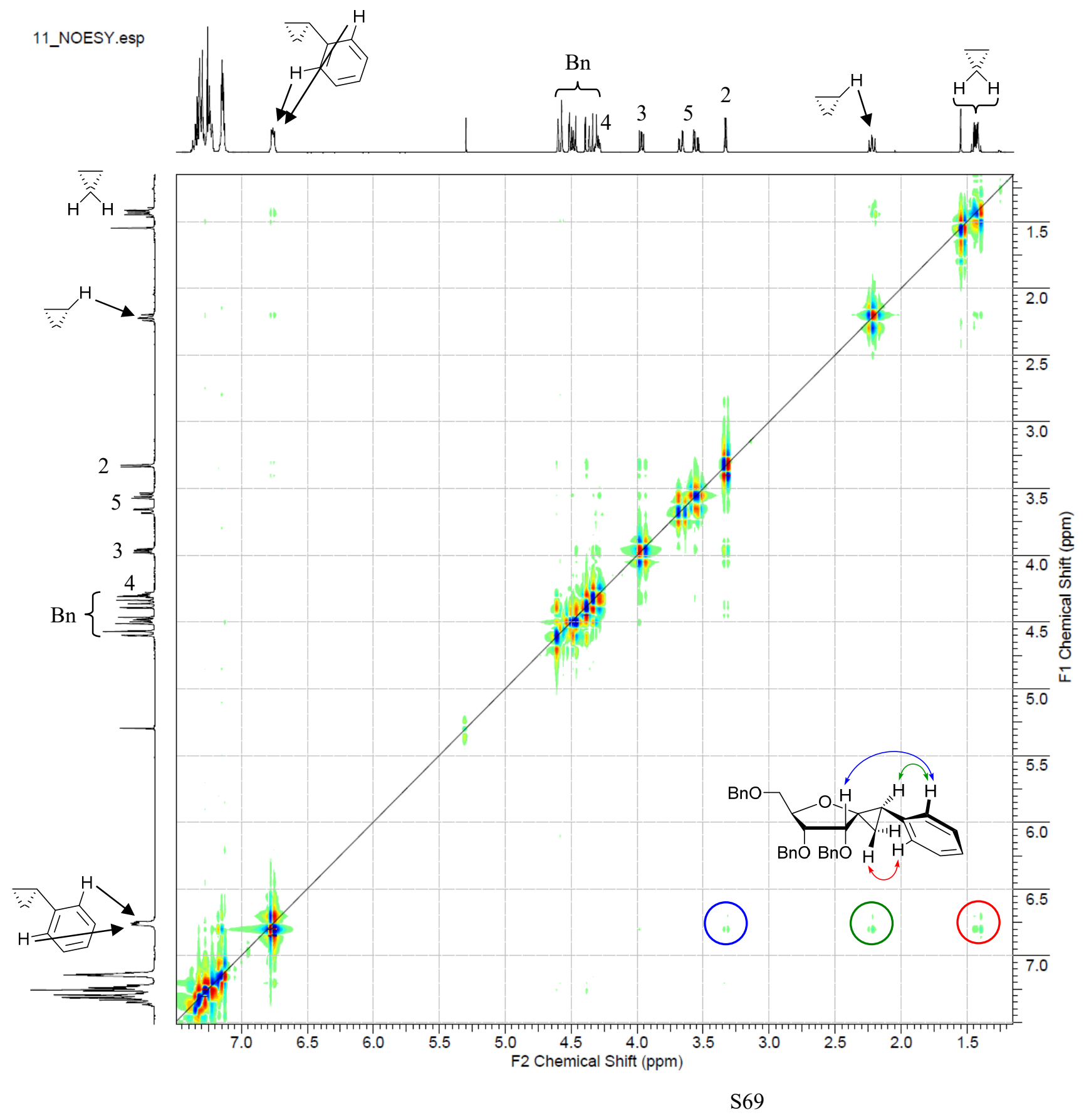




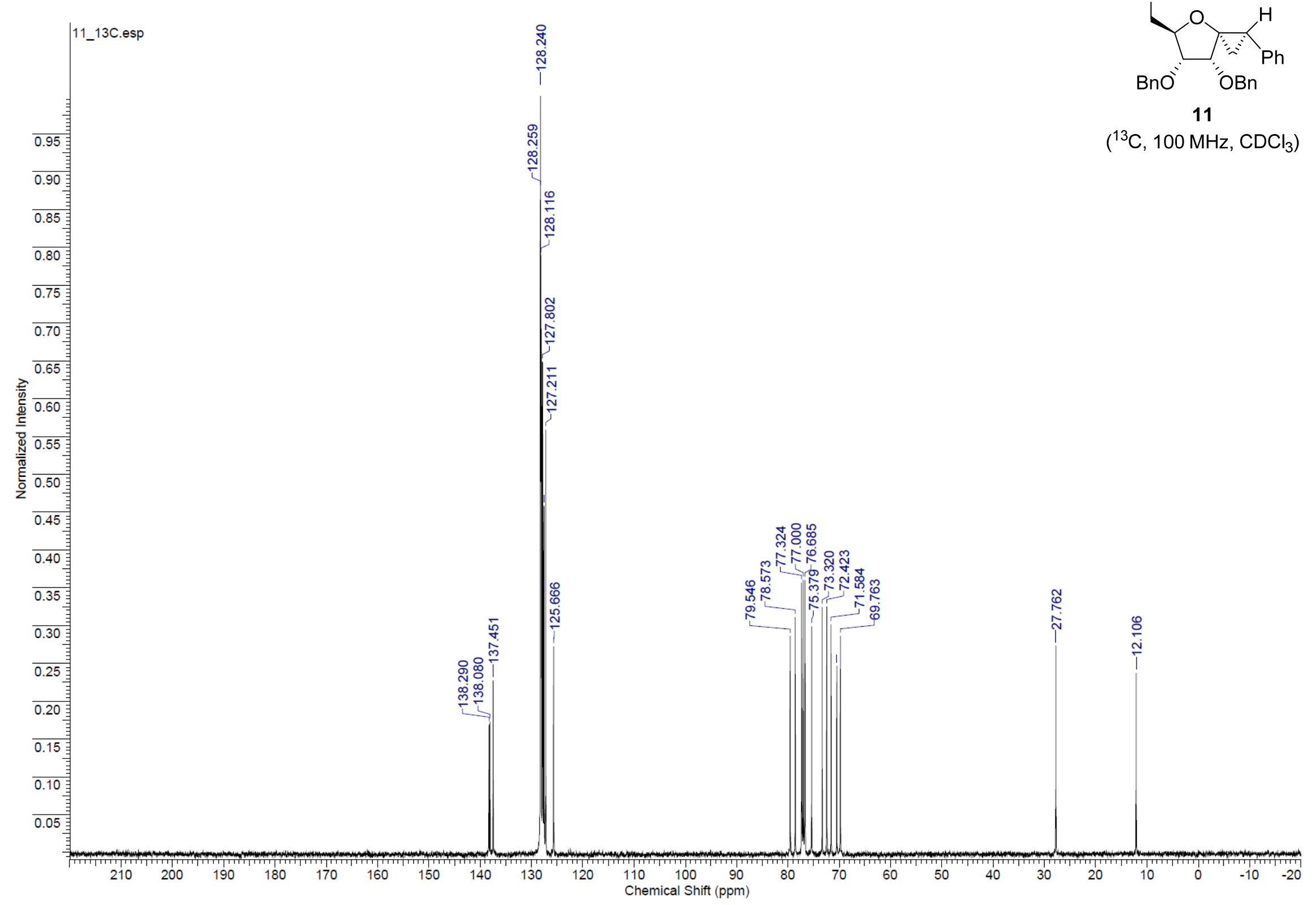




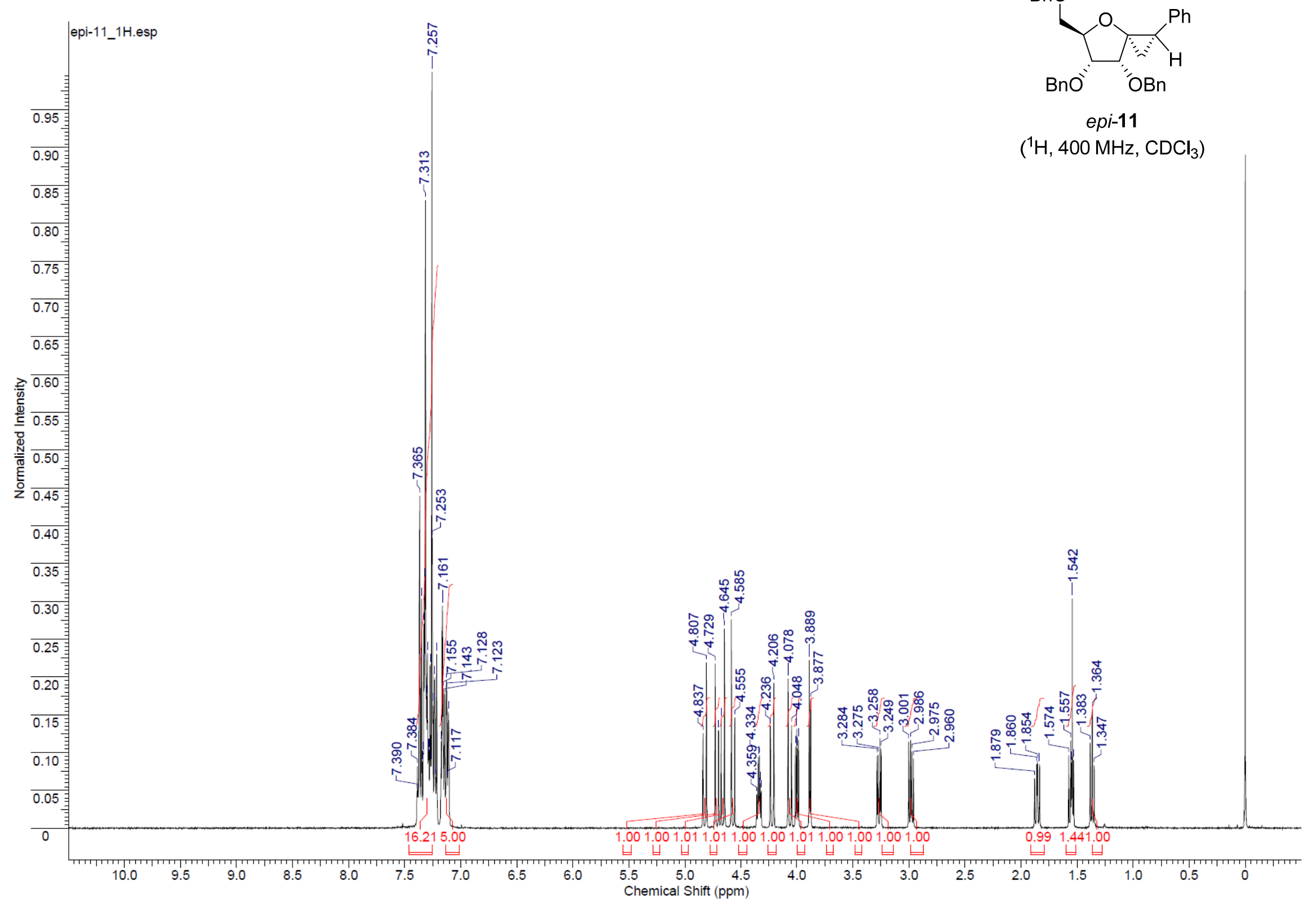



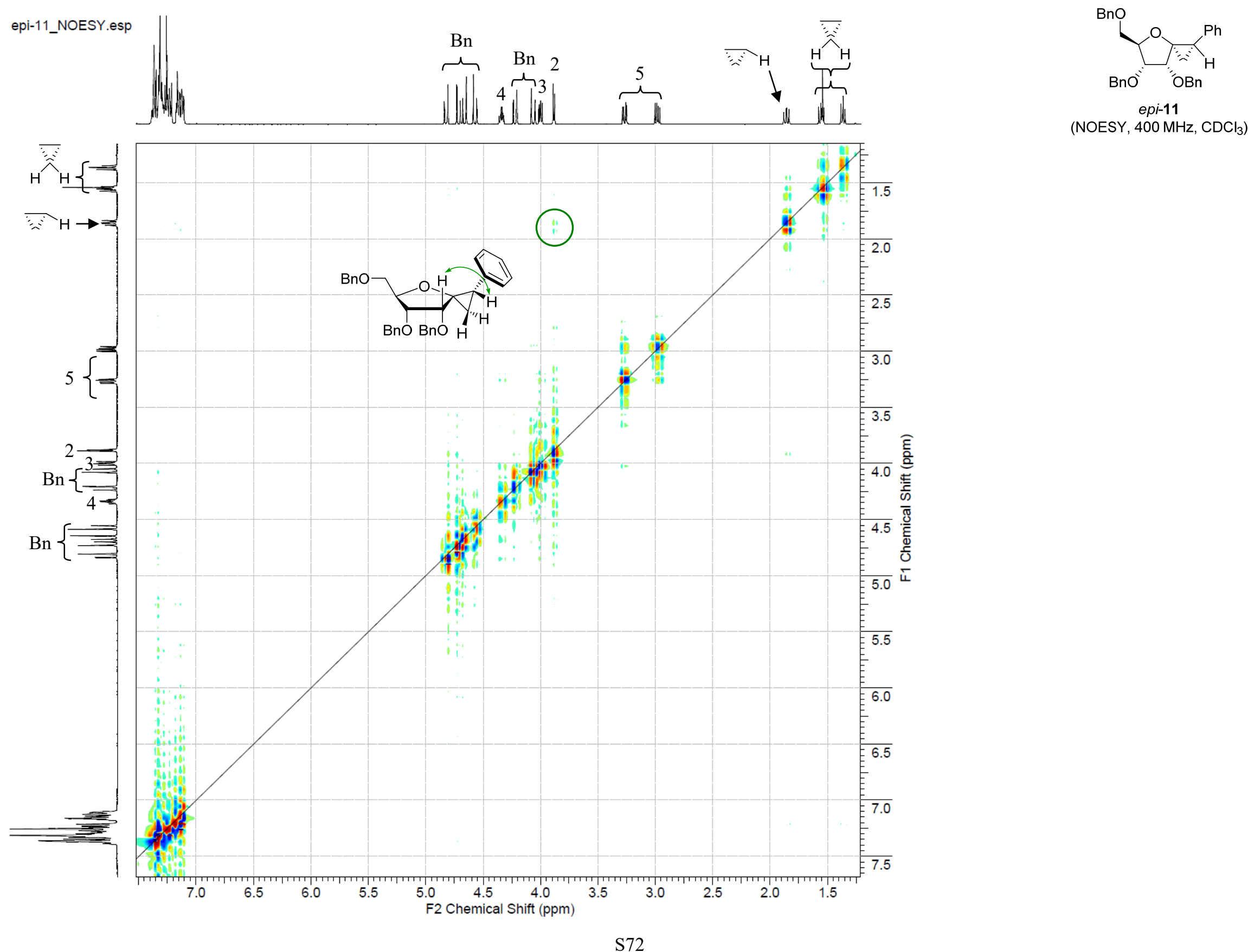


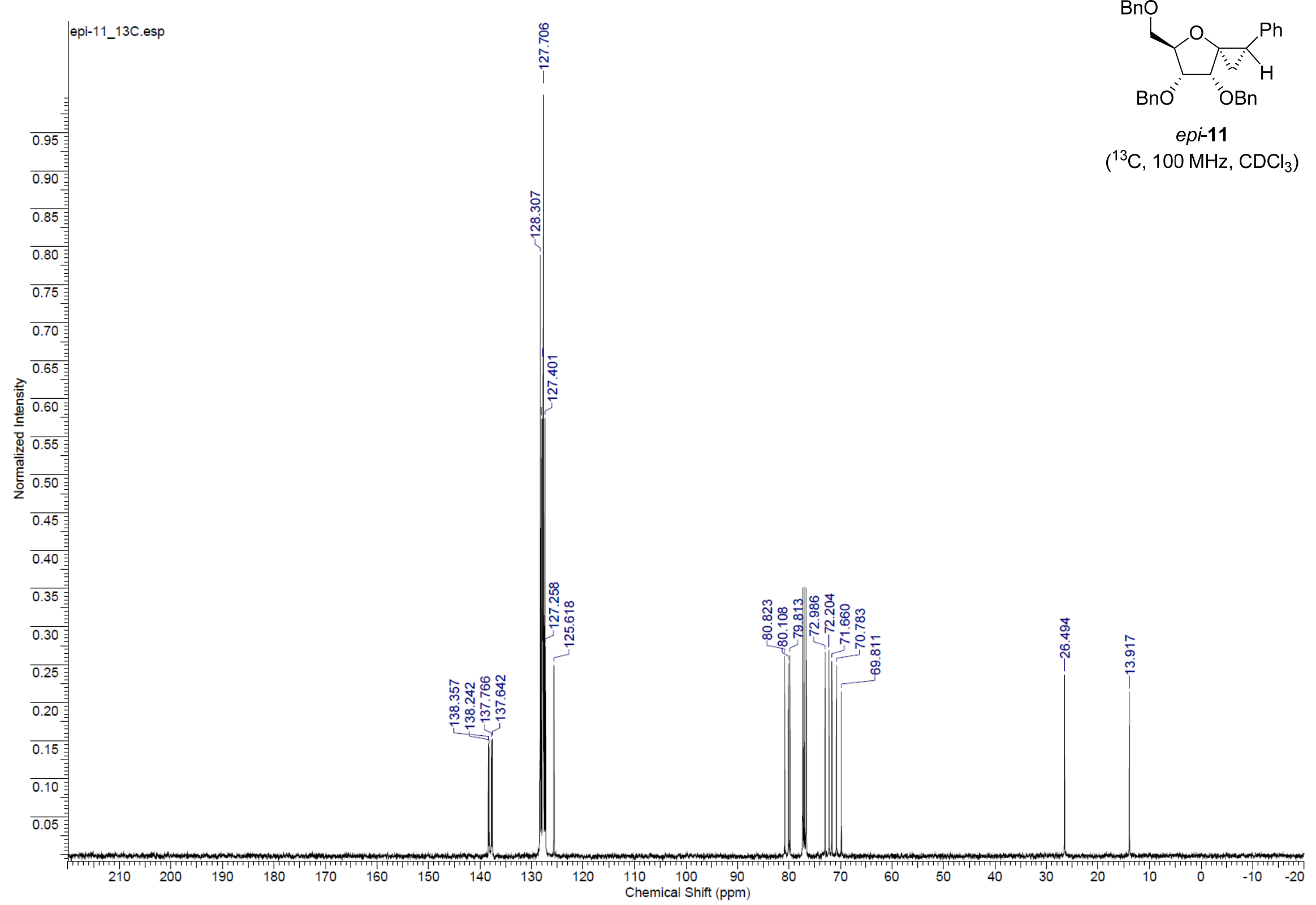




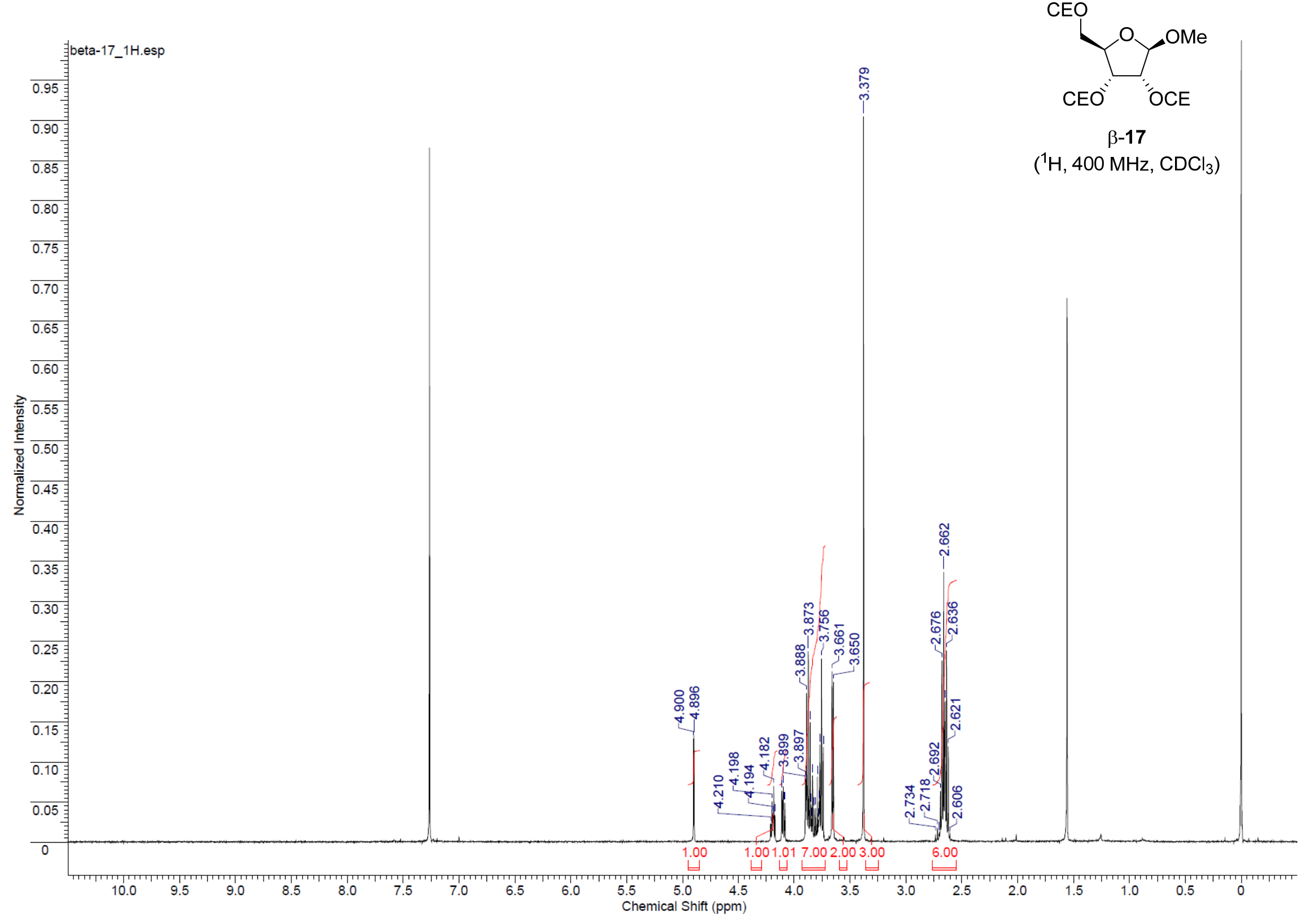




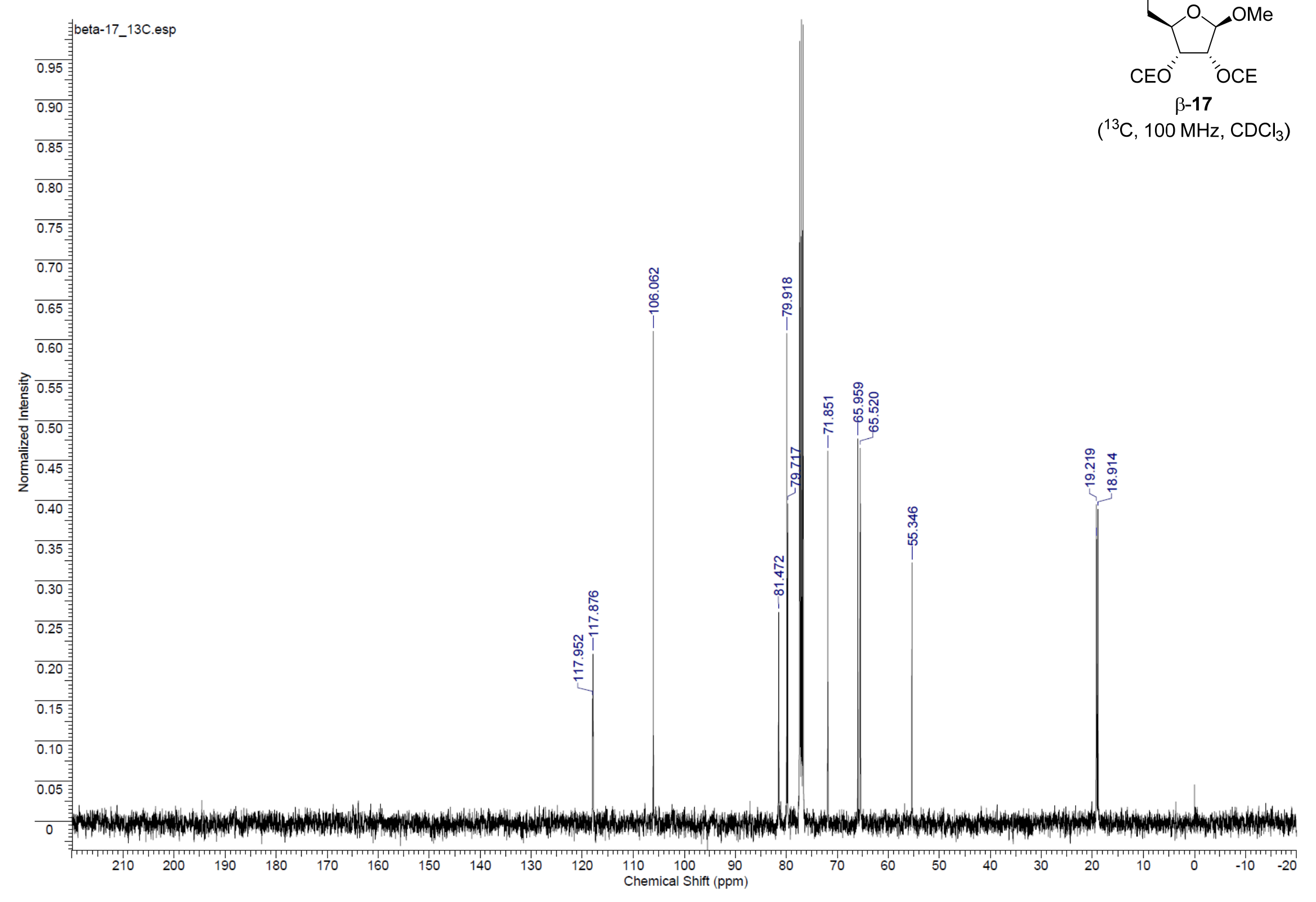




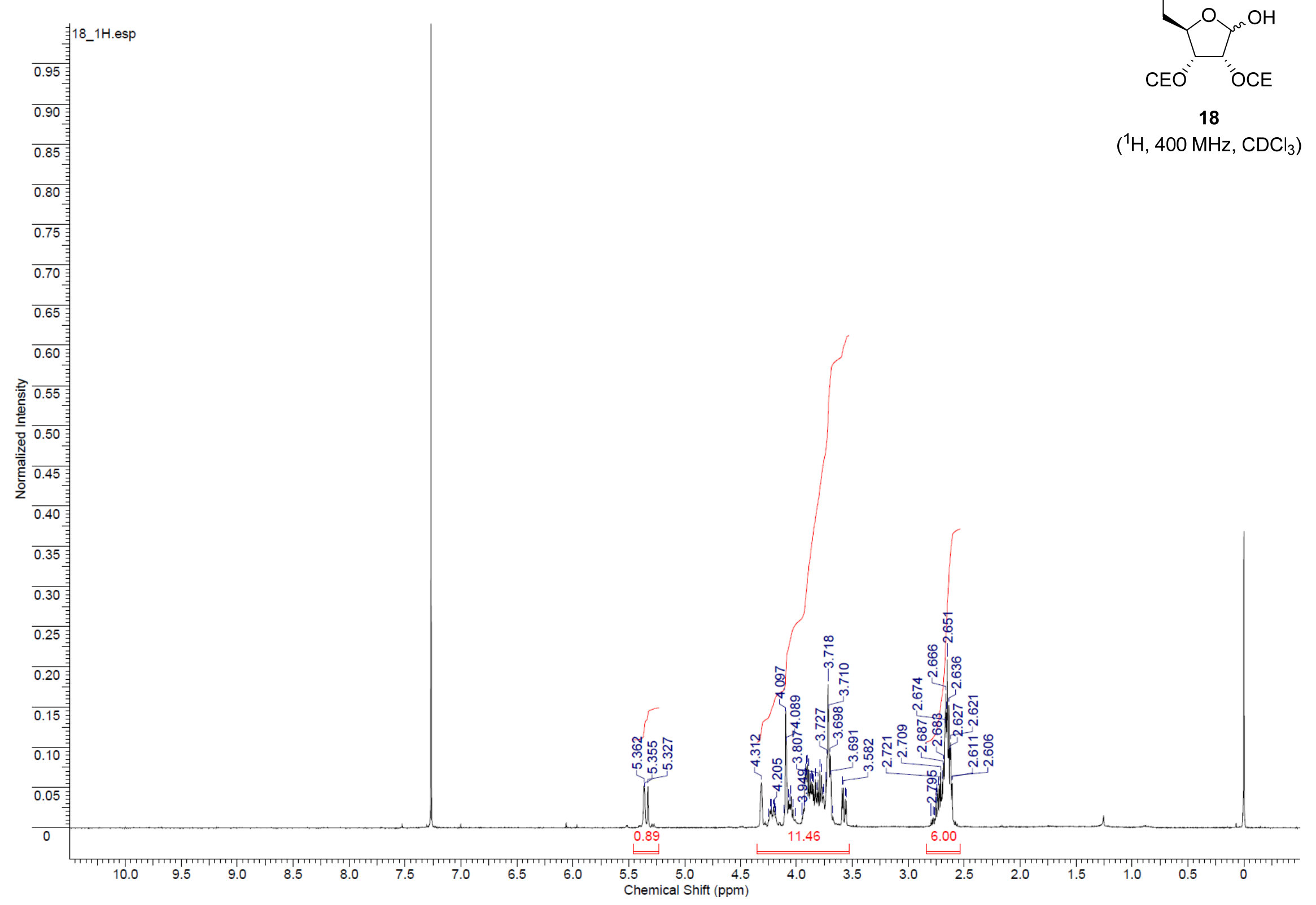




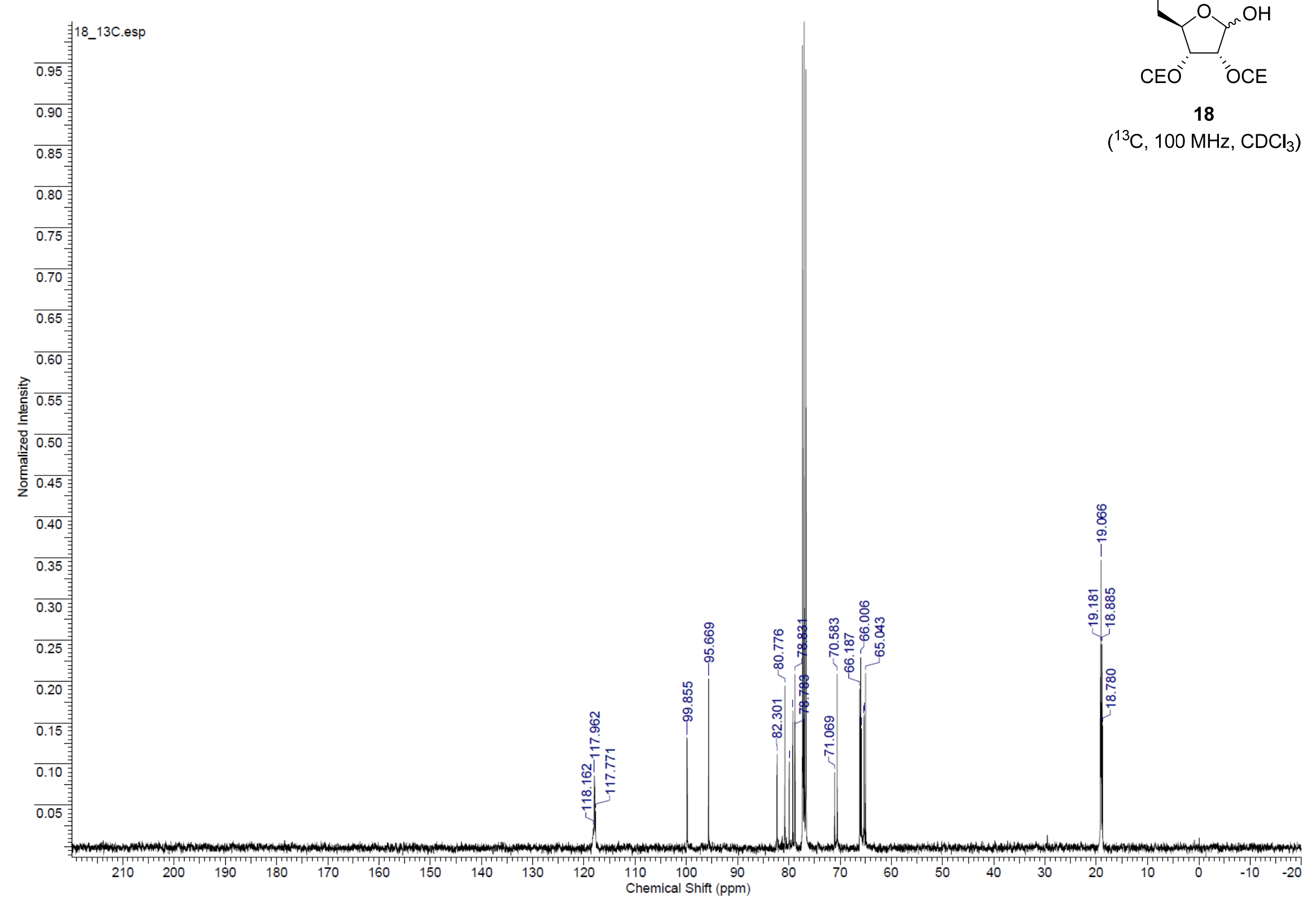




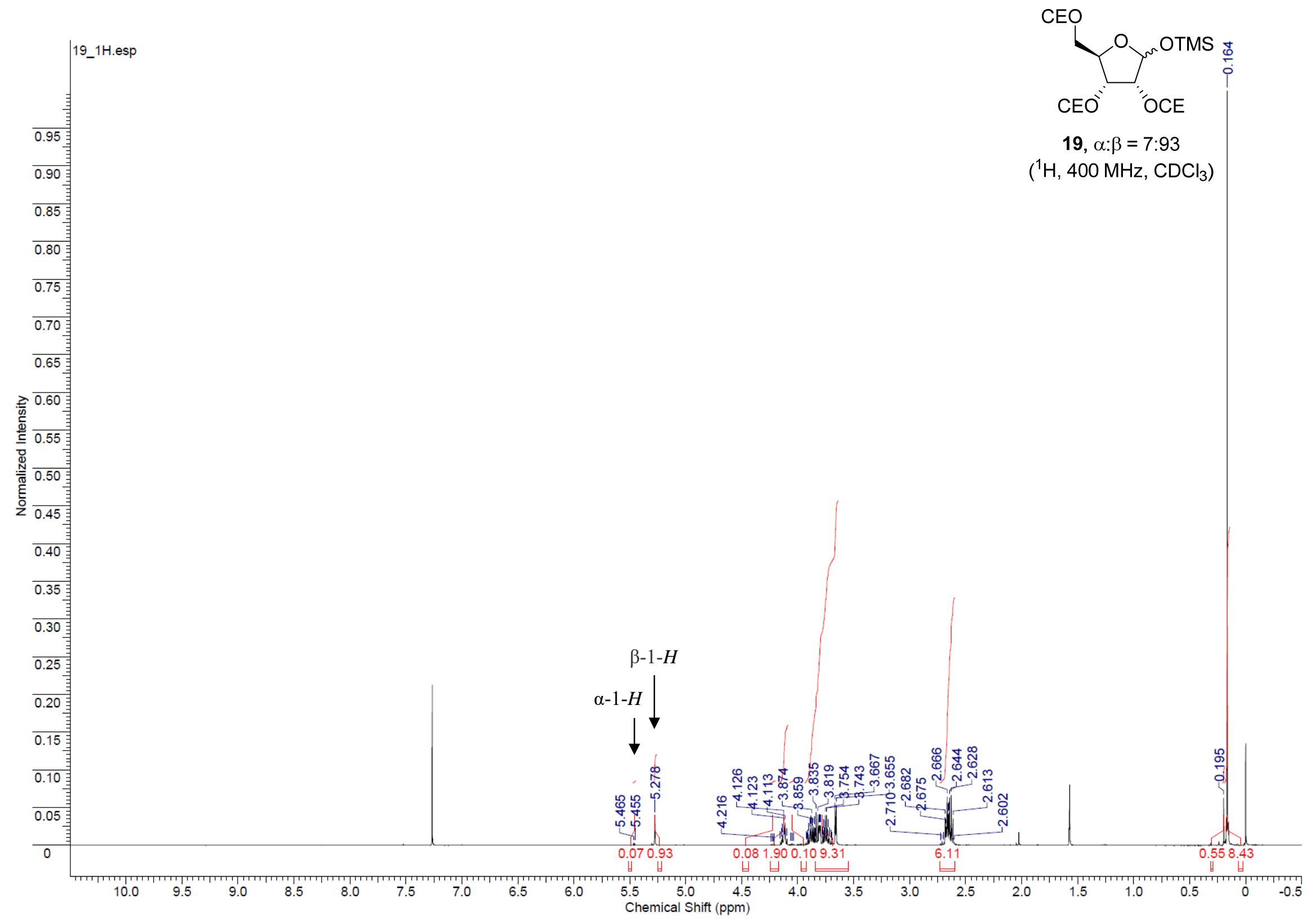




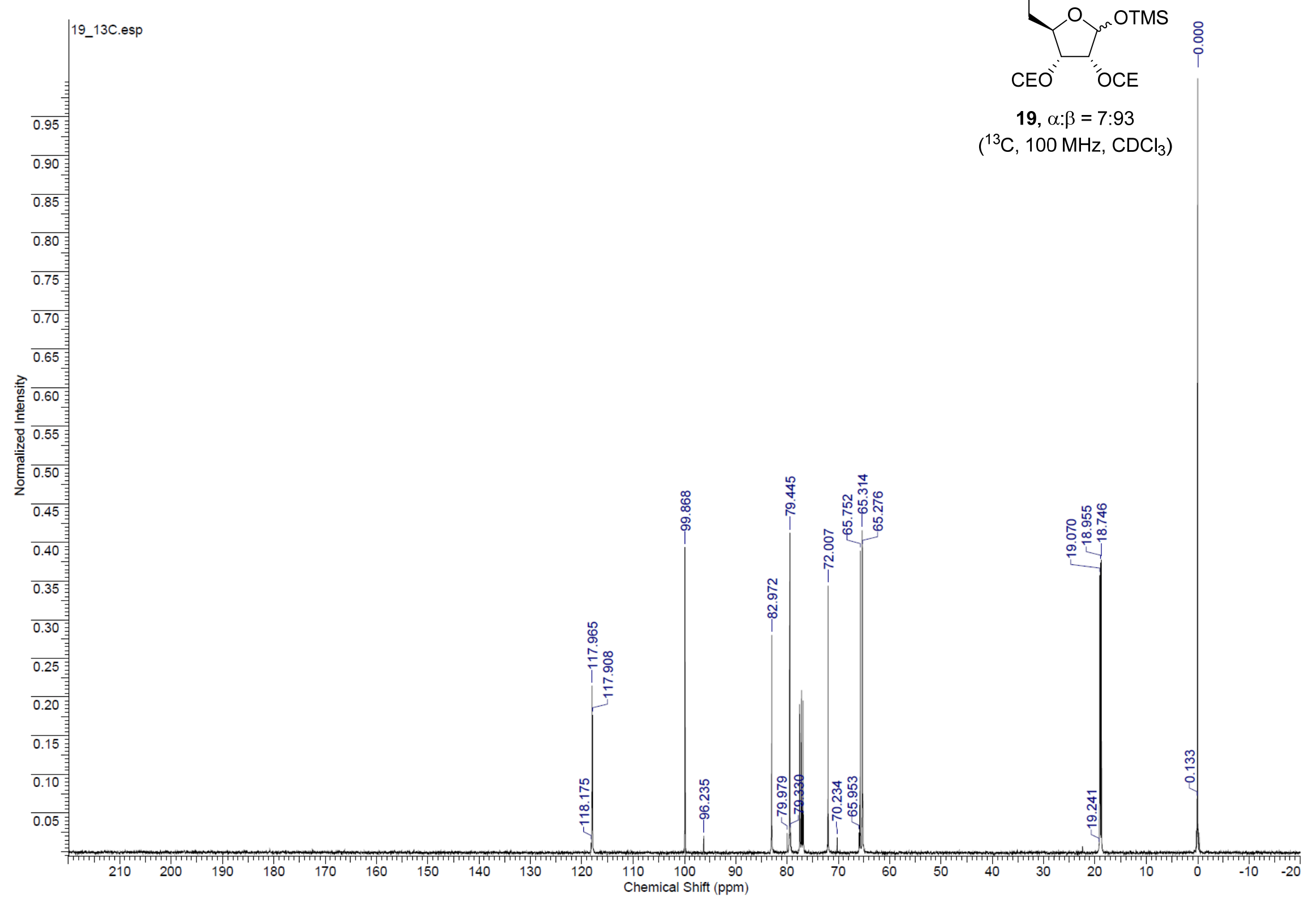




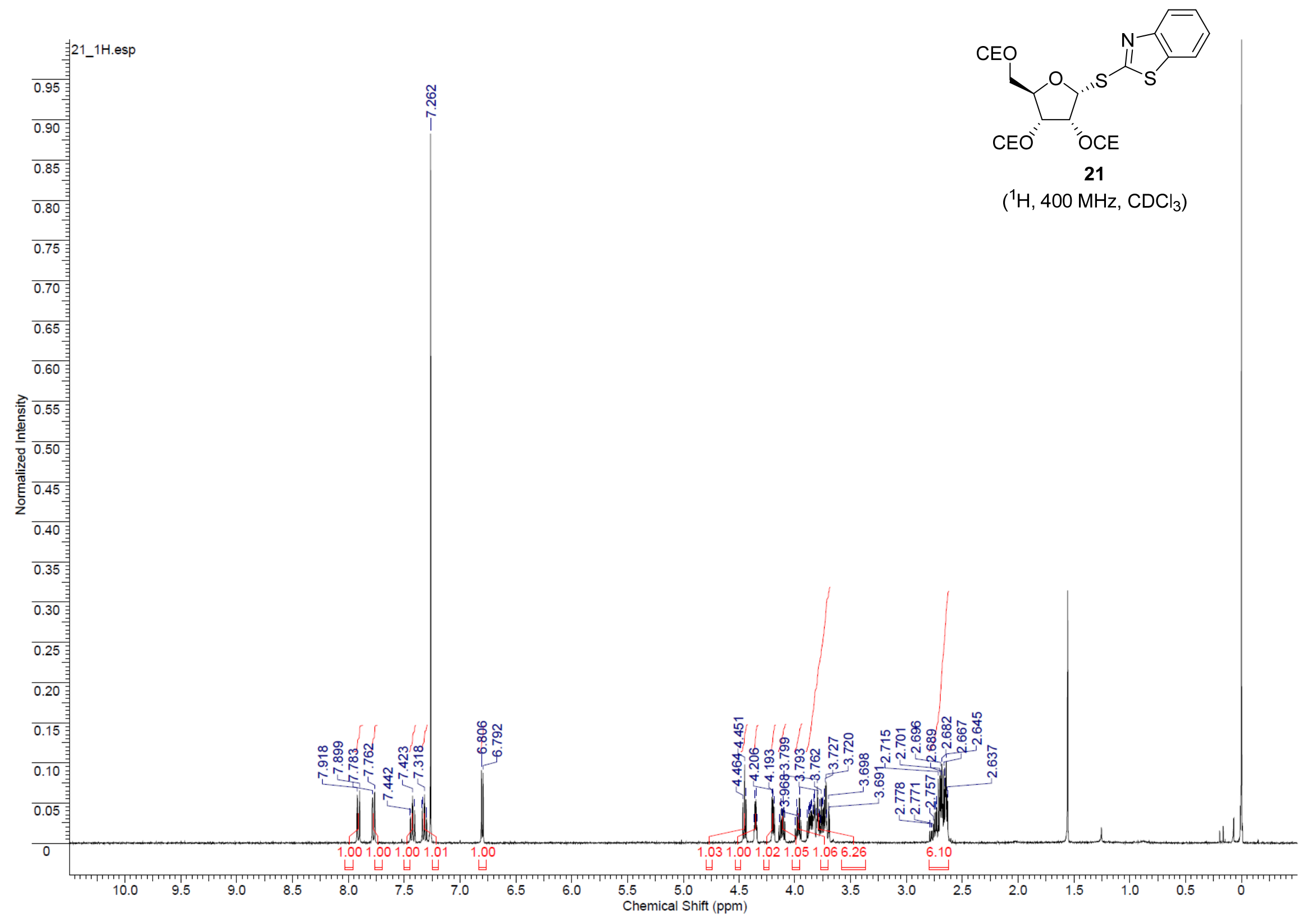




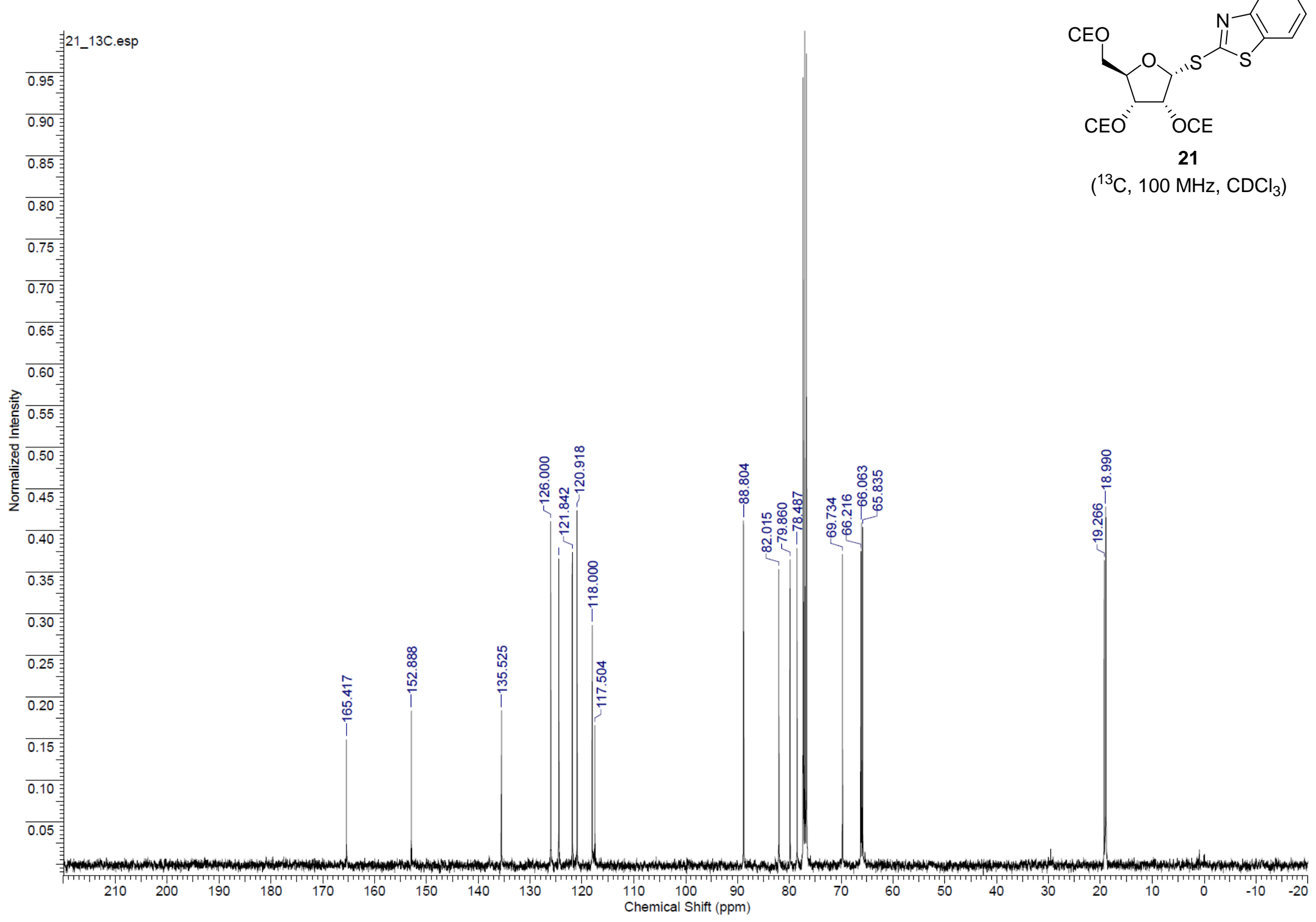




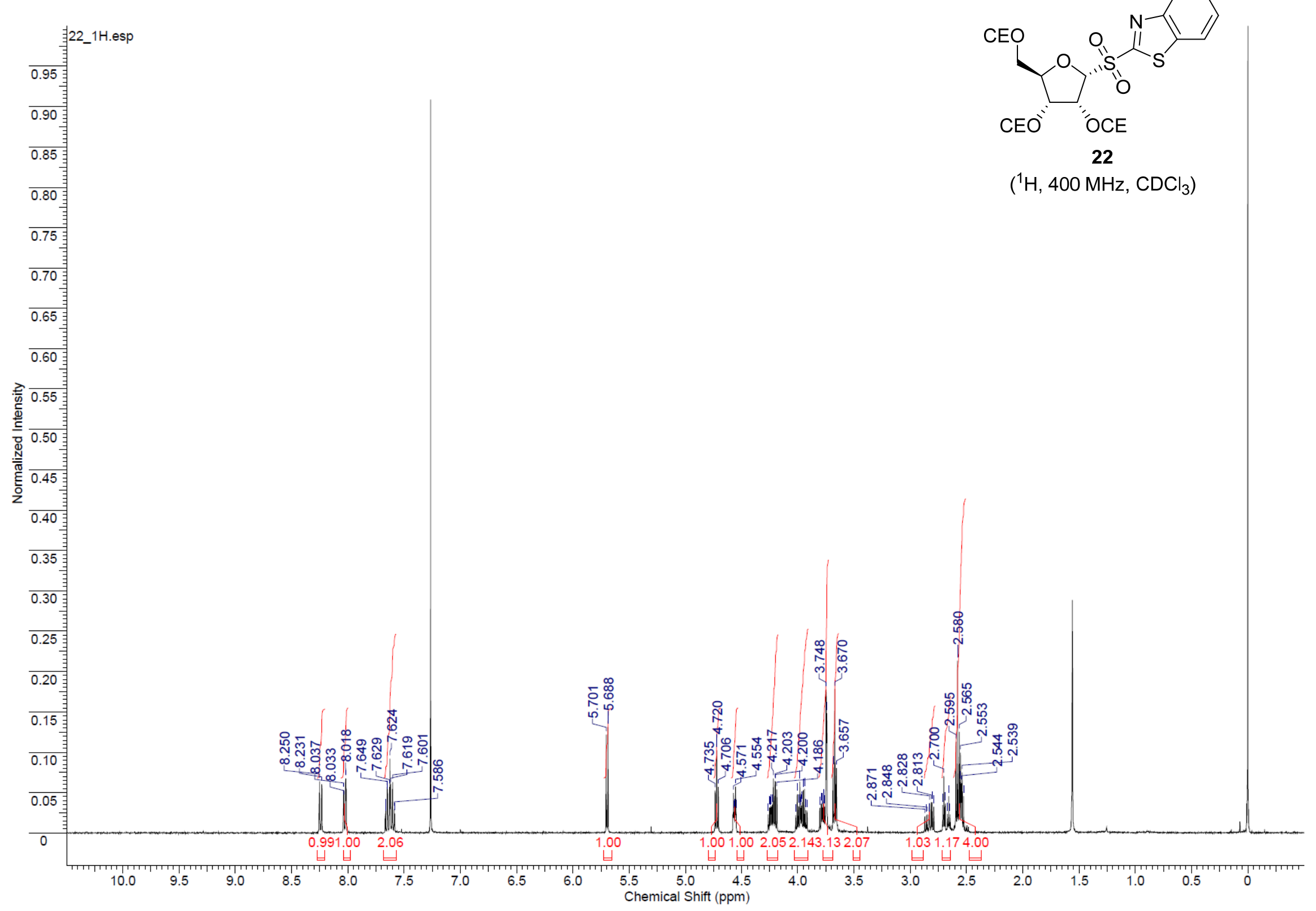




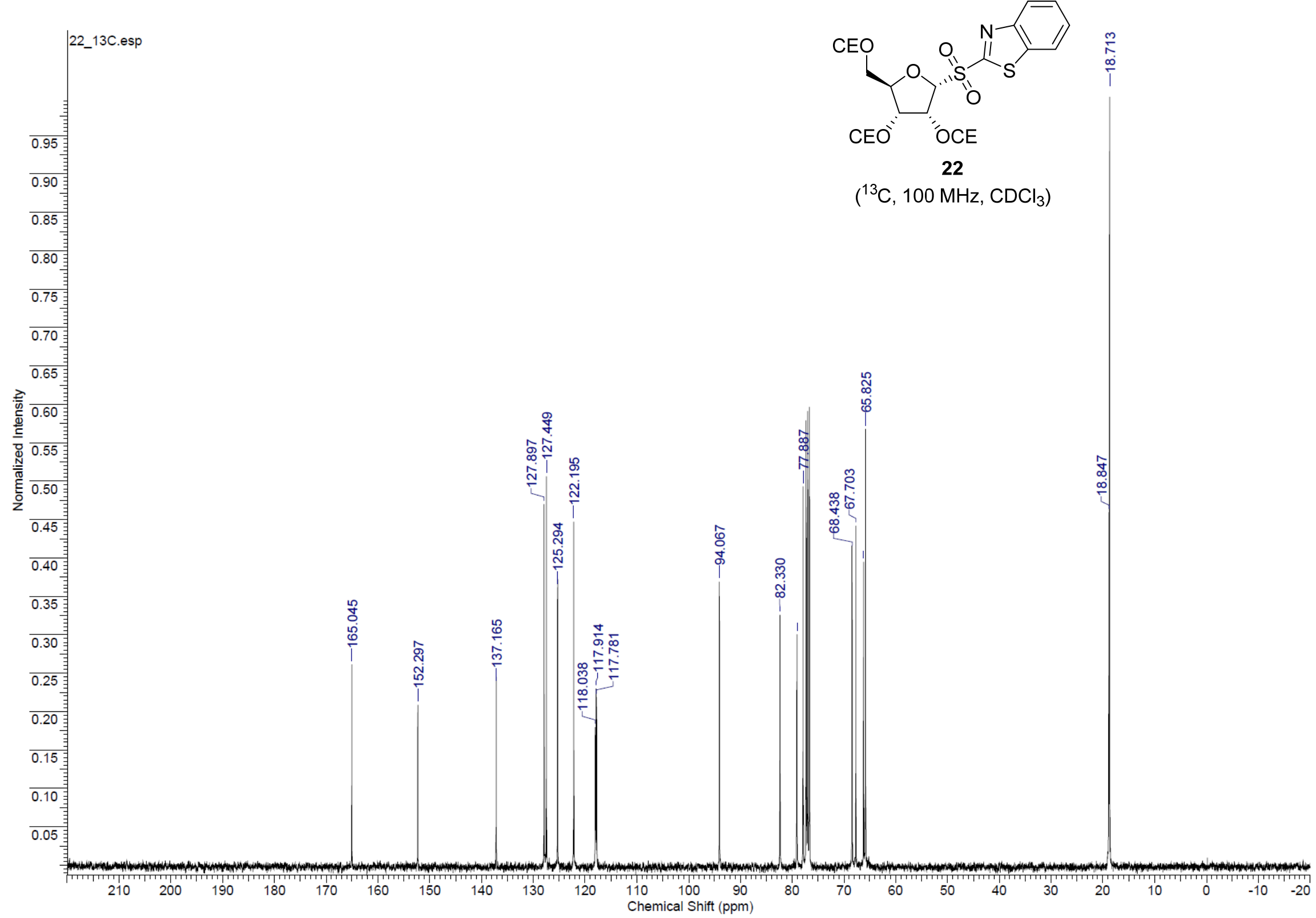




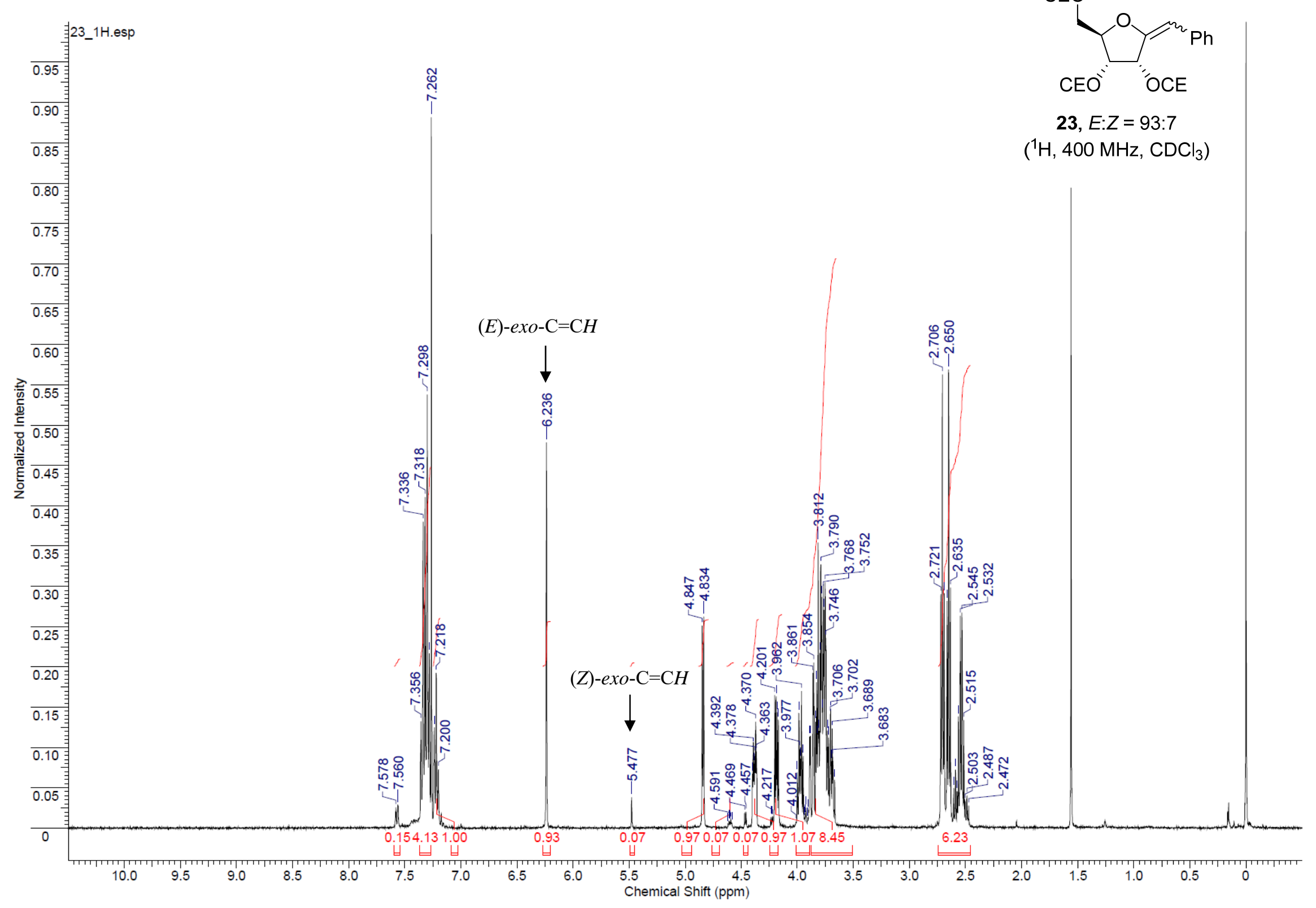




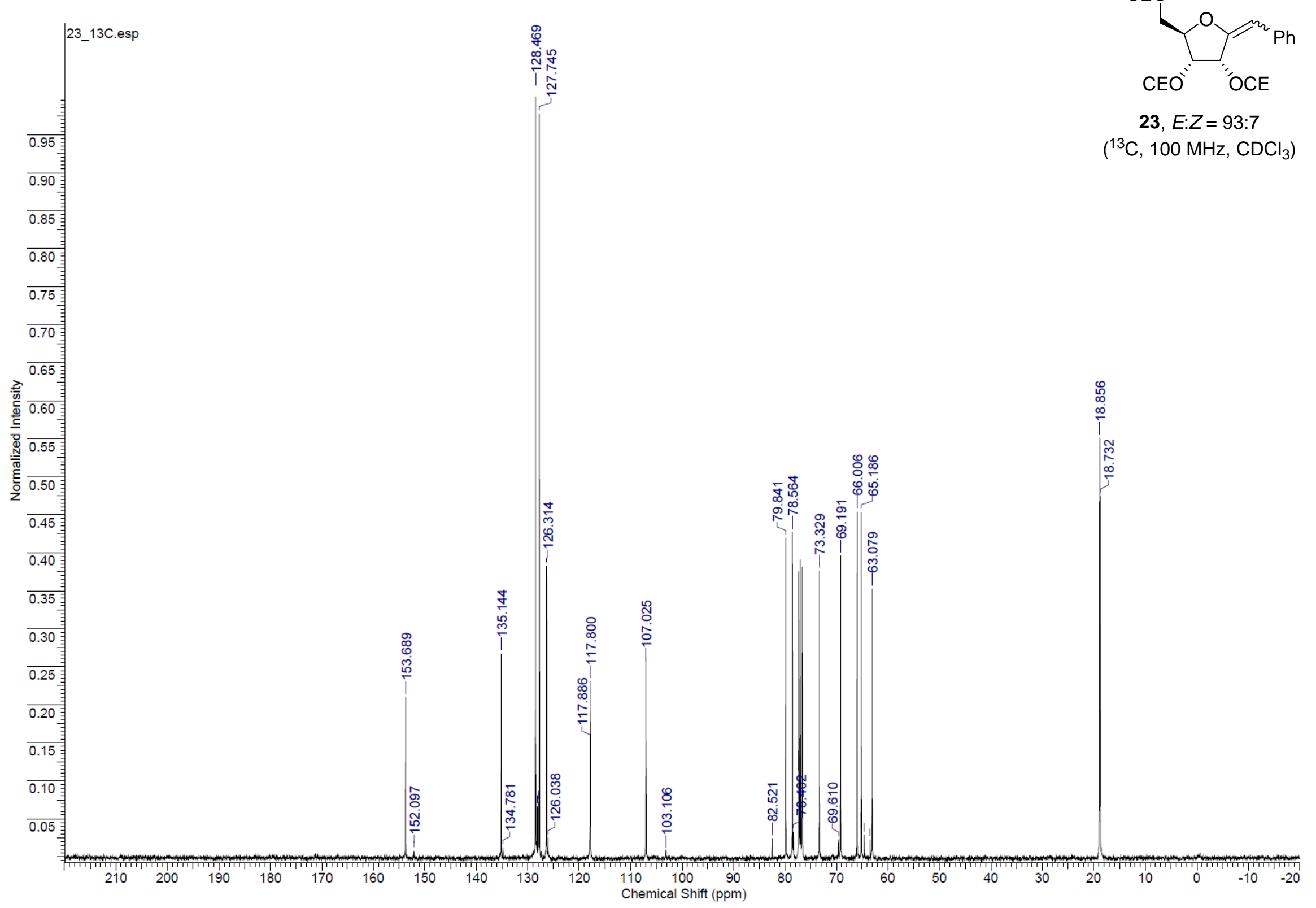




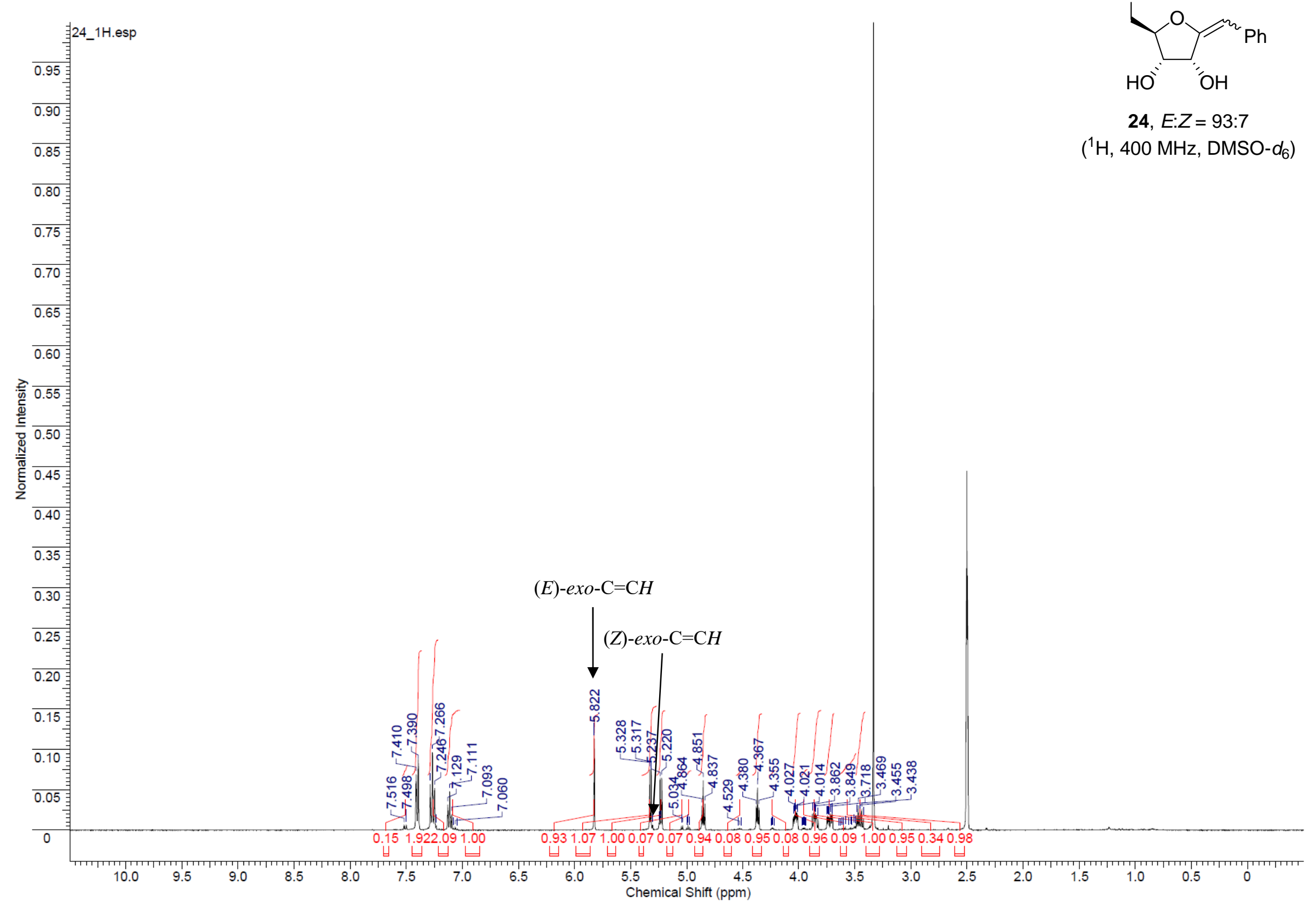




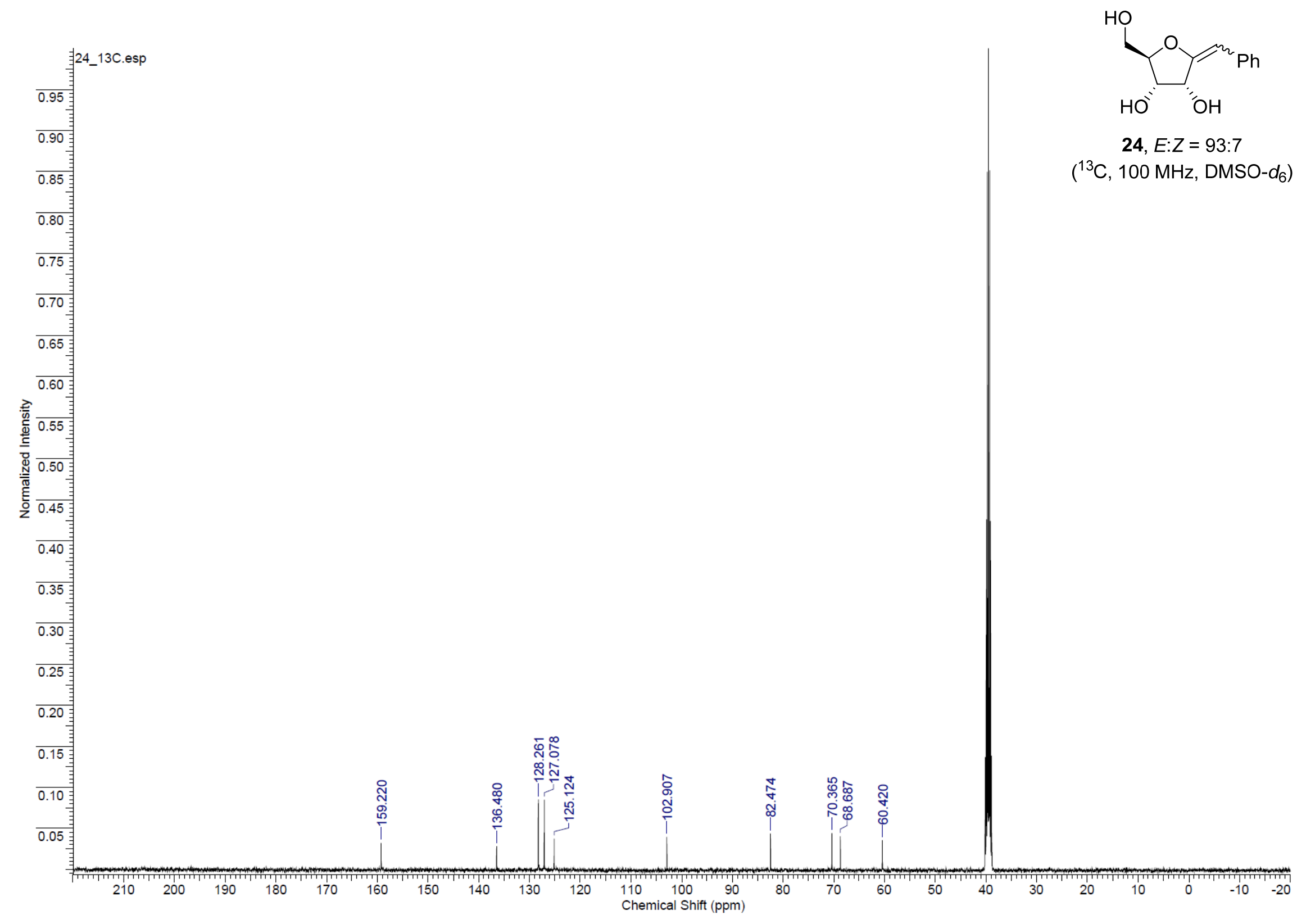

